\title{
DIFFUSION BONDING OF INCONEL 600 TO SILICON CARBIDE FOR NEXT
}

GENERATION HIGH TEMPERATURE APPLICATIONS

by

Yaiza Rodriguez Ortego

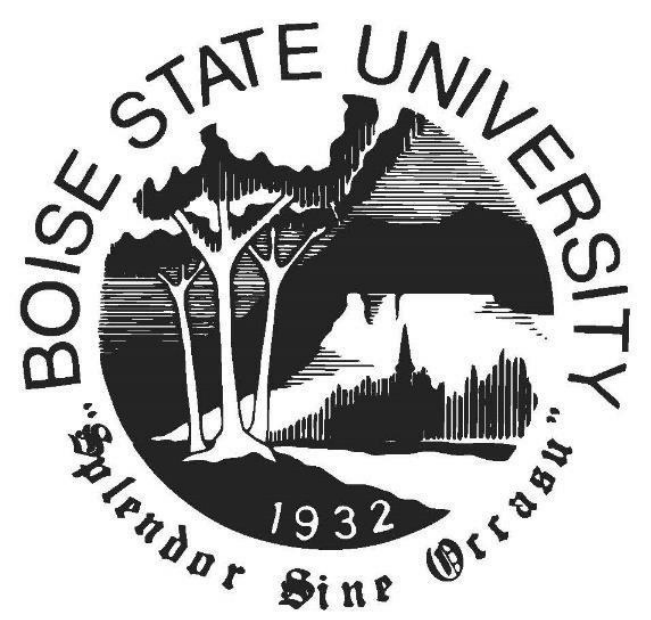

A thesis

submitted in partial fulfillment of

the requirements for the degree of

Master of Science in Materials Science and Engineering

Boise State University

May 2020 
(C) 2020

Yaiza Rodriguez Ortego

ALL RIGHTS RESERVED

BOISE STATE UNIVERSITY GRADUATE COLLEGE

\section{DEFENSE COMMITTEE AND FINAL READING APPROVALS}

of the thesis submitted by

Yaiza Rodriguez Ortego

Thesis Title: Diffusion Bonding of Inconel 600 to Silicon Carbide for Next Generation High Temperature Applications

Date of Final Oral Examination: $\quad 12$ March 2020

The following individuals read and discussed the thesis submitted by student Yaiza Rodriguez Ortego, and they evaluated their presentation and response to questions during the final oral examination. They found that the student passed the final oral examination.

Brian J. Jaques, Ph.D. Chair, Supervisory Committee

Mike Hurley, Ph.D. Member, Supervisory Committee

Amy J. Moll, Ph.D. Member, Supervisory Committee

The final reading approval of the thesis was granted by Brian J. Jaques, Ph.D., Chair of the Supervisory Committee. The thesis was approved by the Graduate College. 


\section{ACKNOWLEDGMENTS}

The investigation presented within this document represents all my effort and dedication towards the fulfillment of the degree of Master of Science in Materials Science. It is with sincere gratitude that I acknowledge my supervisor, Dr. Brian J. Jaques. Under his guidance I have grown in more ways than I thought possible. His dedication to my development as an engineer and a professional will guide me long after my time as his student. I will always be grateful.

I would also like to extend my deepest appreciation to Dr. Amy Moll, not only for accepting the invitation to be a member of my committee but for her daily encouraging conversations and dedication to help students like myself meet their goals. Additionally, I would like to express my sincere thanks to Dr. Mike Hurley for the time and support he given me as a committee member.

A million thank-yous to the staff and students of the Advanced Materials Laboratory: Allyssa Bateman, Sam Pedersen, Timothy Phero, Addie Lupercio, Jennifer Watkins, Jordan Vandegrift, Scott Riley, Sandeep Dhakal, Luke Schoensee, Riley Winters, Kaelee Novich, Caden Doyle, Sam Cutler, Beck Perrine, Bette Gougar, Shannon Crain, and Taylor Nowling. I never would have survived without your support, time, and friendships. 
Lastly, thank you to Boise State University and all the dedicated professors within the Micron School of Material Science and Engineering who have personally influenced me.

Part of this work has been funded by the US Department of Energy, under the SBIR Award Number DE-SC0015118. The purpose of which is to develop a novel ceramic/metal seal design for heat exchanger applications to account for the energy lost as waste heat that could be repurposed as useable energy. This grant partnered Boise State University with Hifunda Inc. This work has also been partially funded by my supervisor Dr. Brian J. Jaques, who generously funded me to complete a handful of experiments that would improve my work and support my story.

To my loving parents, Merche Ortego and Juan Domingo Rodriguez, thank you for always inspiring me and showing me that hard work pays off. Thank you to the friends who keep me up when life gets down: Brooke and Brittney Pahukoa, Marta Hermida, and Shay Shaw. Lastly, the Athletic Department at Boise State University and the lifelong mentors who have supported me from the very beginning of my journey here at Boise State: Gordy Presnell, Cody Butler, Carian Ramirez, Sunny Smallwood and Heather Sower.

\footnotetext{
ABSTRACT

Ceramic to metal interfaces are of interest for applications in extreme environments because they allow increased operational temperatures, resulting in greater thermodynamic efficiency in energy conversion processes. Ceramics offer high temperature corrosion resistance while metals offer robust and versatile solutions to assemblies. Understanding the solid-state reactions, the resulting interfacial
} 
microstructure, and the properties of the joints produced by diffusion bonding is essential for developing reliable ceramic to metal interfaces.

The combination of silicon carbide (SiC) and a nickel-based alloy (Inconel 600) offers improved strength and resistance to high temperature degradation. This work focuses on the understanding of the solid-state diffusion reactions at the interface between $\mathrm{SiC}$ and Inconel 600 using a Ag or Ag-Pd interlayer. The diffusion bonding experiments were performed with several process parameters, including temperature $\left(900-930{ }^{\circ} \mathrm{C}\right)$, uniaxial pressure (1-10 MPa), and bonding time (30-180 min). The effects of the process parameters on diffusion behavior and interfacial microstructure were investigated through scanning electron microscopy, energy dispersive X-ray spectroscopy, X-ray diffraction, and mechanical testing. In most cases, brittle interfacial solid-state reaction phases of $\mathrm{Ni}$, $\mathrm{Cr}$, and Pd-silicides were formed and affected the interfacial microstructure. The thickness of the reacted layer was used to determine that the diffusion mechanism for both interlayer systems followed parabolic kinetics, which is indicative that the process is diffusion controlled. The precise control of diffusion reactions through bonding parameters is necessary to ensure the integrity and performance of the diffusion-bonded SiC-Inconel 600 transitions. 


\section{TABLE OF CONTENTS}

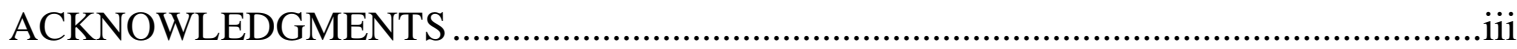

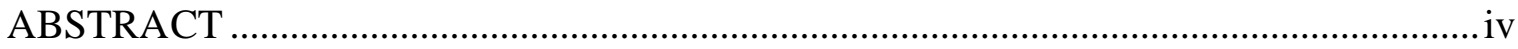

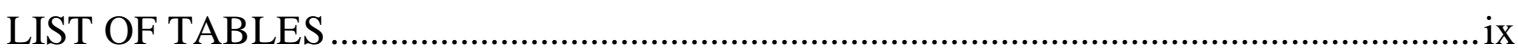

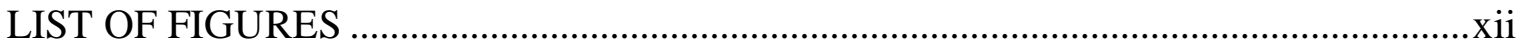

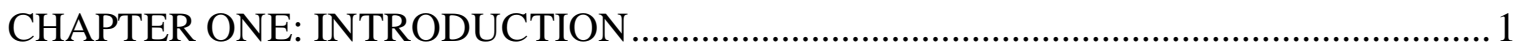

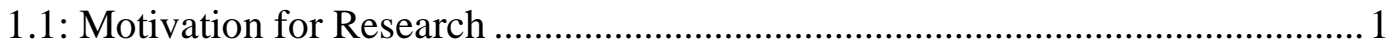

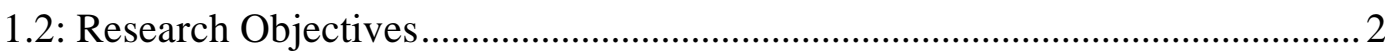

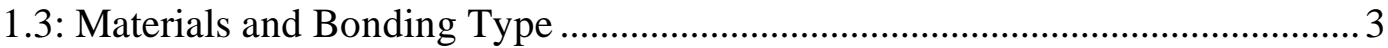

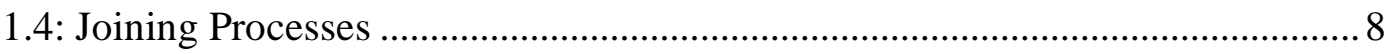

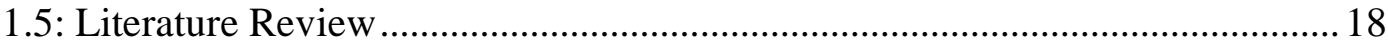

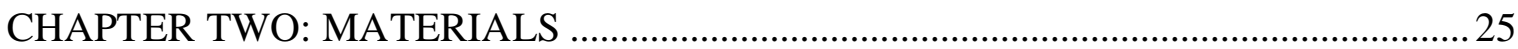

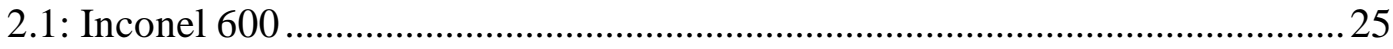

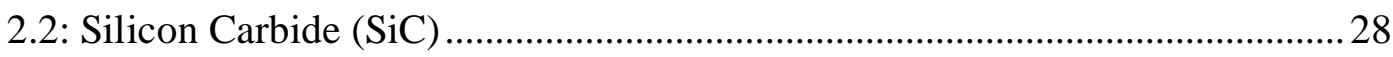

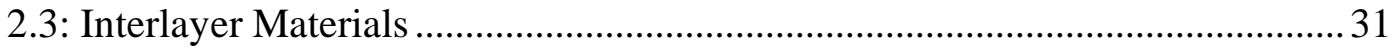

CHAPTER THREE: EXPERIMENTAL PROCEDURE ….............................................. 33

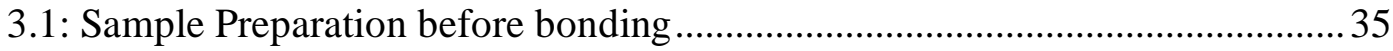

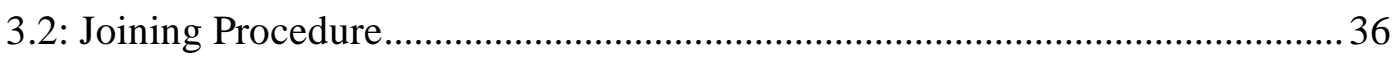

3.3: Sample Characterization ....................................................................... 42

3.3.1: Microstructural Characterization ..................................................... 42

3.3.2: X-Ray Diffractometry .......................................................... 43

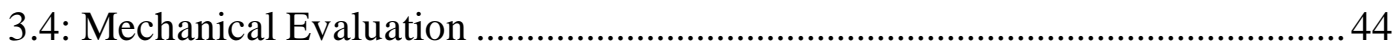

3.4.1: Tensile Test Evaluation.................................................................. 44 


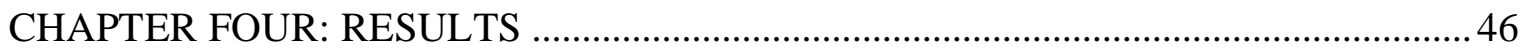

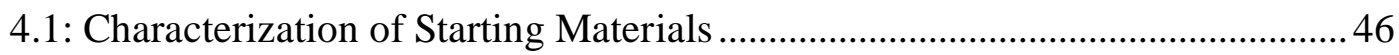

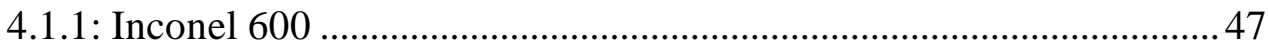

4.1.2: Reaction Bonded Silicon Carbide ( $\mathrm{SiC})$...................................... 49

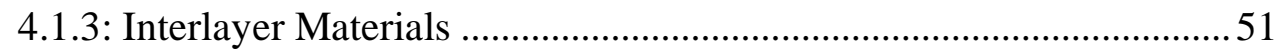

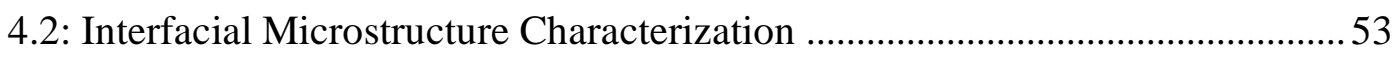

4.3: Comparison between the $\mathrm{Ag}$ and $\mathrm{Ag}_{75} \mathrm{Pd}_{25}$ interlayer systems .......................65

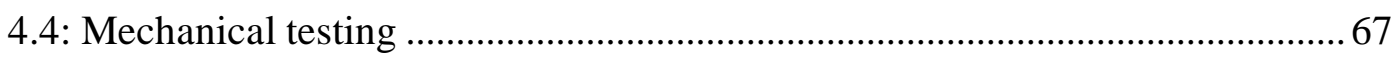

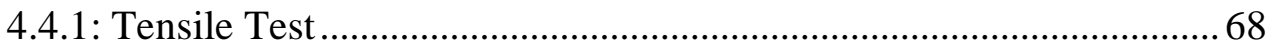

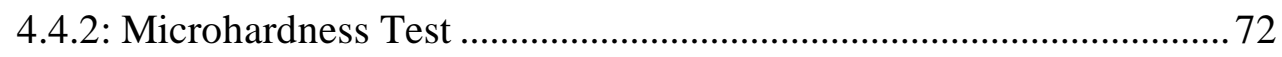

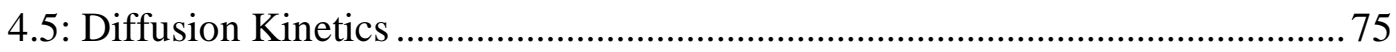

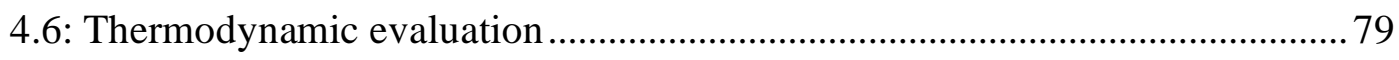

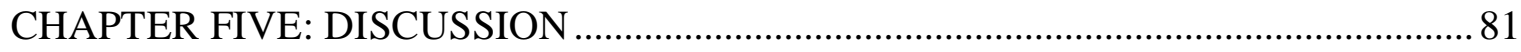

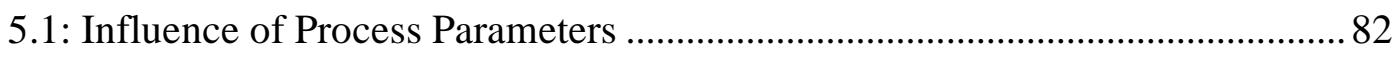

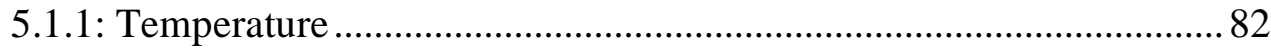

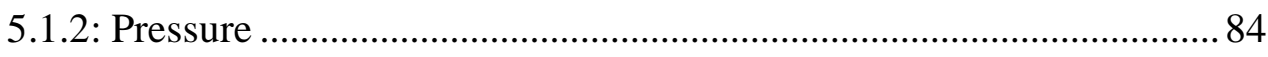

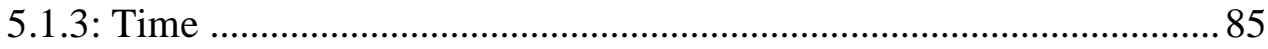

5.2: Effects of interlayer material on the interfacial microstructure ....................... 86

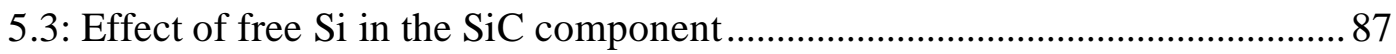

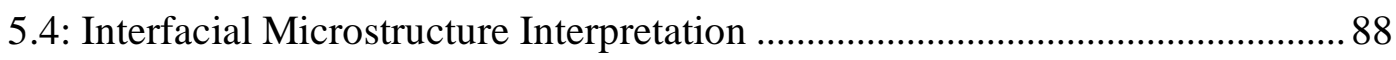

5.4.1: Microstructural Reactions on Ag interlayer system ........................ 92

5.4.2: Microstructure Results on the $\mathrm{Ag}_{75} \mathrm{Pd}_{25}$ interlayer system .............. 95 
5.4.3: Interface Thermal Cracking Analysis .......................................... 98

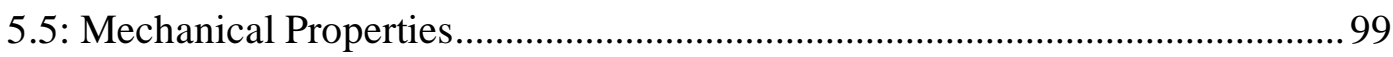

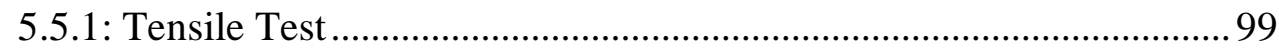

5.5.2: Microhardness Test ......................................................................... 101

5.6: Diffusion Kinetics ................................................................................... 101

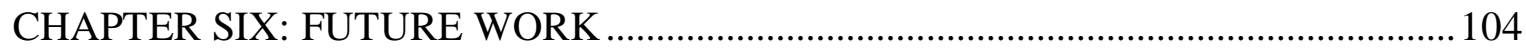

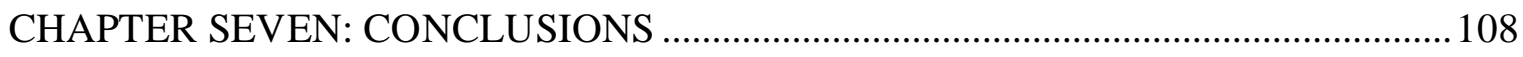

7.1: Conclusions on the Inconel 600/Ag/SiC Joints ............................................. 108

7.2: Conclusions on the Inconel $600 / \mathrm{Ag}_{75} \mathrm{Pd}_{25} / \mathrm{SiC}$ Joints ..................................... 109

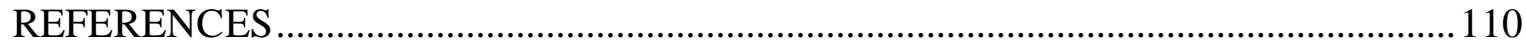




\section{LIST OF TABLES}

Table 1 Relevant literature on diffusion bonding dissimilar materials for complex

applications.

19

Table 2 The composition of the Inconel 600 superalloy used in this research study

as received from Special Metals.

Table 3 The Inconel 600 super alloy physical properties. All properties shown are from the CES EduPack database [26].

Table $4 \mathrm{SiC}$ characteristics. All properties shown are from the CES EduPack

database [26].

... 30

Table 5 Silver alloy characteristics. All properties are from the CES EduPack

database [26].

... 32

Table 6 Silver-Palladium 25\% alloying characteristics. Properties are measured

using the rule of mixtures as explained below [26]. 33

Table $7 \quad$ Process parameter matrix for samples ........................................................ 36

Table 8 Inconel 600 concentration in wt \% table from 10 EDS point scans on as-

received pellet. 
Table 9 Silver-Palladium $25 \%$ alloy concentration table from EDS point scans on

an as-received $75 \% \mathrm{Ag}-25 \% \mathrm{Pd}$

pellet. 54

Table 10 Elemental concentration from EDS point scans from Figure 34 for the

joint formed at $900{ }^{\circ} \mathrm{C}$ under $10 \mathrm{MPa}$ for 30

minutes with the Ag-

interlayer.

59

Table 11 EDS point scans from Figure 35 from reaction layers $\mathrm{A}$ and $\mathrm{B}$ representing

the joint formed at $930{ }^{\circ} \mathrm{C}$ under $10 \mathrm{MPa}$ for 180

minutes with an Ag-

interlayer.

60

Table 12 EDS point scans from the joint formed at 900

${ }^{\circ} \mathrm{C}$ under $1 \mathrm{MPa}$ for 30

minutes with a $75 \% \mathrm{Ag}-25 \% \mathrm{Pd}$

interlayer. 64

Table 13 EDS point scans from Figure 39 from the joint formed at $930{ }^{\circ} \mathrm{C}$ under 10

MPa for 180 minutes with a $75 \% \mathrm{Ag}-25 \% \mathrm{Pd}$

interlayer. 66

Table 14 The test matrix for kinetic studies for both joints formed with Ag and Ag$\operatorname{Pd} 25 \%$ interlayers. 76

Table 15 Diffusion measurements for the different formed joints presented through this study for joints formed with $\mathrm{Ag}$ as well as $\mathrm{Ag}-\mathrm{Pd} 25 \%$ interlayers. .. 78

Table 16 Screen calculations of diffusion coefficient from diffusion thickness measurements and bonding times from $\mathrm{Ag}$ interlayer system. 80 
Table 17 Enthalpy of formation of nickel silicides from the reaction of Ni and Si.

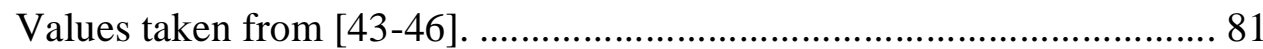

Table 18 Expected phases to possibly form at the Inconel 600/SiC interface of joint formed at $930{ }^{\circ} \mathrm{C}, 10 \mathrm{MPa}, 180 \mathrm{~min}$ (Figure 35), due to the diffusion of free $\mathrm{Si}$ into the metal component and the reaction of this $\mathrm{Si}$ with the base elements from the Inconel component such as $\mathrm{Ni}$ and $\mathrm{Cr}$, based on combined SEM/EDS/XRD/Literature data.

Table 19 Phases that formed at the Inconel 600/SiC interface of the joint formed at $900{ }^{\circ} \mathrm{C}, 1 \mathrm{MPa}, 30 \mathrm{~min}$ (Figure 37) ................................................ 99

Table 20 Phases that formed at the Inconel 600/SiC interface of the joint bonded at $930{ }^{\circ} \mathrm{C}, 10 \mathrm{MPa}, 180 \mathrm{~min}$ (Figure 39) 99 


\section{LIST OF \\ FIGURES}

Figure 1 Schematic illustration of metallic bonding showing the "sea of electrons" also known as the "electron cloud". Image modified from [18]. 5

Figure 2 Schematic representation of (a) the combination of $\mathrm{Na}^{+}$and $\mathrm{Cl}^{-}$ions forming $\mathrm{NaCl}$, which is a classic ionic bonding case [18]. 7

Figure 3

Schematic representation of covalent bonding in a molecule of methane $\left(\mathrm{CH}_{4}\right)$ illustrating the sharing of valance electrons between atoms [18]. ... 8

Figure 4

Schematic of the different possible joining techniques for ceramic/metal joints $[3,20]$.

Figure 5

(A) Illustration of chemical/physical bond by charge transfer across each component resulting in van der Waals bonding. (B) Liquid metal drop shape depending on contact time: (i) initial contact or solid surface contact and (ii) equilibrium arrangement on deformable solid surface. Image modified from $[21,22]$.

Figure 6

Diffusion bonding sequence illustration of ceramic/metal diffusion bonded joints with metallic interlayer. Image modified from [21]. 13

Figure 7

Diffusion couples used to understand the fundamental diffusion processes that can occur due to the materials present within the Inconel 600-AgSiSiC system.

Figure 8 Logarithm of D-vs-1000/T lines for the diffusion of $\mathrm{Cu}, \mathrm{Au}, \mathrm{Ag}$, and $\mathrm{Al}$ in Si [18]..... 15

Figure 9 CTE of the different materials used within this study as a function of Temperature [26, 27]. 16

Figure 10

Schematic illustration of thermal stress in joint interface and mode of cracking due to the difference of the thermal expansion coefficient upon cooling; (a) Edge cracks in ceramic and (b) Core cracks in ceramic. If (a) mode happens the CTE of the ceramic < the CTE of the metal and if (b) happens the CTE of the ceramic > the CTE of the metal. Figure adapted from [21].

Figure 11 Ashby plots showing how Inconel 600 compares to other commonly used high performance metals if plotting (a) maximum service temperature versus yield strength and (b) maximum service temperature versus tensile strength [26]. 
Figure 12 Ashby plot showing the comparison of all well suited metals for high temperature applications by plotting maximum service temperature versus price [26].

Figure 13

Ashby plots showing how SiC compares to other commonly used high temperature ceramics if plotting (a) maximum service temperature versus yield strength and (b) maximum service temperature versus tensile strength [26].

Figure 14

Ashby plot showing the comparison of all well suited high performance ceramics for high temperature applications by plotting their maximum service temperature versus price [26].

Figure 15

Schematic of the sample configuration with the silicon carbide ( $\mathrm{SiC}$ ) and Inconel 600 main components and the interlayer as $\mathrm{Ag}$ or $\mathrm{Ag}_{75} \mathrm{Pd}_{25} \ldots . . . .35$

Figure 16 Image (a) shows the FlexTest SE Plus coupled with the computer controlling the MTS. Image (b) shows the specific set up for Material Test System (MTS) mechanical test frame used for the formation of $\mathrm{SiC} /$ Inconel 600 joints with $\mathrm{Ag}$ and $\mathrm{Ag}_{75} \mathrm{Pd}_{25}$ as interlayers.

Figure 17 Experimental set up of the MTS mechanical test frame for diffusion bonding experiments showing a close up of a sample before bonding. .... 40

Figure 18

Schematic image showing the thermocouple set up to monitor the in-situ temperature during bonding experiments.

Figure 19

Diffusion bonding profiles showing temperature profile and applied pressure during formation of joint (a) at $900{ }^{\circ} \mathrm{C}$ for 30 minutes under 1 $\mathrm{MPa}$ and (b) at $930{ }^{\circ} \mathrm{C}$ for 180 minutes under $10 \mathrm{MPa}$. Green lines correspond to the actual measured temperature data. Blue lines correspond to the programmed pressure parameter.

Figure 20 Schematic sequence of the preparation of the formed joints to polish them and prepare for characterization.

Figure 21 Hitachi S-3400N-II Analytical Scanning Electron Microscope (SEM) for characterization from the Boise State Center for Materials Characterization at Boise State University [37].... 44

Figure 22 Rigaku Miniflex 600 bench-top X-ray diffractometer. The tool has a 600 $\mathrm{W}$ generator with copper source and is capable of a $2 \theta$ scan range of +2 to $+145^{\circ}$. It is capable of very quick scans because of the One-dimensional D/teX Ultra-high-speed detector. From the Boise State Center for Materials Characterization at Boise State University [38]. 45 
Figure 23

Tensile test fixture designed to conduct testing to investigate the bond strength for the formed SiC/Inconel 600 joints with $\mathrm{Ag}$ and $\mathrm{Ag}_{75} \mathrm{Pd}_{25}$ as the interlayer.

Figure 24

Schematic representation of a Vickers microhardness indent. Image (a) side view of how diamond pyramid indenter indents the surface of a sample and image (b) top view of indent mark on surface after indenting. Images adapted from Callister [18].....

Figure 25

XRD data of Inconel 600 as-received pellet. This pattern was used when characterizing the fracture surfaces of the Inconel 600 side. The shift observed is due to the slightly different lattice parameter of the Inconel 600 structure in comparison to the pure FCC structure due to the concentration of some $\mathrm{Fe}$ and $\mathrm{Cr}$ atoms within the structure. The presence of Fe and Cr within the Ni FCC structure do not change the crystal structure of the alloy, since Inconel 600 is a solid solution and the crystal structure remains unchanged from the structure of the base element, the lattice is affected by the difference in atomic radius.

Figure 26

XRD patterns of Inconel 600 showing the three expected peaks at $44^{\circ}, 51$ ${ }^{\circ}$, and $75^{\circ}$ from (a) study done by Y.C.S. Ravindra Kumar, et al. and (b) study done by W. Liu, et al. These Images were modified from [39, 40] and combined here.

Figure 27

Two SEM images of a cross section of a SiC rod received from Coorstek showing free $\mathrm{Si}$ in the $\mathrm{SiC}$ material. These images were used to calculate the amount of free $\mathrm{Si}$ present within the $\mathrm{SiC}$ component. 50

Figure 28

Typical Fiji processing software image showing the threshold colored image. The two different colors within the image correspond to two different phases and allow calculation of the $\%$ area of the red phase, which is the free Si phase.

Figure 29 XRD data of the $\mathrm{SiC}$ as-received pellet. The red squares described the $\mathrm{SiC}$ $(6 \mathrm{H})$ crystal structure peaks positions, the black squares represent the $\mathrm{Si}$ $(4 \mathrm{H})$ crystal structure peaks positions and the blue circles represent the $\mathrm{Si}$ peaks positions from the expected free $\mathrm{Si}$ present within the $\mathrm{Si}-\mathrm{SiC}$ matrix.

Figure $30 \quad$ XRD data of as-received Ag interlayer. 53

Figure $31 \quad \mathrm{XRD}$ data of the Ag-Pd 25\% alloy as-received interlayer. Some minor peaks are unknown, however the major peaks are indexed and represent a 80Ag-20Pd alloy phase, as well as some extra Pd phase.

\section{4}


Figure 32

Secondary electron SEM cross-section images showing the morphology at the interface of each bonded Inconel $600 / \mathrm{Ag} / \mathrm{SiC}$ joint: (A) $900{ }^{\circ} \mathrm{C}$ under 1 MPa for 30 minutes, (B) $900{ }^{\circ} \mathrm{C}$ under $10 \mathrm{MPa}$ for 30 minutes, (C) $930{ }^{\circ} \mathrm{C}$ under $10 \mathrm{MPa}$ for 30 minutes, (D) $930{ }^{\circ} \mathrm{C}$ under $10 \mathrm{MPa}$ for 180 minutes.

Figure 33

Two secondary electron SEM cross-section images showing joints formed at $900{ }^{\circ} \mathrm{C}$ under $1 \mathrm{MPa}(50 \mathrm{~N})$ for 30 minutes with $\mathrm{Ag}$ as the interlayer image (i) showing no diffusion but plastic deformation of the interlayer material and image (ii) showing a small diffusion as well as a small crack. SEM cross-section images, (a) shows the metal-metal interface and (b) shows the metal-ceramic interface of image (1).

Figure 34

Higher magnification of Figure 32 (B) showing the BSE SEM image where different reaction layers are observed. These regions observed within the reaction region show cracking within them. Compositions at each is collected and given in Table 10.

Figure 35

EDS map scans showing chemical concentration gradients of the elements of joint shown in Figure 32 (C). 60

Figure 36

Secondary electron SEM cross-section images showing the morphology at the interface of each bonded Inconel 600/AgPd/SiC joint fabricated at different combinations of process parameters, shown in Table 7. The magnifications used for each image is different to show all the different distinct regions within each of the joints.

Figure 37 Secondary electron SEM cross-section image, EDS map scans and EDS line scan of the joint formed at $900{ }^{\circ} \mathrm{C}$ under $1 \mathrm{MPa}$ for 30 minutes with $75 \% \mathrm{Ag}-25 \% \mathrm{Pd}$ interlayer shown in Figure 36 (A). This SEM images corresponds to the white box shown in Figure 36(A) at a higher magnification to take further observations.

Figure 38 Secondary electron cross-section SEM image of joint formed at $900{ }^{\circ} \mathrm{C}$ under $1 \mathrm{MPa}$ for 30 minutes with $75 \% \mathrm{Ag}-25 \% \mathrm{Pd}$ interlayer showing EDS point scans collected representing the different regions within the reaction layer that are observed within the Inconel 600 side.

64

Figure 39 Secondary electron cross-section SEM image of joint formed at $930{ }^{\circ} \mathrm{C}$ under $10 \mathrm{MPa}$ for 180 minutes. EDS map scans showing the different areas with the concentration gradient of elements. This SEM image corresponds to the white box shown in Figure 36 (C).

65 
Figure 40 Secondary electron SEM cross-section images of (a) Inconel 600/Ag/SiC joint formed at $900{ }^{\circ} \mathrm{C}$ under $1 \mathrm{MPa}$ for $30 \mathrm{~min}$, (b) Inconel 600/75\% $\mathrm{Ag}$ $25 \% \mathrm{Pd} / \mathrm{SiC}$ joint formed at $900{ }^{\circ} \mathrm{C}$ under $1 \mathrm{MPa}$ for $30 \mathrm{~min}$, (c) Inconel 600/Ag/SiC joint formed at $930{ }^{\circ} \mathrm{C}$ under $10 \mathrm{MPa}$ for $180 \mathrm{~min}$, and (d) Inconel $600 / 75 \% \mathrm{Ag}-25 \% \mathrm{Pd} / \mathrm{SiC}$ joint formed at $930{ }^{\circ} \mathrm{C}$ under $10 \mathrm{MPa}$ for $180 \mathrm{~min}$. The most relevant observation was made when comparing image (a) to (c) since the resultant microstructures observed are very different but the formation process used was the same and the only different was the presence of Pd concentration within image (c) interlayer.

Figure 41

Tensile test results for joints fabricated with both $\mathrm{Ag}$ as the interlayer (blue) and Ag-Pd 25\% as the interlayer (orange).

Figure 42 X-ray diffraction data of fracture surfaces of a joint formed at $900{ }^{\circ} \mathrm{C}$ under $1 \mathrm{MPa}$ for 30 minutes with the $\mathrm{Ag}$ interlayer. The top pattern is from the $\mathrm{SiC}$ fracture surface side and the bottom pattern is from the $\mathrm{Ag}$ Inconel 600 side.

Figure 43 X-ray diffraction data of the fracture surface of the joint formed at $930{ }^{\circ} \mathrm{C}$ under $10 \mathrm{MPa}$ for 180 minutes with the $\mathrm{Ag}$ interlayer. The top pattern is from the $\mathrm{SiC}$ fracture surface side and the bottom pattern is from the Inconel 600 fracture surface side. Many phases are present within both patterns, but most prominent are $\mathrm{NiSi}, \mathrm{Ni}_{5} \mathrm{Si}_{2}$ and $\mathrm{Ni}_{3} \mathrm{Si}_{2} \ldots \ldots \ldots \ldots \ldots \ldots . . . . . . . .72$

Figure 44 The tensile fracture surfaces of the joint fabricated at $930{ }^{\circ} \mathrm{C}, 10 \mathrm{MPa}$, and 30 min with Ag interlayer. ......................................................... 73

Figure 45 Microhardness data comparison for joints with Ag interlayers. The SEM image shown is from a joint fabricated at $930{ }^{\circ} \mathrm{C}, 10 \mathrm{MPa}$ and $30 \mathrm{~min}$, represented by the blue line.

Figure 46

Microhardness data comparison for all joints with $75 \%$ Ag-25 \% Pd interlayers on the cross-section of the joint. The SEM image shown is for a joint fabricated at $900{ }^{\circ} \mathrm{C}, 1 \mathrm{MPa}$, and $30 \mathrm{~min}$, represented by the black line.

Figure 47 The SEM image of the joint fabricated in this work, showing the methodology used to measure the diffusion thickness using image software such as Fiji or ImageJ. The thickness measurements are from the Inconel 600 to $\mathrm{SiC}$ interface to where the diffusion layer c' or c end...... 77

Figure 48 The plot of the diffusion layer thickness vs time ${ }^{1 / 2}$ for the SiC and Inconel 600 diffusion bonded transitions. Joints were formed at $930{ }^{\circ} \mathrm{C}$ under 10 MPa for times of 30, 60, 120, and 180 minutes. 
Figure 49 Crystal structure of possible interfacial silicide compounds from the diffusion bonding of SiC to Inconel 600. Structures modeled with CrystalMaker.

Figure 50 Phase diagrams of diffusion couples considered when indexing phases based on concentration ratios from the EDS point scans [60].

Figure 51

The formation sequence of reaction layers at the interface of joints fabricated at different combinations of process parameters. Each reaction layer is color-coded to correspond to a composition from the EDS point scans. The composition of the phases is determined by the concentration ratio of each element present in the regions and the expectations from a phase diagram plot.

Figure 52 The formation sequence of reaction layers at the interface of joints fabricated at $900{ }^{\circ} \mathrm{C}$ under $1 \mathrm{MPa}$ for $30 \mathrm{~min}$ and at $930{ }^{\circ} \mathrm{C}$ under $10 \mathrm{MPa}$ for $180 \mathrm{~min}$. Each reaction region is color-coded and relates to a specific concentration from the EDS point scans taken. The ratio extrapolated from these concentrations suggested the identification of specific phases........ 98

Figure 53 Plot of thickness vs square root of time of process performed at different temperatures to evaluate their correlation and extrapolate the rate constant that describes the progress of the process and understand how it depends on temperature. The rate constant is the slope of the fitted lines squared.

Figure 54 Plot of natural log of $\mathrm{k}$ vs $1 / \mathrm{T}$ to evaluate the correlation and extrapolate the activation energy that the process required. The activation energy is the slope of the fitted lines squared.

Figure 55 An Arrhenius plot of the $\mathrm{Al}_{4} \mathrm{Cu}_{9}, \mathrm{AlCu}, \mathrm{Al}_{2} \mathrm{Cu}$, and total intermetallic layer growth from [77]. 



\section{CHAPTER ONE: INTRODUCTION}

\section{1: Motivation for Research}

Advanced ceramic/metal structures created by diffusion bonding can be used in applications where high strength joints, corrosion resistance, hermetic seals and optimum properties at high temperature are required [1-5]. Ceramic/metal structures allow for increased operating temperatures, which results in greater thermodynamic efficiency in energy conversion systems or other systems such as (steam generators, nuclear plants, heat exchangers, etc.). The greater efficiency decreases the need for cooling while reducing the weight penalty $[2,6]$.

The lack of adequate techniques for joining ceramic to metal has limited the use of these structures. Diffusion bonding is a joining technique that can result in strong bonds through solid-state diffusion without the need of a liquid phase to bond the base materials [7]. Diffusion bonding allows the joining of dissimilar materials either directly or using interlayers. The types of joints that can be fabricated by diffusion bonding are: i) identical materials, ii) identical materials with an interlayer, iii) dissimilar materials, and iii) dissimilar materials with an interlayer.

This study investigates the diffusion bonding of SiC to Inconel 600 using two different interlayers: silver (Ag) and silver-palladium alloy (75\% Ag $25 \% \mathrm{Pd}$ ). Silicon carbide (SiC) bonded to Inconel 600 is a promising structure for extreme environment applications (high temperature and pressure). $\mathrm{SiC}$ has excellent oxidation properties and Inconel 600 maintains its mechanical properties at high temperature, resulting in robust/versatile assemblies. The joining of these two components to each other takes advantage of the positive characteristics of each material and has been a topic of interest 
in many recent research studies [8-15]. This study introduces an interlayer material into the ceramic/metal structure to reduce residual stresses and decrease the chances of cracking in the ceramic component. Ag and $75 \% \mathrm{Ag} 25 \% \mathrm{Pd}$ were the materials chosen as the interlayer components for this study since both interlayers are expected to improve the mechanical properties and optimize the reliability of the resultant joints. The ductility of $\mathrm{Ag}$ can accommodate the stresses that occur in the joining process due to the difference in coefficient of thermal expansion (CTE) of SiC and Inconel. Also, Ag is not expected to react with the $\mathrm{SiC}[8]$. The $75 \% \mathrm{Ag}-25 \% \mathrm{Pd}$ interlayer allowed for an increase in operational temperature of the joint since its melting point is greater than the melting point of silver. In addition, the concentration of Pd decreases the ductility of the interlayer, increasing its stiffness and resistance to deformation. To our knowledge, research on these two possible material system combinations has not been published.

\section{2: Research Objectives}

Despite numerous experimental studies, bond formation at the interfaces of different ceramic/metal material systems are not well understood [16]. The usefulness of a given specific ceramic/metal combination in structural applications is determined by the physical and mechanical properties of the ceramic/metal joints. These properties can only be optimized if the interfacial solid-state reactions that form between the ceramic and metal are understood. The interfacial microstructure is determined by the starting materials and the processing parameters. Therefore, a clear understanding of the relationship between processing, microstructure and properties is essential in order to utilize a ceramic/metal joint in a practical application. 
The overall purpose of this study is the fabrication, characterization, and understanding of diffusion-bonded $\mathrm{SiC} / \mathrm{Inconel} 600$ joints with $\mathrm{Ag}$ or $\mathrm{Ag}_{75} \mathrm{Pd}_{25}$ as interlayers. The specific goals are:

1) Evaluate the effect of process parameters on $\mathrm{SiC} / \mathrm{Inconel} 600$ interfacial microstructure.

2) Study the effect of interlayer material on the SiC/Inconel 600 interfacial solidstate reactions.

3) Evaluate the effect of interfacial solid-state reactions on the mechanical integrity of the joints in diffusion bonded SiC/Inconel 600 .

\section{3: Materials and Bonding Type}

Materials affect every aspect of our lives and are greatly referenced in all cultures [17]. The impact is consolidated on "transportation, housing, clothing, communication, recreation, and food production" [18]. Ceramics and metals are two of the most common classes of materials that shape every technology, from ancient times to the present. Metals have shaped the world's history and provided society with new tools, structures and technological devices. Across history, materials have driven technological improvements: from bronze, the first metal that humans used for tools and weapons such as axes, to the iron and steel revolution, and to more recently, the discovery of aluminum, titanium, and semiconductor materials as well as to the discovery of plastics and their wide range of usage.

Atomic arrangements and interactions between atoms and molecules define some of the important properties of materials. Simultaneously, these interatomic bonds are 
influenced by the outermost valence electrons [19]. Three different types of primary or chemical bond exist: ionic bonding, covalent bonding and metallic bonding. Atoms combine to form stable molecules and condensed phases by lowering the Gibbs free energy compared to the set of isolated atoms. When atoms interact with each other, forces of attraction and repulsion occur because of the interactions between the positively charged atomic nucleus and the negatively charge valence electrons [18]. All the types of bonds that form between atoms involve the valence electrons.

The interatomic interactions between atoms are influenced by the atomic distances between atoms. At large distances the interactions are neglected because the mutual influences between atoms cannot happen. Stable combinations occur at small atomic distances when the net attractive force $\left(\mathrm{F}_{\mathrm{A}}\right)$ of the electrons over the nucleus and vice versa is larger than the repulsive forces $\left(F_{R}\right)$. The bond strength is defined by the following equation 1.1 :

$$
\mathrm{F}_{\mathrm{N}}=\mathrm{F}_{\mathrm{A}}+\mathrm{F}_{\mathrm{R}}
$$

Metals and alloys are typically composed of one or more metallic elements and often, nonmetallic elements in relatively small amounts. Metallic bonding is found in metals and their alloys. In metallic bonds, all the atoms share their valence electrons. The nucleus forms a positively charge array bathed in a "sea" of delocalized electrons. Figure 1 shows a relatively simple representation of metallic bonds "sea of electrons". 


\section{METALLIC BONDING}

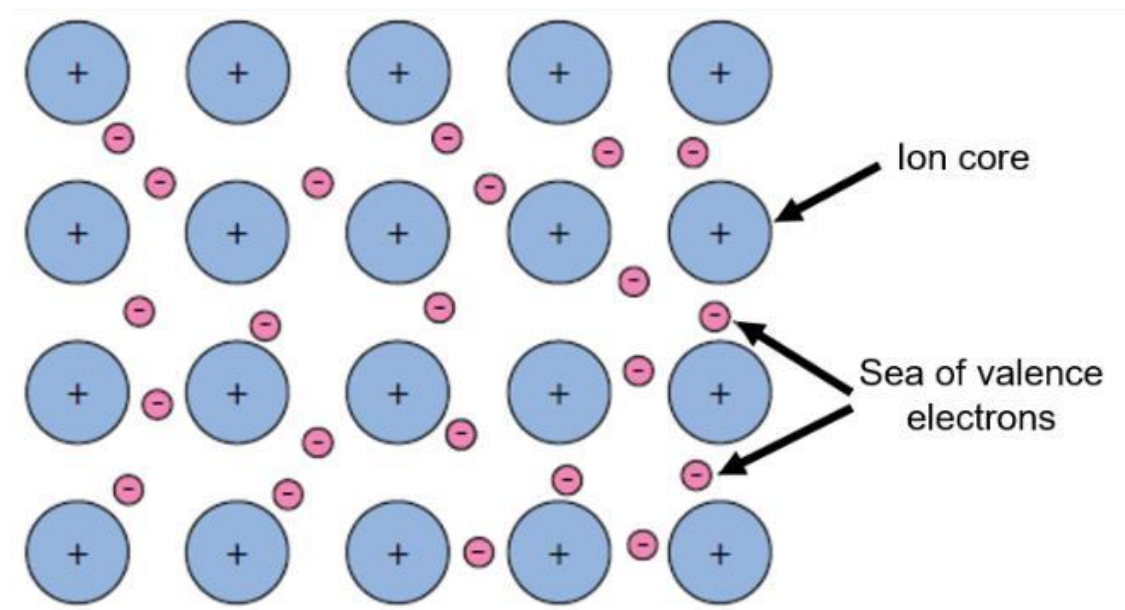

Figure 1 Schematic illustration of metallic bonding showing the "sea of electrons" also known as the "electron cloud". Image modified from [18].

Metals and alloys are very important for engineering applications because of the diverse properties that they possess, including good thermal and electrical conductivity, ductility and excellent mechanical strength [17]. Several other favorable characteristics of metallic materials are high sheen, hardness, resistance to corrosion, malleability, and magnetism. These properties are due to the way in which these atoms are arranged in the lattice and the characteristics of the metallic bond. By controlling the composition and the microstructure, the mechanical properties can be engineered specifically to fulfill the requirements related to the applications of interest.

While metals have been, and continue to be, widely used in engineering applications, ceramics are used extensively to provide thermal and electrical insulation and are also important structural materials. Ceramics are compounds between metallic and nonmetallic elements [18]. Ceramics display both ionic and covalent bonding. The relative degree of the ionic and covalent character of the bond in ceramic materials can be related to the electronegativity of its components. Electronegativity is defined as the 
tendency of an atom to attract electrons toward itself [19]. The percentage of ionic character is given by equation 1.2 :

$$
\text { \% Ionic Character }=\left(1-\exp \left[-0.25\left(X_{A}-X_{B}\right)^{2}\right]\right)
$$

When two bonding atoms have the same electronegativity, the valence electrons are shared equally, and the bond is purely covalent. However, when two atoms have very different electronegativity values, then the more electronegative atom draws nearly all of the valence electron density and the bond is purely ionic. Ceramics are neither purely ionic nor purely covalent, and can be classified by the degree of ionicity or covalency influenced by the electronegativity of the components $[3,18]$. In general, the ionic character of the bonds in ceramic materials is given by:

$$
\text { Oxides } \rightarrow \text { Nitrides } \rightarrow \text { Carbides (e.g. SiC) } \rightarrow \text { Borides }
$$

Ionic bonding is always found in compounds composed of both metallic and nonmetallic elements. Atoms of a metallic element tend to give up their valence electrons to the nonmetallic atoms, until all of the atoms have a stable electronic configuration. Figure 2 represents the ionic bonding of a sodium chloride molecule. 


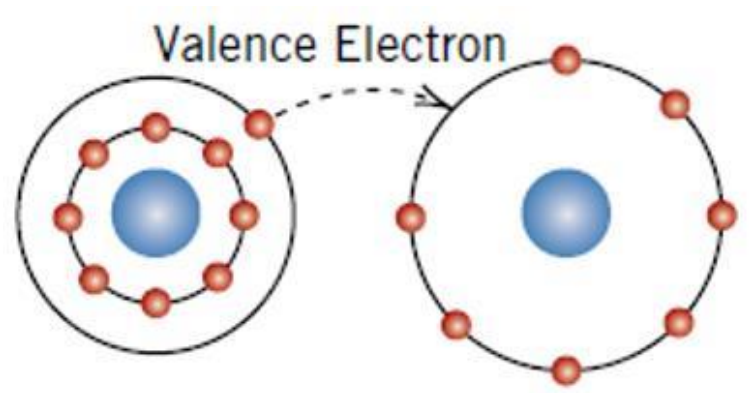

Na Atom

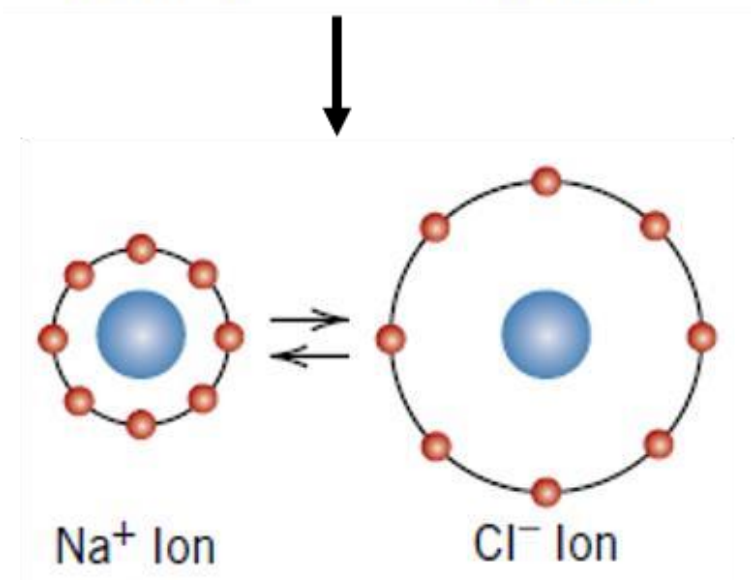

Figure 2 Schematic representation of (a) the combination of $\mathrm{Na}^{+}$and $\mathrm{Cl}^{-}$ions forming $\mathrm{NaCl}$, which is a classic ionic bonding case [18].

In covalent bonding, a stable electron configuration is acquired by the sharing of electrons between adjacent atoms [18]. Two atoms that are covalently bonded will each contribute at least one electron to the bond, and the shared electrons may be considered to belong to both atoms. Figure 3 demonstrates the illustration of a covalently-bonded $\mathrm{CH}_{4}$ molecule. The carbon atom and the hydrogen atoms that constitute the molecule shared their valence.

The ionic and covalent bonding in ceramics contributes to the typical mechanical properties of low ductility, and highly susceptibility to brittle fracture [18]. These properties limit the use of ceramics in engineering applications. Newer ceramics are being engineered to have improved resistance to fracture, yet still are limited in their use by the lack of ability to form complex shapes. One solution to the limitations of both 
ceramics and metals is to combine the two components to take advantage of the best properties of each.

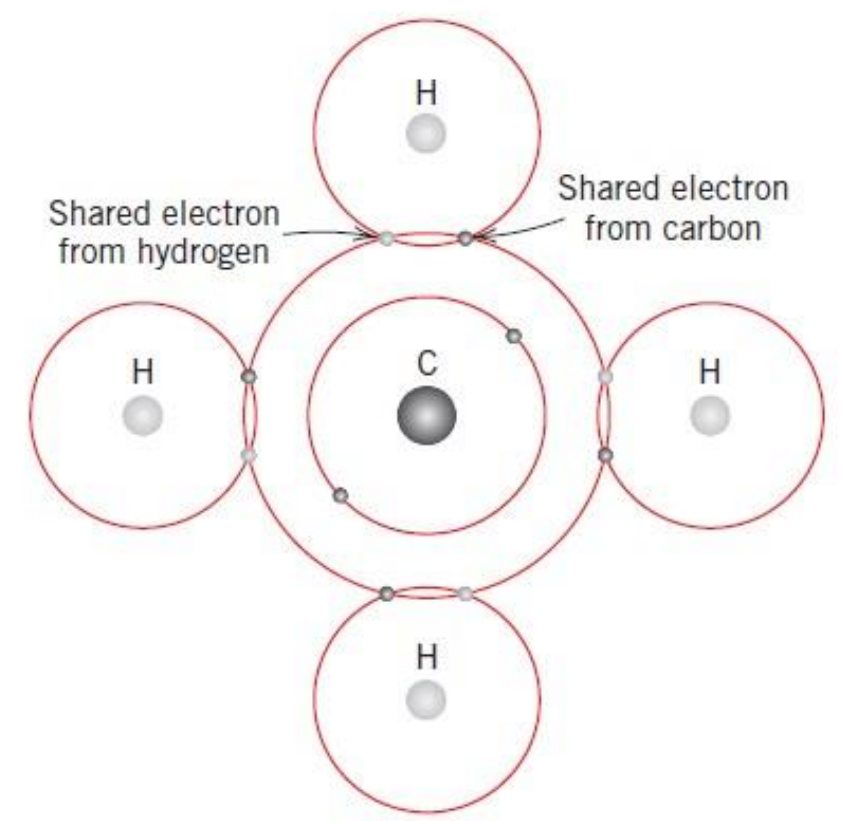

Figure 3 Schematic representation of covalent bonding in a molecule of methane $\left(\mathrm{CH}_{4}\right)$ illustrating the sharing of valance electrons between atoms [18].

\section{4: Joining Processes}

In general, ceramic/metal joints are created through three types of processes. The first type is known as mechanical joining and is achieved through the use of the mechanical interlocking of components. This category includes screwing, fitting, and clamping. The second type is known as indirect joining; where joining is completed through an intermediate layer of material, such as an adhesive material, cement, or a braze. The third and last type of joining mechanism is direct joining. In direct joining, components are created by solid-state diffusion, laser bonding, or fusion bonding $[3,20]$. Figure 6 shows some of the methods and how joining procedures are classified based on the characteristics of the process. 


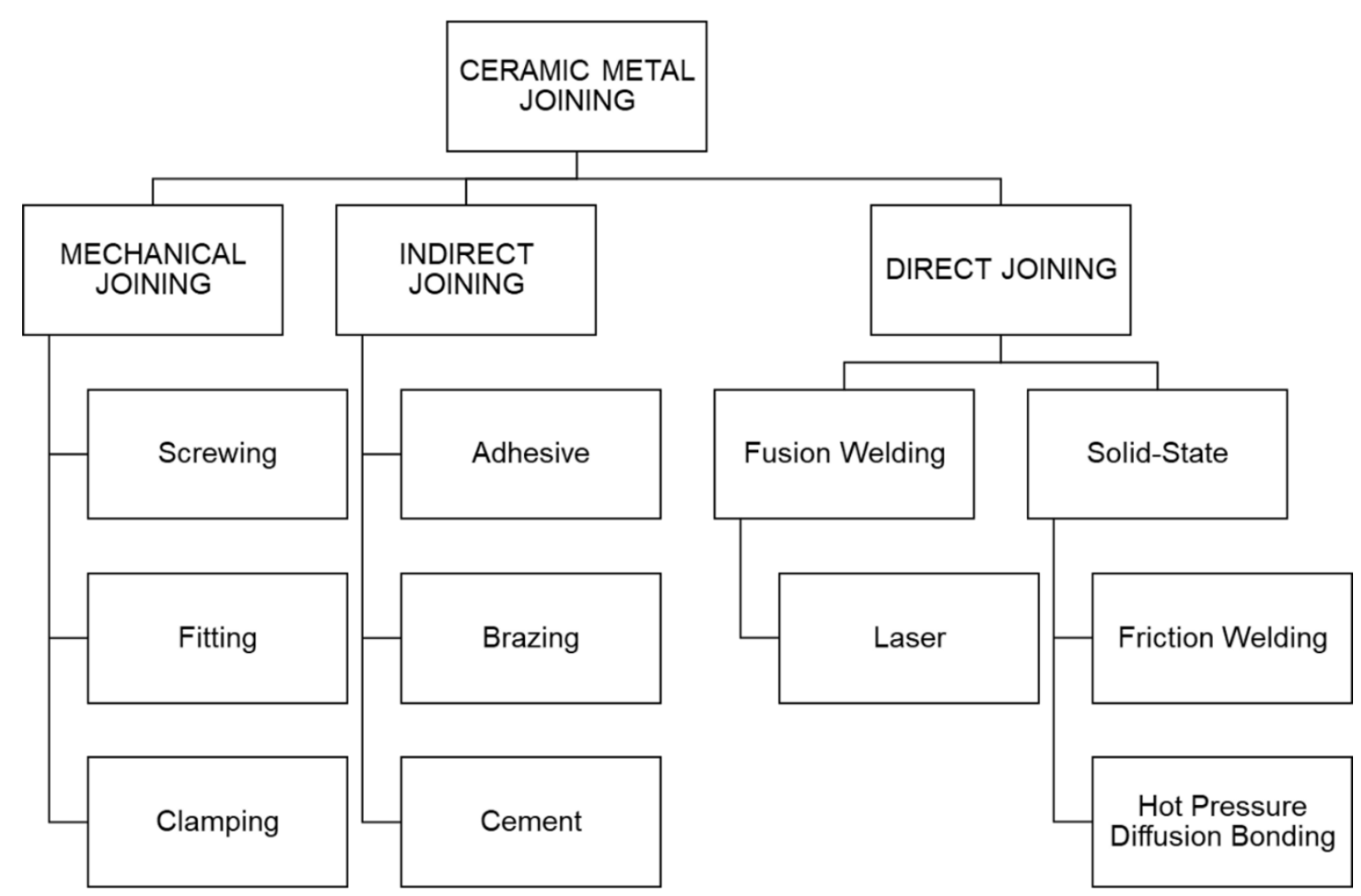

Figure 4 Schematic of the different possible joining techniques for ceramic/metal joints $[3,20]$.

In this study, a form of direct bonding, solid-state diffusion bonding was used.

Solid-state diffusion bonding is a solid-state process for the fabrication of metal-metal, ceramic-ceramic, and ceramic-metal joints. Diffusion bonding does not require melting of any of the components and in general, occurs at higher temperatures than other types of joining. Joints are formed at high temperatures (50-95\% of the melting temperature of the lowest melting point material within the system) while applying a constant pressure for a specified time $[3,18,21]$.

Diffusion bonding includes processes where the ceramic is joined to the metal with or without an interlayer component. Successful joining relies upon the achievement of adequate interfacial contact to eliminate interfacial porosity and surface contact voids and to allow formation of an interfacial layer. When ceramic-metal joints are being formed, plastic deformation generally is expected to happen within the metal. The 
advantages of direct pressure bonding are the simplicity, as it is generally a single-step process, the potential to achieve very high joint strength, and the limited amount of deformation that occurs. Because it is a low deformation process, complex or sensitive parts can be joined without much surface distortion. However, the limitations and disadvantages must also be considered such as high applied pressure, and the need for a controlled environment, typically an inert atmosphere or under vacuum, adding complexity and cost to the equipment required for bonding.

The properties of the interfacial layer formed in ceramic/metal joints have a significant effect on the integrity and mechanical properties. The bonding mechanism between components can be defined as a function of mass transfer or charge transfer across the interface [21]. Consequently, bonding between ceramic/metal components results from chemical/physical interaction or from a chemical reaction resulting in a new phase. In the initial stage of the bonding process, a physical bond is created by the charge transfer across the interface, resulting in van der Waals forces between the materials [21, 22]. The driving force for formation of ceramic/metal interfaces is the decrease in free energy $(\Delta G)$ when intimate contact is established between surfaces. The $\Delta \mathrm{G}$ is given by the Dupré equation 1.3: $\Delta G=\gamma_{M}+\gamma_{C}+\gamma_{M C}$

Where $\gamma_{M}, \gamma_{C}$ are the surface energies of the metal and ceramic, and $\gamma_{M C}$ is the metal/ceramic interfacial energy.

Figure 5 shows (A) charge transfer between ceramic/interlayer/metal components of the system resulting in a physical bond and (B) the schematic representation of 
metal/ceramic contact (i) the initial contact or rigid solid surface and (ii) the final equilibrium configuration on the deformable solid [21].

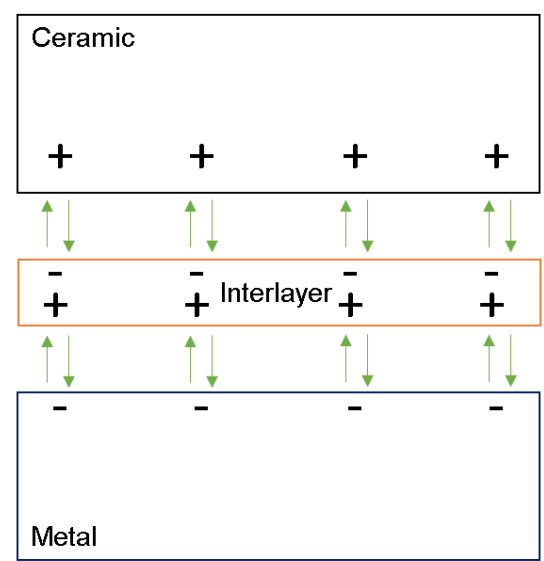

(A)

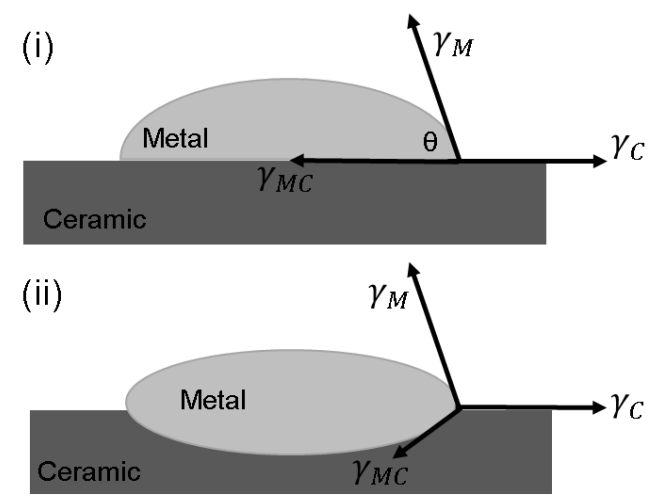

(B)

Figure 5 (A) Illustration of chemical/physical bond by charge transfer across each component resulting in van der Waals bonding. (B) Liquid metal drop shape depending on contact time: (i) initial contact or solid surface contact and (ii) equilibrium arrangement on deformable solid surface. Image modified from [21, 22].

Diffusion bonding occurs when mass transfer across the interface is present. The bonding can be a result of just diffusion or of diffusion with a chemical reaction. A chemical reaction will result in new phases formed in the interfacial region. These phases can have properties that differ from both the ceramic and the metal and may improve or degrade the strength and integrity of the joint. The driving force for diffusion is the composition gradient across the atomic species involved. A chemical reaction is thermodynamically favorable when the Gibbs free energy is reduced $(\Delta \mathrm{G})$ relative to the free energy of the individual components of the base materials. Equilibrium thermodynamics can be used to predict possible reactions at the interface. However, when there are more than three elements in a ceramic-metal system, the prediction of all the possible reactions based on the phase diagrams is difficult. 
Phase formations and the thermodynamics of the system are further complicated by the use of an interlayer in the bonding process. Often a ceramic to metal joint uses a ductile intermetallic-interlayer that provides a stress gradient, or buffer, between the typical low CTE ceramic and higher CTE metal. Additional phases are often observed when using this technique between the base ceramic, interlayer, and the metal components. Often these phases are brittle intermetallic compounds (e.g. nickel silicide) that weaken the joint. It may be possible to limit formation of deleterious new phases through appropriate selection of interlayer materials and thicknesses as well as a specific combination of processing conditions. Materials selection for the different components to be bonded needs to address the reactivity between materials, as well as the CTE mismatch.

These interlayers may also assist in achieving bonding at reduced temperatures and pressure. Soft interlayers with good yield characteristics enhance contact by closing the voids present at the interface, while accommodating the residual stresses developed at the interface. Figure 6 shows a schematic representation of the diffusion bonding process between two dissimilar materials.

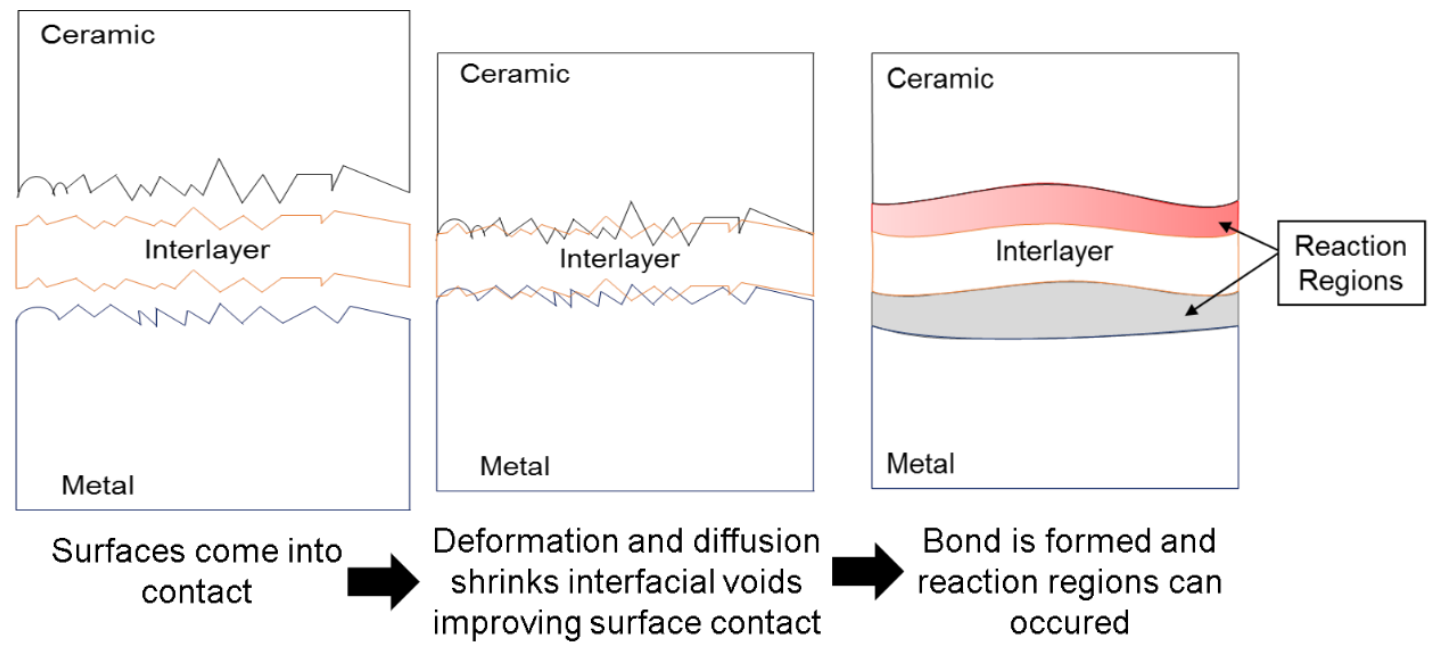




\section{Figure 6 Diffusion bonding sequence illustration of ceramic/metal diffusion bonded joints with metallic interlayer. Image modified from [21].}

While thermodynamics helps to predict the likeliness of formation of the expected solid-state reaction phases at the interface, kinetics helps to understand how fast the process going from initial stage to final stage occurs. Diffusion is a migration process where mass-transfer occurs across an interface due to a concentration gradient. To know the diffusion rate, the diffusion flux is considered following Fick's first law [18]. In mathematical form, this may be represented as:

$$
J=\frac{M}{A t}
$$

Where $\mathrm{J}$ is the diffusion flux, $\mathrm{M}$ is the mass that transferred, $\mathrm{A}$ is the area, and $\mathrm{t}$ is time.

The simplest approach to demonstrate the phenomenon of diffusion is via diffusion couples. Interdiffusion occurs when atoms of one metal transfer into the second metal in contact and vice versa This mechanism is also called impurity diffusion [18]. From an atomic perspective, there are two possible diffusion mechanisms: (1) vacancy diffusion, which is described as the interchange of an atom from a normal lattice site to an adjacent empty lattice site, also known as vacancy, and (2) interstitial diffusion, which is described as the migration of atoms from an interstitial site to a neighboring interstitial site. Figure 7 shows the different diffusion couples, which were considered to understand the kinetics processes resulting in diffusion bonding in the Inconel 600-Ag-SiSiC system. 


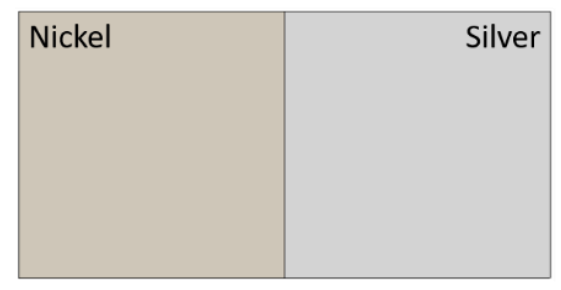

(a)

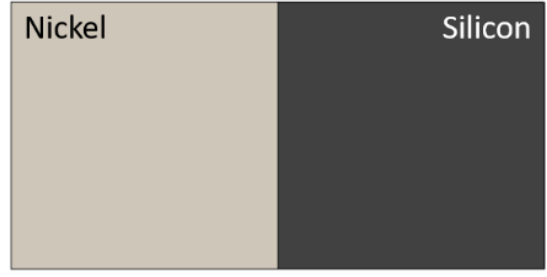

(b)

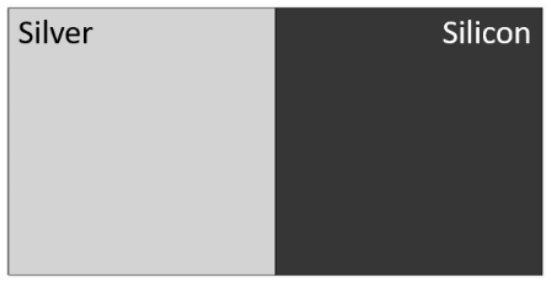

(c)

\section{Figure 7 Diffusion couples used to understand the fundamental diffusion processes that can occur due to the materials present within the Inconel 600- AgSiSiC system.}

Most practical diffusion situations are nonsteady-state [18]. This means the diffusion flux and the concentration gradient in a solid vary with time, resulting in a net accumulation or depletion of the diffusing species. The interfacial solid-state reaction region in this work grows parabolically with time, following Fick's second law for a nonsteady state diffusion process.

$$
\frac{d C}{d t}=\frac{d}{x}\left(D \frac{d C}{d x}\right)
$$

Where $\mathrm{C}$ is the concentration, $\mathrm{t}$ is time, $\frac{d C}{d x}$ is the concentration gradient, and $\mathrm{D}$ is the diffusion coefficient.

The most accepted expression of nickel (Ni) diffusivity in silicon ( $\mathrm{Si}$ ) has been $D_{N i}(T)=2.3 \times 10^{-3} \exp \left(-\frac{0.47}{k_{B} T}\right) \mathrm{cm}^{2} /$ s however base on a study done by J. Lindroos, et al. [23] where modern experimental methods a faster expression of Ni diffusivity in Si was found to be $D_{N i}(T)=(1.69 \pm 0.74) \times 10^{-4} \exp \left(-\frac{0.15 \pm 0.004}{k_{B} T}\right) \mathrm{cm}^{2} / \mathrm{s}$. Following the same idea, the diffusion of iron $(\mathrm{Fe})$ in $\mathrm{Si}$ is given the expression $D_{\mathrm{Fe}}(T)=9.5 \times$ 
$10^{-4} \exp \left(-\frac{0.65}{k T}\right) \mathrm{cm}^{2} / \mathrm{s}[24]$. Lastly, the diffusivity of silver $(\mathrm{Ag})$ in Si was extrapolated from Figure 8 [18].

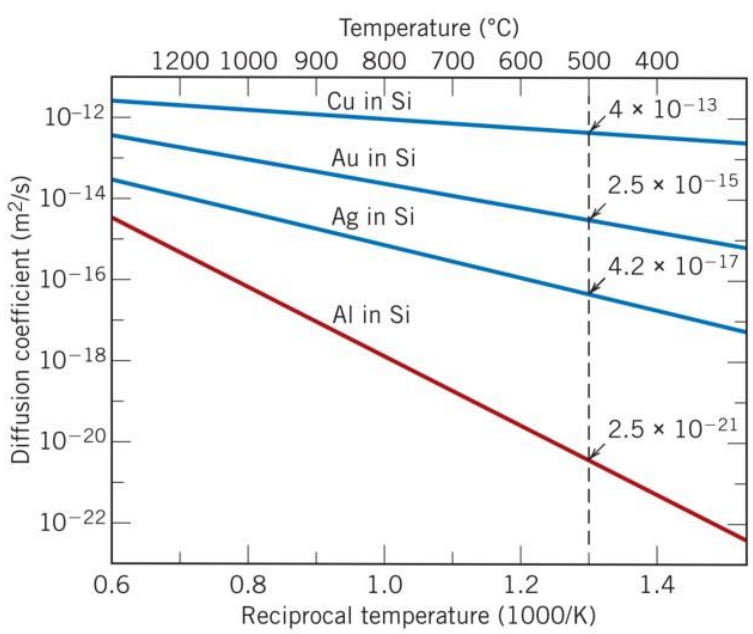

Figure 8 Logarithm of D-vs-1000/T lines for the diffusion of $\mathrm{Cu}, \mathrm{Au}, \mathrm{Ag}$, and $\mathrm{Al}$ in $\mathrm{Si}[18]$.

Although the materials system in this project is more complex than a simple diffusion couple, by modeling the system as a system in this way, a better understanding of what the concentration of each species could be diffusion from each component and forming an interface region. This concentration prediction can then help in determining the possible phases that may form in that region. For example, for a specific Ni silicide to form such as $\mathrm{Ni}_{3} \mathrm{Si}$, there must be a ratio of $1 \mathrm{Si}$ atom for every $3 \mathrm{Ni}$ atoms.

Numerous obstacles exist for a successful ceramic/metal joining. One of the main challenges in these engineered structures is the high residual stresses induced by the mismatch of the coefficient of thermal expansion (CTE) between metals and ceramics [25]. The CTE, as a function of temperature, for the materials considered in this project is shown in Figure 9. As seen in Figure 9, Inconel 600 has CTE values more than five times larger than $\mathrm{SiC}$. The mismatch of CTE causes significant strain in the joint as it is cooled 
from the forming temperature to room temperature. Significant strain also occurs due to fluctuation of temperature in use. One way to compensate is to use a much more ductile material for an interlayer, such as $\mathrm{Ag}$, to compensate for the strain.

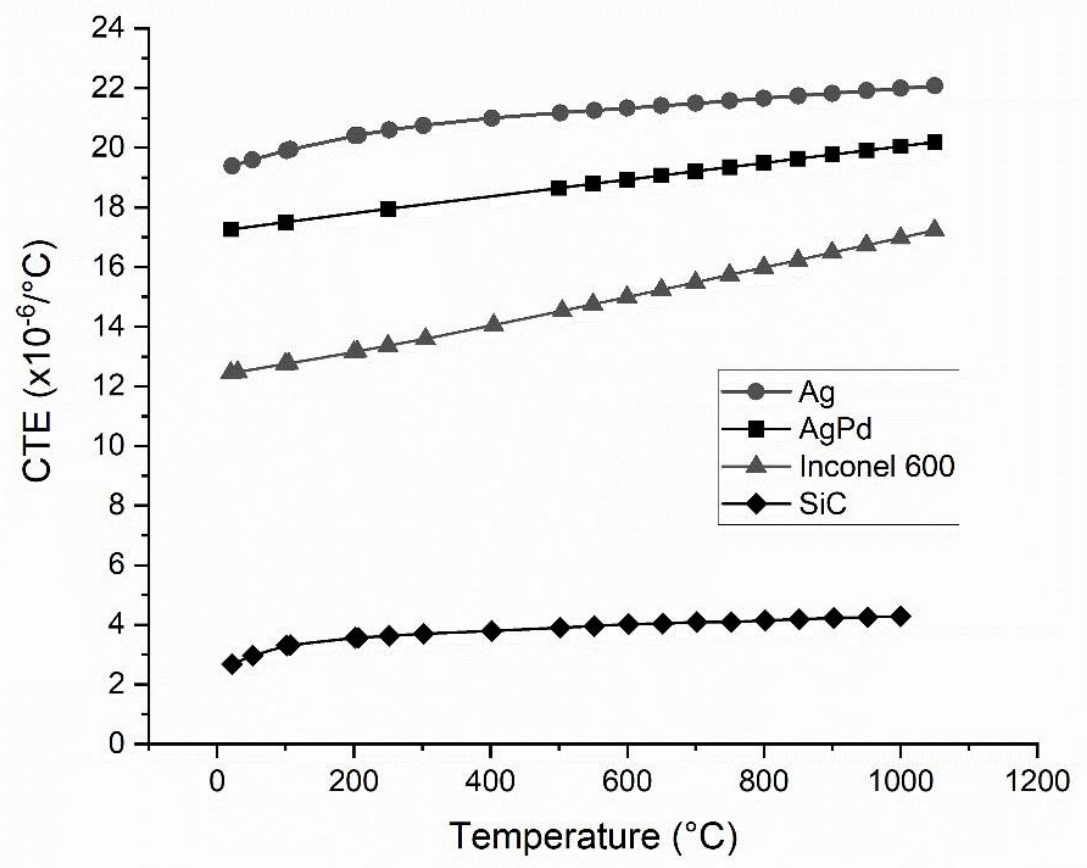

Figure 9 CTE of the different materials used within this study as a function of Temperature [26, 27].

Figure 10 shows an illustration of stresses at a joint interface and the cracking that can occur due to a CTE mismatch upon cooling. When the CTE of the ceramic is smaller than that of the metal, the ceramic is subjected to tensile stresses and cracks at the edges, as shown in Figure 10 (a). On the other hand, when the CTE of the metal is smaller than that of the ceramic, tensile stress acts on the core of the ceramic and cracks the ceramic, not at the edges but perpendicular to the metal surface, as shown in Figure 10 (b). 


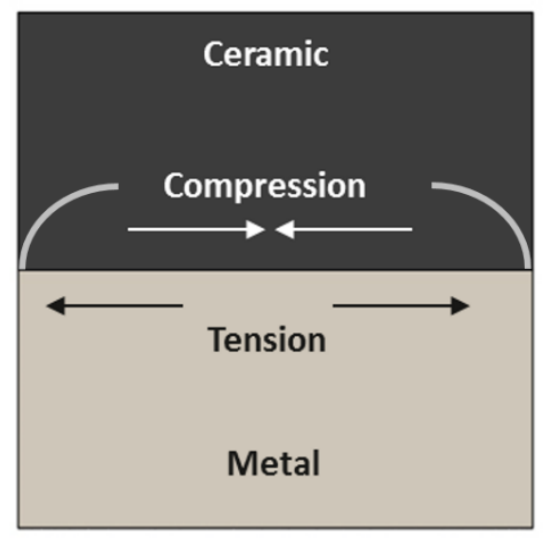

(a)

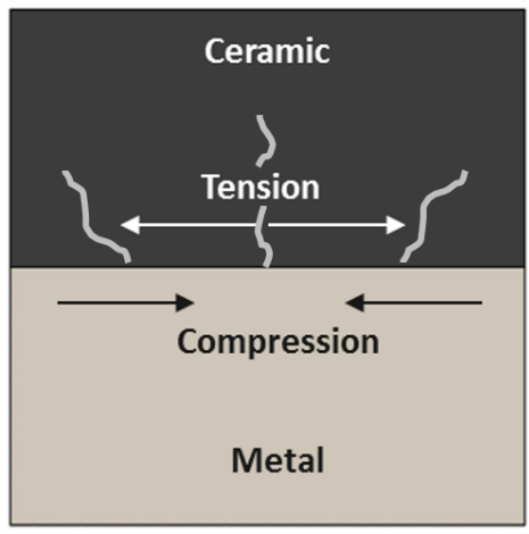

(b)

Figure 10 Schematic illustration of thermal stress in joint interface and mode of cracking due to the difference of the thermal expansion coefficient upon cooling; (a) Edge cracks in ceramic and (b) Core cracks in ceramic. If (a) mode happens the CTE of the ceramic < the CTE of the metal and if (b) happens the CTE of the ceramic > the CTE of the metal. Figure adapted from [21].

Most joining processes between metals and ceramics occur at relatively high temperatures. These temperatures affect the amount of expansion that the materials experienced during heating, dwelling, and cooling. In addition, the microstructure at the interface changes and new phases are formed. These new phases also expand and contract different amounts with temperature. As shown in Figure 10, (a) and (b) a localized region of high stress develops at the interface after joining and cooling, because of the CTE mismatch. This stress can lead to poor joint strength $[3,25]$. Structural cracking can occur, which decreases the performance and reliability of the overall structure and may result in hazardous conditions, loss of efficiency, or catastrophic events. These stresses can be relieved by two commonly applied methods $[3,21]$ :

1. Using a metal with a similar CTE as that of the ceramic to decrease the magnitude of stress generated at the interface. 
2. Introducing a ductile interlayer between the metal and the ceramic. The interlayer deforms due to its ductility, addressing the CTE mismatch.

Other methods to overcome the residual stress at the interface due to the CTE mismatch between the ceramic and metal components are [21]:

1. Using a composite interlayer where the composite interlayers are composed of hard, not commonly malleable metals and soft, more ductile metals to engineer the coefficient of thermal expansion and mismatch at the interface.

2. Joining using low temperatures to reduce the joint deformation and effectively decreasing the residual stresses.

3. Heat treatment after high temperature joining to release the stresses caused while cooling.

All of these methods can also be used in combination.

\section{5: Literature Review}

Diffusion bonding of ceramic to metals is not a novel technique. However, the complexity of the systems leaves many gaps in the understanding of the materials science of the joints and the optimal materials selection and processes. Table 1 summarizes published reports that have investigated solid-state diffusion to form ceramic/metal joints for high temperature applications. These results were also considered as a guide to the development and improvement of this work.

\section{Table 1 Relevant literature on diffusion bonding dissimilar materials for complex applications.}




\begin{tabular}{|c|c|c|c|}
\hline Material System & $\begin{array}{l}\text { Joining } \\
\text { Technique }\end{array}$ & $\begin{array}{c}\text { Chemical } \\
\text { Characterization }\end{array}$ & $\begin{array}{c}\text { Mechanical } \\
\text { Characterization }\end{array}$ \\
\hline $\begin{array}{c}\mathrm{SiC} / \mathrm{SiC} \text { using } \\
\mathrm{Cr}(\text { coat) \& Ni foil } \\
{[10]}\end{array}$ & SSD & $\begin{array}{l}\text { SEM/EDS \& } \\
\text { metallographic } \\
\text { analysis }\end{array}$ & None \\
\hline $\begin{array}{c}\text { SiC/Haynes } 214 \\
\text { using Ni \& Ag as } \\
\text { interlayer [8] }\end{array}$ & SSD & SEM/EDS and XRD & Shear Testing \\
\hline $\begin{array}{c}\text { RB-SiC/Inconel } 600 \\
\text { [9] }\end{array}$ & SSD & $\begin{array}{c}\text { SEM/EDS, XRD \& } \\
\text { WDS }\end{array}$ & Shear Testing \\
\hline $\mathrm{SiC} / \mathrm{Fe}[13]$ & SSD & $\begin{array}{c}\text { SEM/EDS, XRD \& } \\
\text { EPMA }\end{array}$ & $\begin{array}{c}\text { Microhardness Vickers } \\
\text { Testing }\end{array}$ \\
\hline $\begin{array}{l}\text { SiC/Steel using W- } \\
\text { Pd-Ni interlayer [15] }\end{array}$ & SSD & $\begin{array}{l}\text { SEM/EDS \& } \\
\text { EPMA }\end{array}$ & $\begin{array}{c}\text { Nanoindentation } \\
\text { Hardness, Shear Testing, } \\
\text { and 4-point Flexure } \\
\text { Testing }\end{array}$ \\
\hline $\begin{array}{c}\text { SiC/Steel using } \\
\text { W/Ni as interlayer } \\
{[14]}\end{array}$ & 2-SSD & SEM/EDS \& XRD & $\begin{array}{l}\text { Nanoindentation Hardness } \\
\text { and Tensile Testing }\end{array}$ \\
\hline $\begin{array}{c}\text { RB-SiC/Inconel } 600 \\
\text { with Ni-based } \\
\text { brazing alloy }\left(\mathrm{BNi}_{5}\right) \\
{[12]}\end{array}$ & HTB & $\begin{array}{l}\text { Optical micrograph } \\
\text { \& SEM/EDS }\end{array}$ & None \\
\hline
\end{tabular}

Where SSD stands for Solid-State Diffusion, 2-SSD stands for 2 Step Solid-State Diffusion, and HTB stands for High Temperature Brazing.

The literature presented in Table 1 covers a wide variety of material systems and hence served as a guide for this work, especially the characterization of interfaces, process parameters determination, and mechanical testing techniques. Below, each study is explained briefly.

K. Bhanumurthy and R. Schmid-Fetzer [10] demonstrated that joining hot isostatically pressed $\mathrm{SiC}$ to $\mathrm{SiC}$ was possible at relatively low temperatures. The bonding 
involved a pre-bonding step where the $\mathrm{SiC}$ components were covered with $\mathrm{Cr}$ powder and annealed at $1,000{ }^{\circ} \mathrm{C}$ for $1-600$ hours. The pre-bonding process produced the thermodynamically stable ternary phase $\mathrm{Cr}_{5} \mathrm{Si}_{3} \mathrm{C}$. The two components were then diffusion bonded using $\mathrm{Ni}$ foil as an intermediate layer, under a maximum pressure of 15 MPa. Multiple bonding trials were performed in the temperature range of $700-1000{ }^{\circ} \mathrm{C}$ for 2-6 hours. Extensive reactions resulting in new phases and the total consumption of the $\mathrm{Cr}$ coated layer was observed. The nickel reacted with the $\mathrm{SiC}$ forming Ni-silicides and elemental carbon and the interactions of $\mathrm{Ni}$ and $\mathrm{Ni}-\mathrm{Cr}$ alloys with $\mathrm{SiC}$ led to the formation of complex interlayers and new ternary compounds [10].

The work done by M.L. Hattali, et al. [8] investigated the formation of brittle silicide phases at the interface of $\mathrm{SiC} /$ metal bonds using a $\mathrm{Ni}_{0.93} \mathrm{~B}_{0.07}$ coating, an $\mathrm{Ag}$ coating, and a Ag foil. The authors demonstrated that the addition of boron does not inhibit the formation of brittle phases at the interface. Secondly, the authors demonstrated that the use of an Ag coating of $200 \mu \mathrm{m}$ thickness eliminates the reaction of $\mathrm{SiC}$ with $\mathrm{Ni}$. However, the heterogeneity of the coating after bonding and the diffusion of Ni through the coating led the authors to conclude that the thickness must be greater than $50 \mu \mathrm{m}$ to effectively prevent a Ni-SiC reaction at high temperatures. Finally, the authors demonstrated that the bonding reactions between $\mathrm{SiC}$ and $\mathrm{Ni}$ were eliminated using an $\mathrm{Ag}$ foil of thickness $200 \mu \mathrm{m}$.

Junqin Li and Ping Xiao [9] investigated the chemical reactions that occurred at the reaction bonded silicon carbide (RBSC)/Inconel 600 bonded interface between 900 ${ }^{\circ} \mathrm{C}$ and $1,080{ }^{\circ} \mathrm{C}$, and related the microstructure of the interfaces to the mechanical behavior of the joints. The authors determined that the formation of reaction layers at the 
interface were controlled by the joining temperature. In addition, the authors suggested that the distribution of silicide phases and the formation of large areas of pores in the interfacial region occurred because of the existence of a transient liquid phase during joining. The reaction between the liquid Ni-Si alloy and $\mathrm{SiC}$ depended on the Si content. The reaction products observed in this study were mainly Ni- or Cr-based silicide phases with dissolved Fe. Diffusion of Si occurred during the joining process and led to the formation of pores in the SiC component. The authors concluded that at higher temperatures $\left(1,080^{\circ} \mathrm{C}\right)$ the formation of liquid $\mathrm{Ni}$-Si alloy and the precipitation of solid phases eliminated the formation of pores in the TBSC, leading to the formation of strong joints.

The work done by W. M. Tang et al. [13] studied the solid state reactions between $\mathrm{SiC}$ and $\mathrm{Fe}$ annealed in $\mathrm{Ar}-20 \% \mathrm{H}_{2}$ atmosphere in the temperature range from $800-1,100$ ${ }^{\circ} \mathrm{C}$ for times from $0.5-40$ hours. Fe-silicide phases such as $\mathrm{Fe}_{3} \mathrm{Si}, \mathrm{Fe}(\mathrm{Si})$ and graphitic carbon precipitates were observed. The reaction $3 \mathrm{Fe}+\mathrm{SiC} \rightarrow \mathrm{Fe}_{3} \mathrm{Si}+\mathrm{C} \mathrm{Gr}$ described the

$\mathrm{SiC}-\mathrm{Fe}$ interaction and was consistent with thermodynamic expectations. The mechanical property of the individual layers in the reaction region formed at $1,100{ }^{\circ} \mathrm{C}$ for 3 hours was evaluated with Vickers hardness testing. The layer of pure Fe had the lowest hardness value as expected, and the SiC layer had the highest hardness, as expected. In the other reaction layers, the authors concluded that the value of microhardness decreased with the increasing carbon content and decreasing Si content. The reaction followed the parabolic growth law indicating the reaction was limited by diffusion. The reaction rate 
constant was reported as $4.9010^{-4} e^{(-(180) 103) / R T)} \mathrm{m}^{2} \mathrm{~s}^{-1}$ where $\mathrm{R}$ is the ideal gas constant and $\mathrm{T}$ is temperature. The activation energy of the diffusion of $\mathrm{Fe}$ in $\mathrm{Fe}_{3} \mathrm{Si}$ was found to be $180 \mathrm{~kJ} / \mathrm{mol}$ from the plot of the reaction rate constant versus the reaction temperature for the reaction between $\mathrm{SiC}$ and $\mathrm{Fe}$, indicating that $\mathrm{Fe}$ diffusion in the Fesilicide phase is the dominating diffusion species of the reaction.

Zhihong Zhong, et al. [15] studied the bonding between SiC and stainless steel (SS) with a W-Pd-Ni interfacial layer to promote and broaden the practical applications of SiC. The authors demonstrated successful joining between SiC and SS using a W-PdNi interlayer. The bonding conditions used were a temperature range of $1,250-1,350{ }^{\circ} \mathrm{C}$ for 30-120 min under flowing argon under $20 \mathrm{MPa}$. However, extensive interfacial reactions were observed. All the elements from the interlayer reacted with the $\mathrm{SiC}$, forming $\mathrm{Pd}, \mathrm{Ni}$, and $\mathrm{W}$-silicide phases. The authors explained the reason was the chemical affinity of Si for the metal elements. Two chemical reactions were presented:

$$
\begin{gathered}
\mathrm{SiC}+2 \mathrm{PdO} \rightarrow \mathrm{Pd} \mathrm{Si}_{2}+\mathrm{C} \\
\Delta G=-154.9 \mathrm{~kJ} / \mathrm{mol} \\
\mathrm{SiC}+2 \mathrm{Ni} \rightarrow \mathrm{Ni} \mathrm{Si}+\mathrm{C} \\
\Delta G=-69 \mathrm{~kJ} / \mathrm{mol}
\end{gathered}
$$

The Gibbs free energies of both reactions are negative, hence both reactions can occur spontaneously, as seen by the formation of reaction structure $\mathrm{SiC} / \mathrm{Pd}_{2} \mathrm{Si}+\mathrm{Ni}_{2} \mathrm{Si}+\mathrm{C}$ at the interface. Precipitation of $\mathrm{C}$ was predicted as well. The authors did not report any 
reactions at the $\mathrm{W}-\mathrm{Pd}-\mathrm{Ni} / \mathrm{SS}$ interface and reported good bonding at the metal-metal interface. The joining temperature and holding time did not have a noticeable effect on the interfacial microstructure. High hardness values in the reaction zone were attributed to the formation of silicide compounds. Shear testing indicated that the joints produced had a moderate strength and a potential usage at temperatures of around $600{ }^{\circ} \mathrm{C}$.

Another study done by Zhihong Zhong, et al. [14] described the microstructure and mechanical properties of diffusion bonded $\mathrm{SiC} /$ steel joint using a W/Ni interlayer. The interlayer was chosen to prevent the interfacial reactions that affect the strength of the joint and to reduce the residual stress in the joint. The diffusion bonding process was a two-step process. The first step consisted of the diffusion bonding of a SiC/W structure at $1,550{ }^{\circ} \mathrm{C}$ for 1 hour in argon under $20 \mathrm{MPa}$. The second step consisted of the diffusion bonding of the pre-bonded $\mathrm{W} / \mathrm{SiC} / \mathrm{W}$ structure to $\mathrm{SS}$ using a Ni interlayer to form the overall sequence of $\mathrm{SS} / \mathrm{Ni} / \mathrm{W} / \mathrm{SiC} / \mathrm{W} / \mathrm{Ni} / \mathrm{SS}$. The second step was carried out in the temperature range of $750-900{ }^{\circ} \mathrm{C}$ for 3 hours under a pressure of $3 \mathrm{MPa}$ and under vacuum $\left(10^{-3} \mathrm{~Pa}\right)$ conditions. Strong joints were successfully demonstrated by the twostep method. Interfacial solid-state reactions such as formation of a $\mathrm{W}$-silicide phase $\left(\mathrm{W}_{5} \mathrm{Si}_{3}\right)$ and $\mathrm{WC}$ were observed at the $\mathrm{SiC} / \mathrm{W}$ interface. $\mathrm{A} \mathrm{Ni}(\mathrm{W})$ solid solution was reported in the W/Ni interface for joints formed at temperatures below $850{ }^{\circ} \mathrm{C}$. At temperatures higher than $850{ }^{\circ} \mathrm{C}$, the intermetallic compound $\mathrm{Ni}_{4} \mathrm{~W}$ was observed. At the $\mathrm{Ni} / \mathrm{SS}$ interface, no change in elemental concentration was seen, suggesting that interfacial reactions were avoided. The hardness values were dependent on the reaction products and the strengthening effect of the formation of a solid solution. The authors reported the 
failure of joints consistently occurred at the SiC/W interface as result of the CTE mismatch between the $\mathrm{SiC}$ and the $\mathrm{W}$ metal.

The interaction of reaction-bonded $\mathrm{SiC}$ and Inconel 600 with a Ni-based brazing alloy was studied by J.T. McDermid, et al. [12]. The authors used direct brazing as the joining technique to form the ceramic/metal joint. Brazing requires the formation of a wetting liquid at the ceramic/metal interface. According to the authors, the brazing process should not significantly affect the properties of the base materials. Wetting of the ceramic by the liquid metal and the CTE mismatch are generally the most difficult problems to overcome. To overcome the effect of the CTE mismatch, a ductile metal that plastically deforms to account for the CTE mismatch-stresses is used. These ductile layers, known as brazes, tend to have low melting points and therefore they do not satisfy the projected operating temperatures of advanced heat engines. The authors considered $\mathrm{BNi}_{5}$ and $\mathrm{BNi}_{7}$ as the brazing alloys. The degradation of the starting materials was considered to be a result of $\mathrm{Ni}-\mathrm{SiC}$ reactions. Ni-silicide phases were observed, as well as the formation of $\mathrm{Cr}_{7} \mathrm{C}_{3}$ at the ceramic/metal interface. In addition, the authors observed cracking and failure at the ceramic-metal interface during cooling, due to the formation of the brittle Cr-carbide phase and the stresses induce by the CTE mismatch.

Although a variety of research studies have been completed, the complexity of the materials reactions has limited the fundamental understanding of the systems. Because of the wide variety of materials and applications, further studies are clearly needed to provide a better understanding of the materials and to optimize the joining processes for specific applications. 


\section{CHAPTER TWO: MATERIALS}

This chapter introduces the as-received materials used in this study and the properties and potential applications of these starting materials.

\section{1: Inconel 600}

Inconel 600 is the metal component used in this work, manufactured by Special Metals [28]. Inconel 600 is considered a high-performance Nickel-based alloy, wellsuited for extreme pressure and heat environments, as well as being corrosion resistant. It consists of an austenitic nickel-chromium-iron base solid solution strengthened by cold working [29]. Low concentrations of carbon, manganese, sulfur, copper, aluminum, thallium, cobalt, niobium, tantalum, and phosphorous are also present in this solid solution alloy. A solid solution is a mixture of two or more components in the solvent. It is a multi-component material whose crystal structure remains unchanged from the original crystal structure of the solvent. The high nickel concentration lowers the probability of the alloy corroding in many organic and inorganic compounds and also makes it resistant to chloride-ion stress-corrosion cracking [28]. The increased chromium content in the alloy, as compared to other metal alloys, improves the high temperature resistance to sulfur compounds as well as increasing resistance to oxidation at high temperatures or in corrosive solutions. The alloy also has excellent mechanical properties and presents the desirable combination of high strength and good workability. The Inconel 600 alloy does not suffer embrittlement after long exposure to high temperatures and shows stability of its mechanical properties. Service temperatures range from cryogenic to $1000{ }^{\circ} \mathrm{C}$. The composition of the Inconel 600 alloy used in this study is given in Table 2 . Some relevant properties of the Inconel 600 alloy are given in 
Table 3.

Table 2 The composition of the Inconel 600 superalloy used in this research study as received from Special Metals.

\begin{tabular}{|c|c|c|c|c|c|l|l|l|l|}
\hline Elements & $\mathbf{C}$ & $\mathbf{M n}$ & $\mathbf{F e}$ & $\mathbf{S}$ & $\mathbf{S i}$ & $\mathbf{C u}$ & $\mathbf{N i}$ & $\mathbf{C r}$ & $\mathbf{A l}$ \\
\hline Wt \% & 0.02 & 0.35 & 8.96 & 0.001 & 0.1 & 0.04 & 73.97 & 15.77 & 0.19 \\
\hline Elements & $\mathbf{T i}$ & $\mathbf{C o}$ & $\mathbf{N b}$ & $\mathbf{T a}$ & $\mathbf{P}$ & & & & \\
\hline Wt \% & 0.27 & 0.05 & 0.06 & 0.001 & 0.005 & & & & \\
\hline
\end{tabular}

Table 3 The Inconel 600 super alloy physical properties. All properties shown are from the CES EduPack database [26].

\begin{tabular}{|c|c|}
\hline Properties & Values \\
\hline Melting Point $\left({ }^{\circ} \mathrm{C}\right)$ & $1,360-1,420$ \\
\hline Service Temperature $\left({ }^{\circ} \mathrm{C}\right)$ & -273 to 982 \\
\hline CTE $\left(\mu\right.$ strain $\left./{ }^{\circ} \mathrm{C}\right)$ & $12-17$ \\
\hline Vickers Hardness $(\mathrm{HV})^{\text {Density }\left(\mathrm{g} / \mathrm{cm}^{3}\right)}$ & $135-190$ \\
\hline Young's Modulus $(\mathrm{GPa})$ & 8.4 \\
\hline Tensile Strength $(\mathrm{MPa})$ & $207-218$ \\
\hline Compressive Strength $(\mathrm{GPa})$ & $655-827$ \\
\hline Stress Corrosion Cracking & $56.5-62.4$ \\
\hline Corrosion Resistance to Inorganic and & Not Susceptible \\
\hline Organic Acids & Moderate \\
\hline Corrosion Resistance & Excellent \\
\hline Crystal Structure & FCC \\
\hline Bonding & Metallic \\
\hline
\end{tabular}

Because of its mechanical strength and corrosion resistance, Inconel 600 is used

extensively in the chemical industry [28]. Applications include heaters, stills, bubble towers and condensers for processing of fatty acids; evaporator tubes, tube sheets and flaking trays for the manufacture of sodium sulfide; and equipment for handling acidic solvents in the manufacture of paper pulp. In addition, the alloy's strength and oxidation resistance at high temperatures make it useful for many applications in the heat-treating industry. It is used for retorts, muggles, roller hearths and other furnace components and for heat-treating baskets and trays. In the aeronautical field, Inconel 600 is used for 
variety of engine and airframe components which must withstand high temperatures [28].

Also, the alloy is a standard material for the construction of nuclear reactors. It has excellent resistance to corrosion by high purity water, and it is resistant to chloride-ion stress-corrosion cracking in reactor water systems. Because of these numerous advantages, the Inconel 600 alloy is a good candidate for the diffusion bonding to reaction-bonded $(\mathrm{RB})-\mathrm{SiC}$ for the next generation of high temperature applications.

In addition, the following Figure 11 shows two different Ashby plots showing how Inconel 600 properties considered for the application of interest in this investigation (e.g. mechanical properties vs maximum service temperature capacity) compare to other potential metals used for high temperature applications.

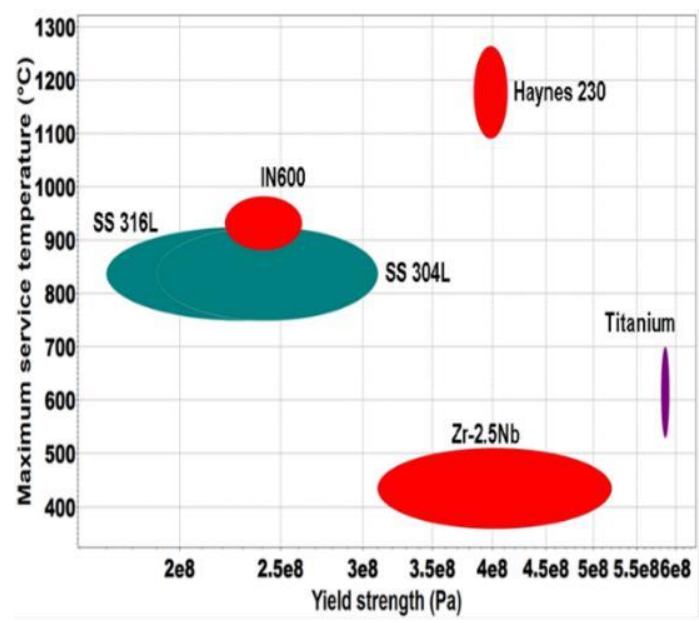

(a)

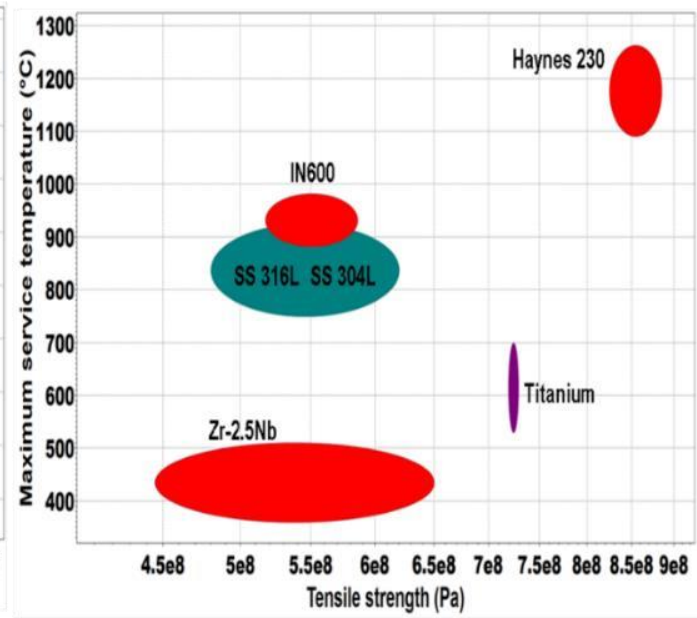

(b)

Figure 11 Ashby plots showing how Inconel 600 compares to other commonly used high performance metals if plotting (a) maximum service temperature versus yield strength and (b) maximum service temperature versus tensile strength [26].

Figure 12 shows how Inconel 600 prize compares to other potential metals commonly used for applications of similar characteristics. Although not the cheapest when comparing quality/prize Inconel 600 is a well suited material. 


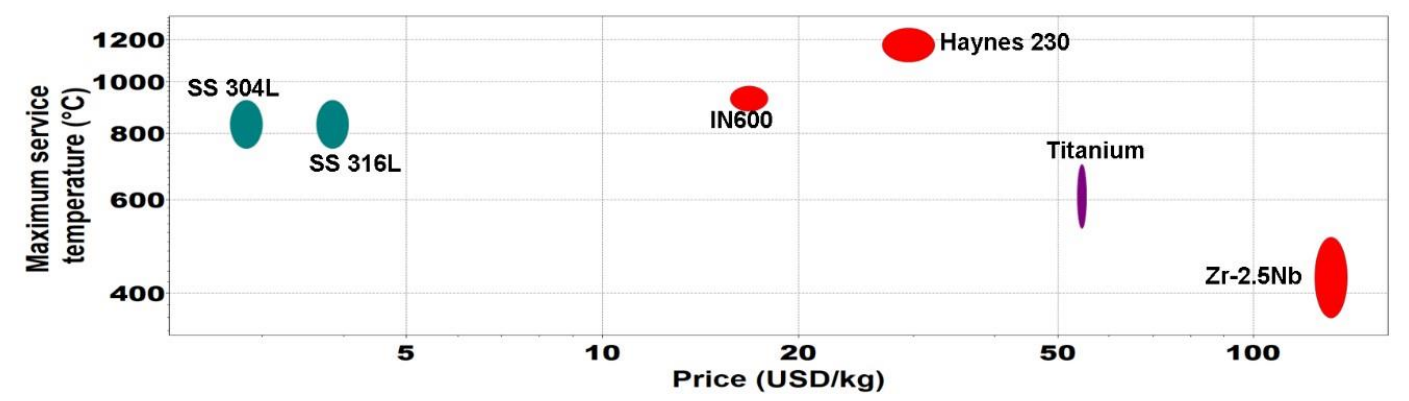

Figure 12 Ashby plot showing the comparison of all well suited metals for high temperature applications by plotting maximum service temperature versus price [26].

\section{2: Silicon Carbide (SiC)}

SiC pellets were purchased from Coorstek Inc. [30]. This material is a reactionbonded silicon carbide ( $\mathrm{RB}-\mathrm{SiC})$, sometimes referred to as siliconized silicon carbide ( $\mathrm{SiSiC})$. The $\mathrm{SiSiC}$ fabrication process usually requires either a mixture of $\mathrm{SiC}$ and carbon grains, or a preformed carbon matrix infiltrated with molten silicon ( $\mathrm{Si}$ ) or siliconrefractory metal alloys. This second technique, called liquid silicon infiltration (LSI), relies on chemical interactions between the filler and the base matrix material [31, 32]. The process involves temperatures exceeding the melting point of $\mathrm{Si}, 1,414{ }^{\circ} \mathrm{C}$. The liquid silicon component reacts with the carbon, forming silicon carbide ( $\mathrm{SiC}$ ) according to reaction 2.1 :

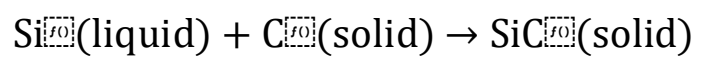

In general, at least $5 \%$ of residual free silicon is left in the $\mathrm{SiC}$ matrix. Therefore, a more representative reaction 2.2 looks like:

$$
\text { 2Sifo:(liquid) }+ \text { Co: (solid) } \rightarrow \text { SiCo:(solid) }+ \text { Sifo:(solid) }
$$


The infiltration process results in a material with a unique combination of mechanical, thermal, and electrical properties, which can be tuned to the application. The physical properties of reaction bonded SiC are shown in Table 4.

Table 4 SiC characteristics. All properties shown are from the CES EduPack database [26].

\begin{tabular}{|c|c|}
\hline Properties & Values \\
\hline Melting Point $\left({ }^{\circ} \mathrm{C}\right)$ & $2,147-2,247$ \\
\hline Service Temperature $\left({ }^{\circ} \mathrm{C}\right)$ & -273 to 1430 \\
\hline CTE $\left(\mu\right.$ strain $\left./{ }^{\circ} \mathrm{C}\right)$ & $2.3-4.3$ \\
\hline Vickers Hardness $(\mathrm{HV})$ & $1,900-3,150$ \\
\hline Density $\left(\mathrm{g} / \mathrm{cm}^{3}\right)$ & 3.15 \\
\hline Young's Modulus $(\mathrm{GPa})$ & $400-420$ \\
\hline Tensile Strength $(\mathrm{MPa})$ & $191-326$ \\
\hline Compressive Strength $(\mathrm{MPa})$ & $1.9-2.1$ \\
\hline Corrosion Resistance & Excellent \\
\hline Crystal Structure & Hexagonal \\
\hline Bonding & Covalent \\
\hline
\end{tabular}

Silicon carbide ( $\mathrm{SiC}$ ) is one of the most promising high-temperature materials due

to its excellent properties: superior high-temperature strength, high thermal shock resistance, good corrosion and acid resistance [15]. It is a promising structural electrical and thermal material for use in advanced heat engines and in the nuclear industry for high temperature applications [15]. However, $\mathrm{SiC}$ is difficult to form into large and complex shapes, requiring the joining of $\mathrm{SiC}$ to itself or to metals [11]. SiC/metal composite structures offer significant performance advantages over single-component ceramic or metallic structures [33].

$\mathrm{SiC}$ is used in high-temperature electronic devices such as aircraft and automotive engine sensors, jet engine ignition systems, transmitters for deep well drilling, and a number of industrial process measurement and control systems. The use of SiC-based distributed smart electro-mechanical controls which are capable of harsh-ambient 
operation will enable substantial jet-aircraft weight savings, reduced maintenance, reduced pollution, higher fuel efficiency, higher thermal efficiency, and increased operational reliability. Reaction bonded $\mathrm{SiC}$ is also used as a structural material in such devices as heat exchangers.

In addition, Figure 13 shows two different Ashby plots showing how SiC properties considered for the application of interest in this investigation (e.g. mechanical properties vs maximum service temperature capacity) compare to other suitable ceramics used for high temperature applications. Also, Figure 14 shows how prize was a driven force within the material selection process, specially comparing Aluminum Nitride properties to $\mathrm{SiC}$ as well as in prize.

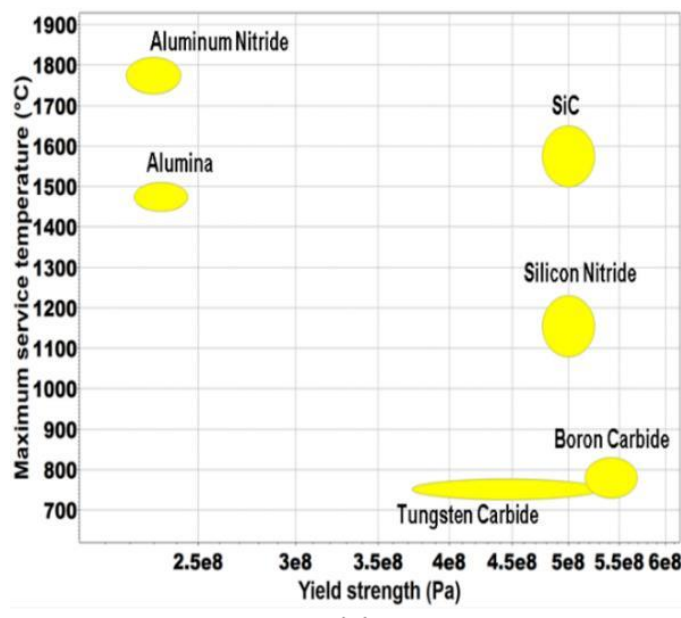

(a)

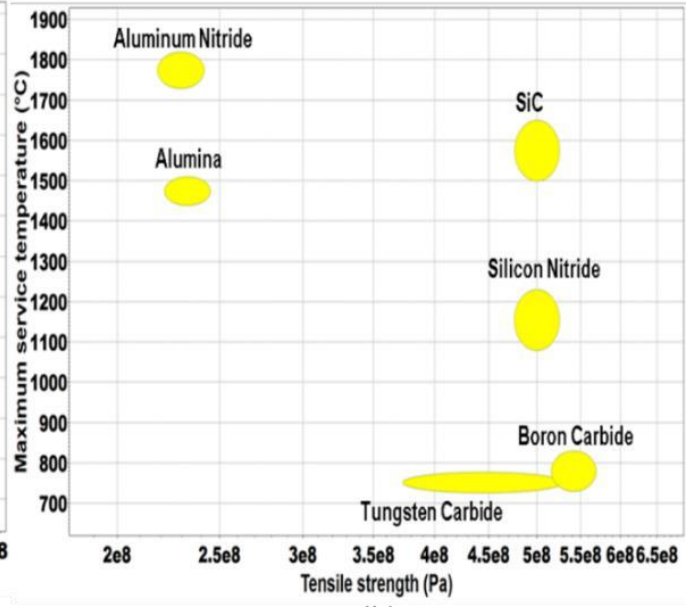

(b)

Figure 13 Ashby plots showing how SiC compares to other commonly used high temperature ceramics if plotting (a) maximum service temperature versus yield strength and (b) maximum service temperature versus tensile strength [26]. 


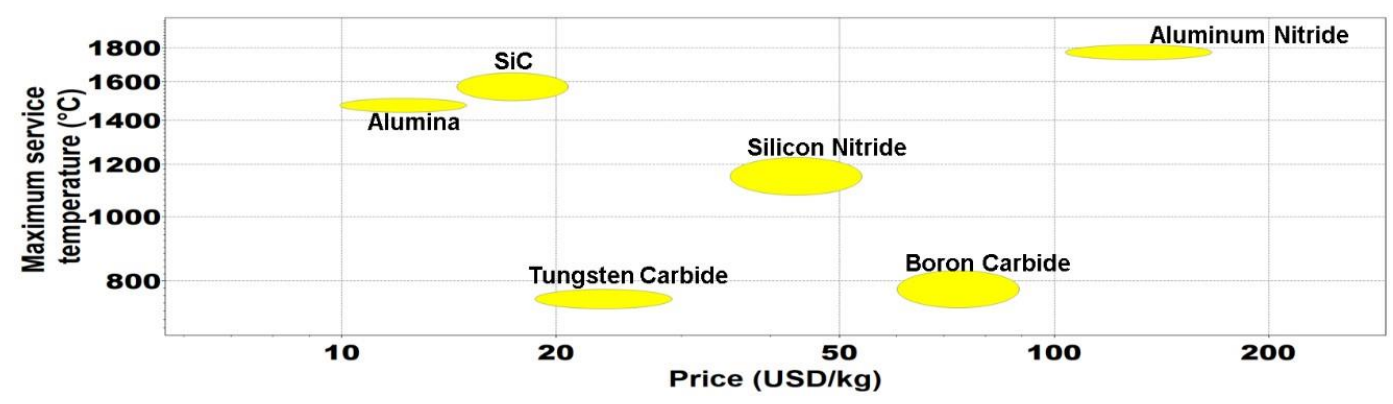

Figure 14 Ashby plot showing the comparison of all well suited high performance ceramics for high temperature applications by plotting their maximum service temperature versus price [26].

\section{3: Interlayer Materials}

99.9\% silver (Ag) was one of the two interlayers used in this work. It was

purchased from RioGrande [34]. One reason that Ag was chosen as a suitable candidate for this work is because it does not react with silicon to form silicides [8]. Silicides are typically brittle and degrade the interface mechanical properties of the formed SiC/Inconel 600 joints. In addition, the ductility of Ag helps to accommodate the CTE mismatch between the ceramic-metal components. Table 5 shows the relevant material properties of $\mathrm{Ag}$.

Table 5 Silver alloy characteristics. All properties are from the CES EduPack database [26].

\begin{tabular}{|c|c|}
\hline Properties & Values \\
\hline Melting Point $\left({ }^{\circ} \mathrm{C}\right)$ & $957-967$ \\
\hline Service Temperature $\left({ }^{\circ} \mathrm{C}\right)$ & -273 to 190 \\
\hline CTE $\left(\mu\right.$ strain $\left./{ }^{\circ} \mathrm{C}\right)$ & $19-22$ \\
\hline Vickers Hardness $(\mathrm{HV})$ & $25-35$ \\
\hline Density $\left(\mathrm{g} / \mathrm{cm}^{3}\right)$ & 10.5 \\
\hline Young's Modulus $(\mathrm{GPa})$ & $70-74$ \\
\hline Tensile Strength $(\mathrm{MPa})$ & $110-175$ \\
\hline Stress Corrosion Cracking & Not susceptible \\
\hline Crystal Structure & FCC \\
\hline Bonding & Metallic \\
\hline
\end{tabular}

$\mathrm{Ag}_{75} \mathrm{Pd}_{25}$ was the second interlayer used in this work. The interlayer material was purchased from ESPI Metals [35]. The addition of $25 \mathrm{wt} \%$ palladium to silver improves 
relevant properties such as the operating temperature, the yield strength and Young's modulus. The addition of Pd raises the melting temperature of the interlayer, therefore increasing the service temperature of the fabricated joint. It also results in increased stiffness and decreased ductility compared to pure $\mathrm{Ag}$, which could improve the performance of the interlayer when high pressures are applied. Table 6 lists some of the estimated properties values.

Table 6 Silver-Palladium 25\% alloying characteristics. Properties are measured using the rule of mixtures as explained below [26].

\begin{tabular}{|c|c|}
\hline Properties & Values \\
\hline Melting Point ${ }^{\circ} \mathrm{C}$ & $1,063-1,110$ \\
\hline Service Temperature ${ }^{\circ} \mathrm{C}$ & -273 to 243 \\
\hline CTE $\left(\right.$ strain $\left./{ }^{\circ} \mathrm{C}\right)$ & $17-20$ \\
\hline Vickers Hardness $(\mathrm{HV})$ & $28.5-42.5$ \\
\hline Density $\left(\mathrm{g} / \mathrm{cm}^{3}\right)$ & 10.85 \\
\hline Young's Modulus $(\mathrm{GPa})$ & $77.5-82$ \\
\hline Tensile Strength $(\mathrm{MPa})$ & $143-221$ \\
\hline Stress Corrosion Cracking & Not susceptible \\
\hline Crystal Structure & FCC \\
\hline Bonding & Metallic \\
\hline
\end{tabular}

All the properties of the silver-palladium alloy were estimated using the rule of mixtures. In general, the weighted mean can be used to predict various properties of the solid solution Ag-Pd alloy. The palladium was considered the distributed phase, while the silver was considered to be the matrix for the equations below. Generally, the rule of mixtures has two limits, depending on the alignment of the distributed phase (parallel or perpendicular) to the applied load. The properties were presented as a range of values from the average values calculated from the two upper and lower limits equations. The following equation is a general equation describing the upper limit property [18]: 


$$
\mathrm{P}_{\text {upper }}=\mathrm{fPPd}+(1-\mathrm{f}) \mathrm{PAg}_{\mathrm{Ag}}
$$

The following equation is the general equation describing the lower limit property [18]:

$$
\mathrm{P}_{\text {lower }}=\left(\frac{\mathrm{f}}{\mathrm{P}_{\mathrm{Pd}}}+\frac{1-\mathrm{f}}{\mathrm{P}_{A g}}\right)^{-1}
$$

Note: Pupper $\neq$ Plower

The variables found within the equations correspond to the following $[18] \mathrm{f}=\frac{\mathrm{V}_{\mathrm{Pd}}}{\mathrm{V}_{P d}+\mathrm{V}_{\mathrm{Ag}}}$ is the volume fraction of the fibers or phase material, $\mathrm{P}_{\mathrm{Pd}}$ is the property value from the $\mathrm{Pd}$, and $\mathrm{P}_{\mathrm{Ag}}$ is the property value from the $\mathrm{Ag}$.

The rule of mixtures was chosen as the technique to estimate the properties of the interlayer alloy used in this work due to the lack of literature data on this Ag-Pd $25 \%$ alloy specific composition, specially at elevated temperatures.

In summary, this section presented the chosen materials for this investigation and listed the important properties of each material relative to this study. The next section describes the experimental processes for fabricating and characterizing the joints.

\section{CHAPTER THREE: EXPERIMENTAL PROCEDURE}

This chapter describes the process developed to prepare the starting materials for bonding, introduces the equipment used to form the ceramic/metal joints, and provides an overview of the theoretical calculations used to understand this system. In addition, the sample preparation for the various characterization techniques is explained. Scanning electron microscopy (SEM), energy dispersive spectroscopy (EDS) and X-Ray diffraction 
(XRD) were used to characterize the interfacial reactions. Finally, this chapter details the investigation of the mechanical properties of the ceramic/metal joints.

Diffusion bonding experiments were designed using a multiple process parameter test matrix to study the effects of bonding temperature, pressure, and time on the Inconel 600 to $\mathrm{SiC}$ interfacial microstructure, the formation of solid-state reaction phases, and the bonding integrity. Figure 15 is a schematic representation of the joining process.

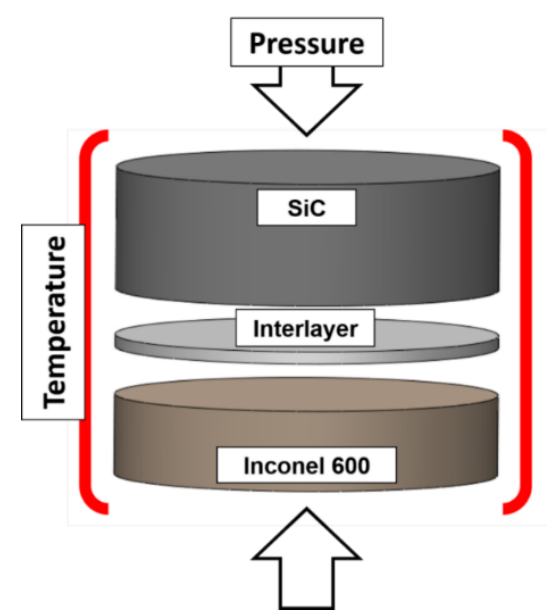

Figure 15 Schematic of the sample configuration with the silicon carbide (SiC) and Inconel 600 main components and the interlayer as Ag or Ag75Pd25.

The test matrix was designed to study the impact of various process parameters and their interaction on the interfacial morphology of the joints. The combinations studied were two different temperatures, pressures, and times. In addition to these parameters, two interlayers ( $\mathrm{Ag}$ and $\left.\mathrm{Ag}_{75} \mathrm{Pd}_{25}\right)$ were investigated. The bonding experiments were carried out using the process parameter combinations, shown in Table 7, for both interlayer systems. The matrix was repeated for the second interlayer system.

Table 7 Process parameter matrix for samples

\begin{tabular}{|c|c|c|c|}
\hline Test & Temperature $\left({ }^{\circ} \mathrm{C}\right)$ & Pressure $(\mathrm{MPa})$ & Time $(\mathrm{min})$ \\
\hline 1 & 900 & 1 & 30 \\
\hline 2 & 900 & 10 & 30 \\
\hline
\end{tabular}




\begin{tabular}{|c|c|c|c|}
\hline 3 & 930 & 10 & 30 \\
\hline 4 & 930 & 10 & 180 \\
\hline
\end{tabular}

By investigating these four process combinations, the effect of temperature, pressure and time were studied individually. The pressure effect was studied when comparing interfacial microstructure results from test 1 and 2 . The temperature effect was studied when comparing interfacial microstructure results from test 2 and 3. Finally, the time effect was investigated by comparing interfacial microstructure results from test 3 and 4.

\section{1: Sample Preparation before bonding}

The components used in the diffusion bonding experiments investigated in this work were prepared as follows:

1. The Inconel 600 pellets were cut via electrical discharge machining (EDM) from a sheet of thickness $2.7 \mathrm{~mm} \pm 0.13 \mathrm{~mm}$ to a diameter of $8 \mathrm{~mm}$. Then, the pellets were polished using the lapping and polishing fixture (South Bay Technology Model 155) at 600-grit, 800-grit, and 1,200-grit SiC paper to maintain parallel faces and a uniform thickness for even pressure distribution. Pellets were then polished using the $9-\mu \mathrm{m}$ and $3-\mu \mathrm{m}$ water-based diamond suspensions on woven or napped fiber pads. The average thickness of the finished pellets was $2.5 \mathrm{~mm} \pm .2$ $\mathrm{mm}$ with a degree of parallelism of $0.005 \mathrm{~mm}$.

2. Two sets (1) and (2) of $8 \mathrm{~mm}$ diameter $\mathrm{SiC}$ rods were received. Set (1) was sliced using a slow speed saw and then ground to a thickness of $3 \mathrm{~mm} \pm 0.2 \mathrm{~mm}$ with a 320-grit wheel by Advanced Ceramic Technology in California. The pellets were further polished using a vibratory polisher for 14 hours with the silica solution. When more pellets were needed to validate the experiments, $\mathrm{SiC}$ rods were cut 
and prepared in-house. Set (2) of SiC pellets were sliced with a slow speed saw and polished using the lapping fixture to assure parallel faces. First, they were polished using a 320-grit metal plate, then by $\mathrm{SiC}$ paper at 600, 800, and 1,200 grit. Lastly, the pellets were polished using the $9-\mu \mathrm{m}$ water-based diamond suspension on woven or napped fiber pads.

3. The $0.254 \mathrm{~mm}$ thick, silver interlayer was cut by hand to the $8 \mathrm{~mm}$ diameter. The silver-palladium alloy was cut using EDM to the exact diameter. Both materials were polished with the $9-\mu \mathrm{m}$ and $3-\mu \mathrm{m}$ water-based diamond suspensions on woven or napped fiber pads before joining.

Following polishing, pellets were rinsed with isopropanol (IPA), deionized (DI) water, and ethanol sequentially. Finally, a micrometer was used to measure the thickness around the perimeter of each pellet in at least five locations to assure that the surfaces were flat and the pressure applied during the bonding test would be distributed homogeneously.

\section{2: Joining Procedure}

The overall set up used to fabricate the Inconel 600/interlayer/SiC joints is shown in Figure 16. Figure 16 (b) shows the test system (MTS), model 318.10, mechanical test frame. Figure 16 (a) shows the FlexTest SE Plus device that couples the MTS frame to the computer and allows for the monitoring and programming of the processing conditions. The FlexTest facilitated the customization of the procedures and generated test reports with time, temperature, and pressure data for post-testing analysis. The high axial and lateral stiffness of the MTS provided uniform and precise application of pressure, while the crosshead mounted load cell allowed for accurate force control. The 
load applied to the sample during the diffusion bonding test was monitored by the system load cell with a maximum load capability of $100 \mathrm{kN}$. An MTS Furnace, Model 653.04, built specifically for high temperature testing was used for the bonding experiments. Three pairs of silicon carbide heating elements were arranged in three zones. Insulation plates between the elements offered reliable zone separation, and pre-cut insulation helped to reduce heat loss. The furnace was capable of attaining temperatures of up to $1,400{ }^{\circ} \mathrm{C}$. The internal (hot zone) furnace dimensions were height $(185 \mathrm{~mm})$, width $(62.5$ $\mathrm{mm})$ and depth $(62.5 \mathrm{~mm})$. The Model 409.83 temperature controller paired with the furnace is shown in Figure 16. This furnace was an ideal choice for these experiments because achieving a low thermal gradient on these joints was required for the success of the experiments. The configuration of the device for these joining experiments is shown in Figure 17. Inconel 600 push rods which maintain strength at the testing temperatures were machined to fit into a self-aligning compression test fixture. 


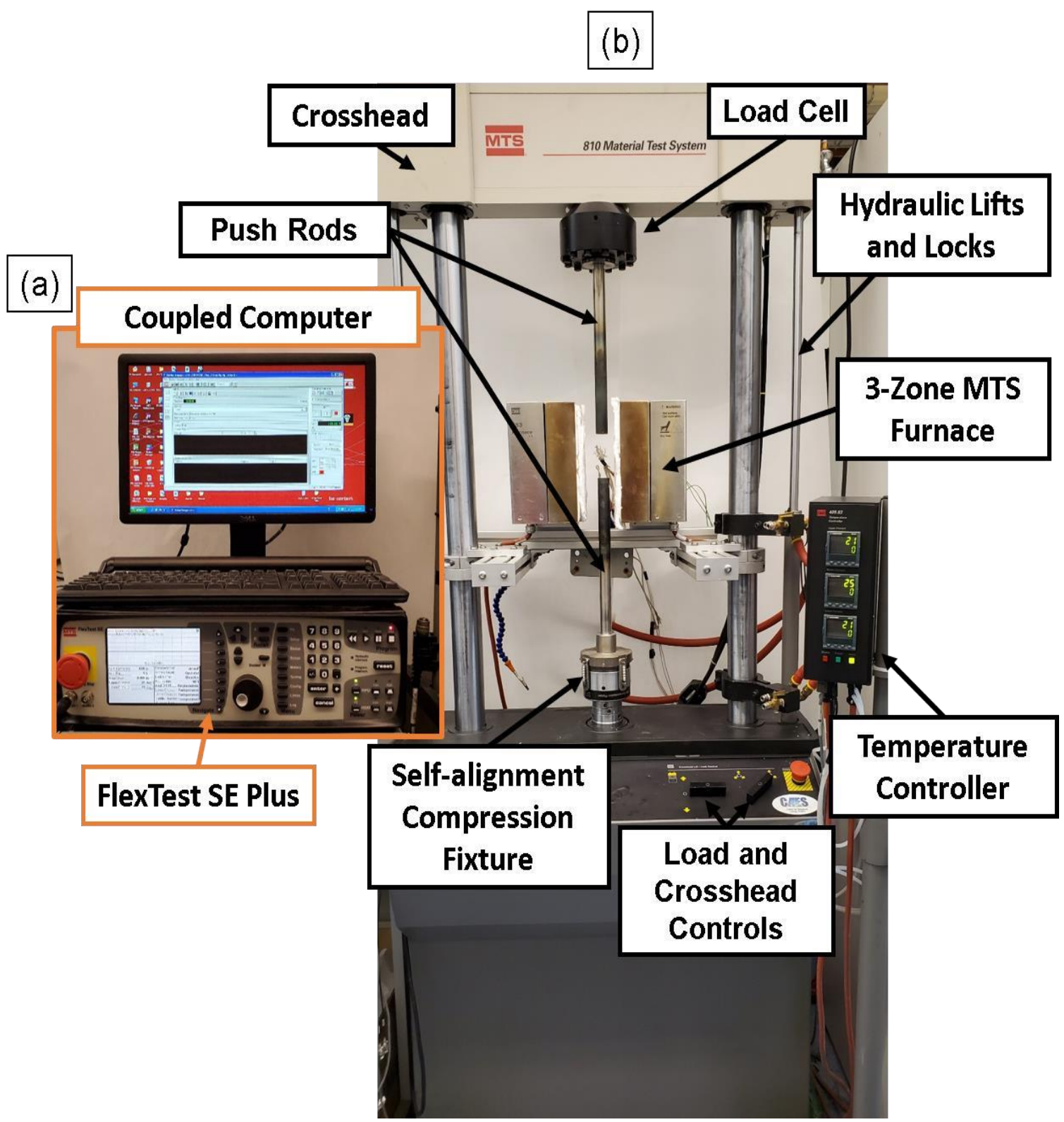

Figure 16 Image (a) shows the FlexTest SE Plus coupled with the computer controlling the MTS. Image (b) shows the specific set up for Material Test System (MTS) mechanical test frame used for the formation of SiC/Inconel 600 joints with $\mathrm{Ag}$ and $\mathrm{Ag}_{75} \mathbf{P d}_{25}$ as interlayers. 


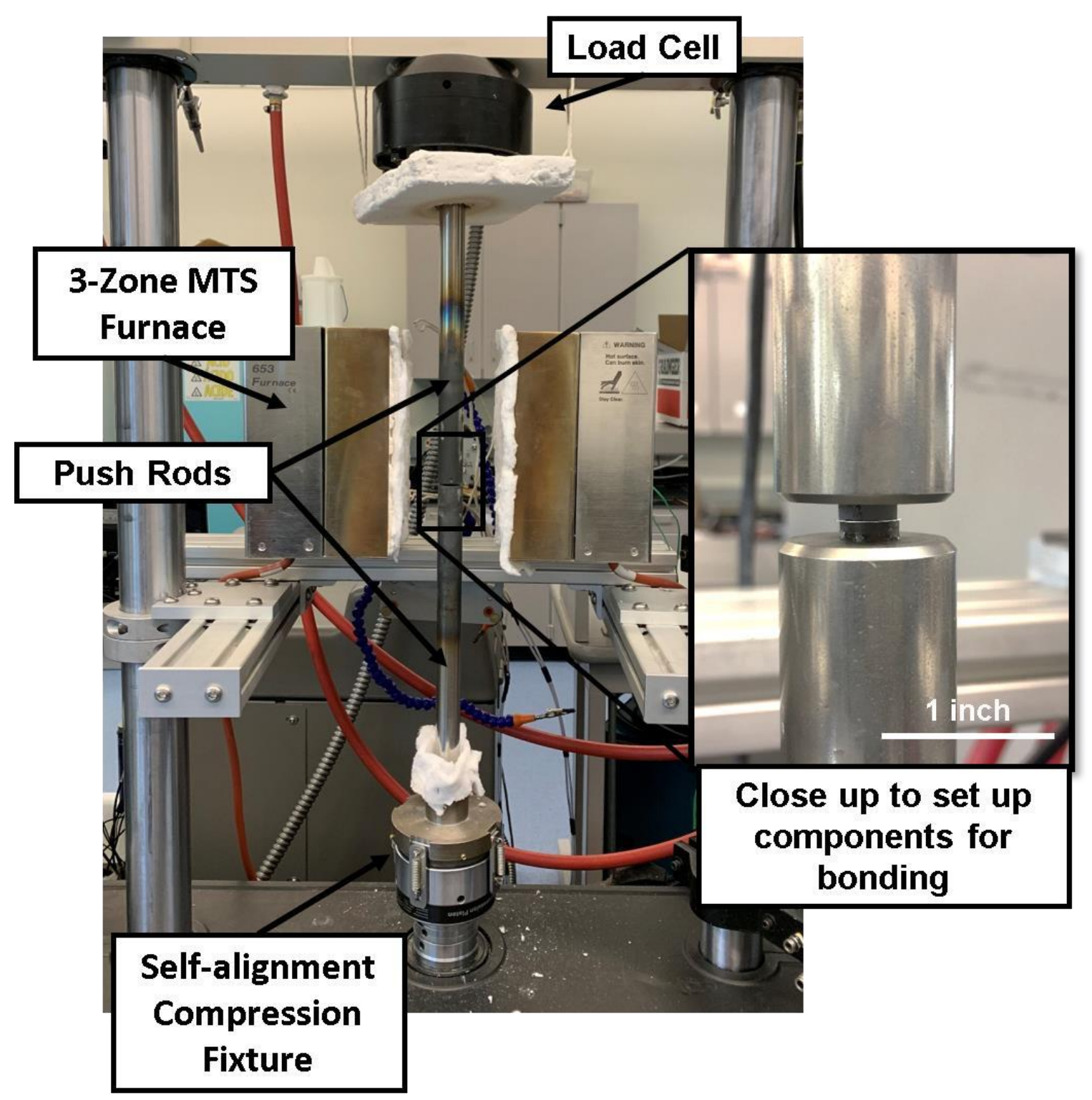

Figure 17 Experimental set up of the MTS mechanical test frame for diffusion bonding experiments showing a close up of a sample before bonding.

Diffusion bonding is a thermally activated process and is usually performed at a temperature range between $60-95 \%$ of the interlayer melting point $[3,18,21]$. For this reason, the maximum temperature investigated was $930{ }^{\circ} \mathrm{C}$, which is $\approx 07 \%$ of the melting temperature of the Ag interlayer. The monitored temperature was validated with a calibrated external K-type thermocouple placed close to the joint. The top and bottom K-type thermocouple wires were calibrated with an external calibrated TC as point of 
reference. They were positioned in contact with the push rods to assure that temperature was homogeneous throughout. Figure 18 shows the set up of the different K-type TCs around the sample during bonding.

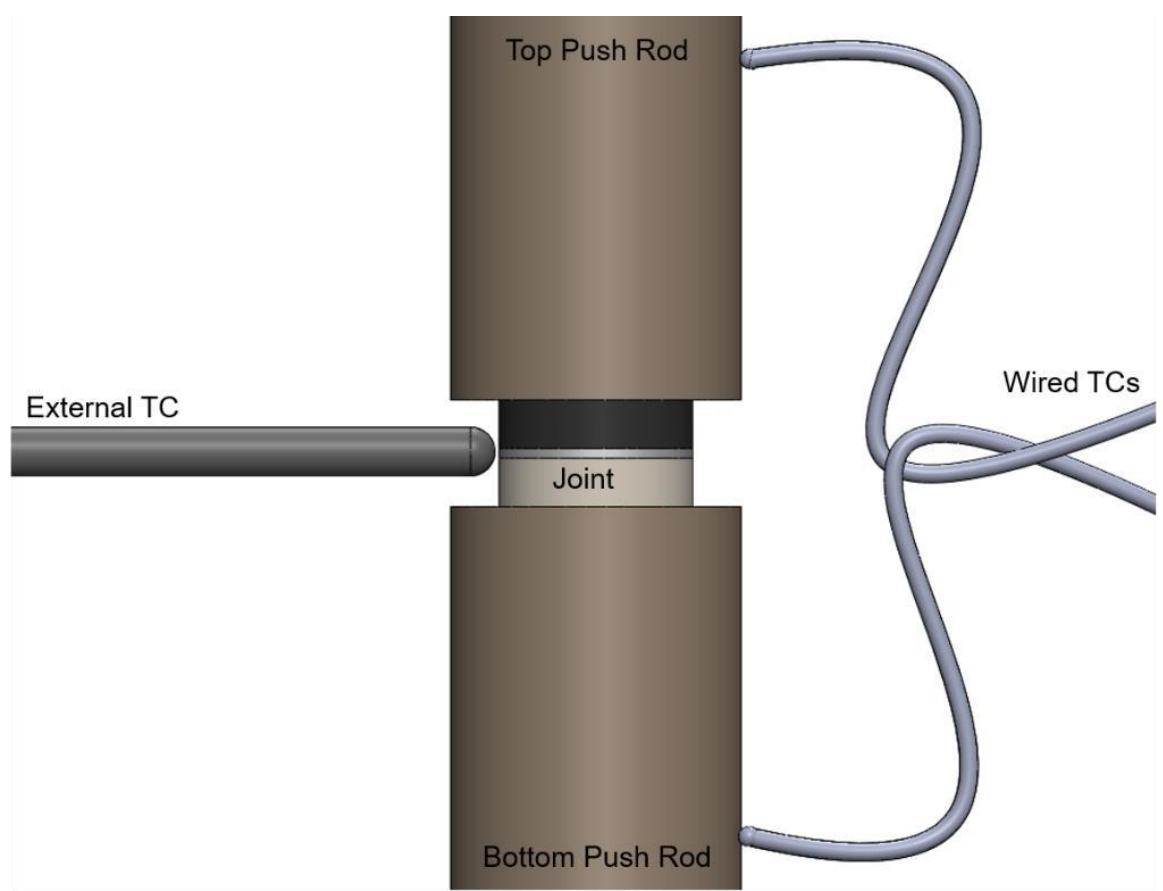

Figure 18 Schematic image showing the thermocouple set up to monitor the insitu temperature during bonding experiments.

Figure 19 shows the two extremes of the diffusion bonding profiles used to fabricate the $\mathrm{SiC} / \mathrm{Inconel} 600$ joints with $\mathrm{Ag}$ or $\mathrm{Ag}_{75} \mathrm{Pd}_{25}$ as the interlayer. The heating and cooling rate used for all experiments was $10{ }^{\circ} \mathrm{C} / \mathrm{min}$, reaching $900-930{ }^{\circ} \mathrm{C}$ in 90 minutes. Once at temperature, pressure was applied and held for the specified diffusion bonding time of either 30 or 180 minutes. Once time was expired, the pressure was released and the bonding experiment was cooled down to room temperature. Temperature was not recorded for the entire cool down period, as shown in Figure 19. However, samples were left to cool down to room temperature before being taken out of the furnace. 
(a)

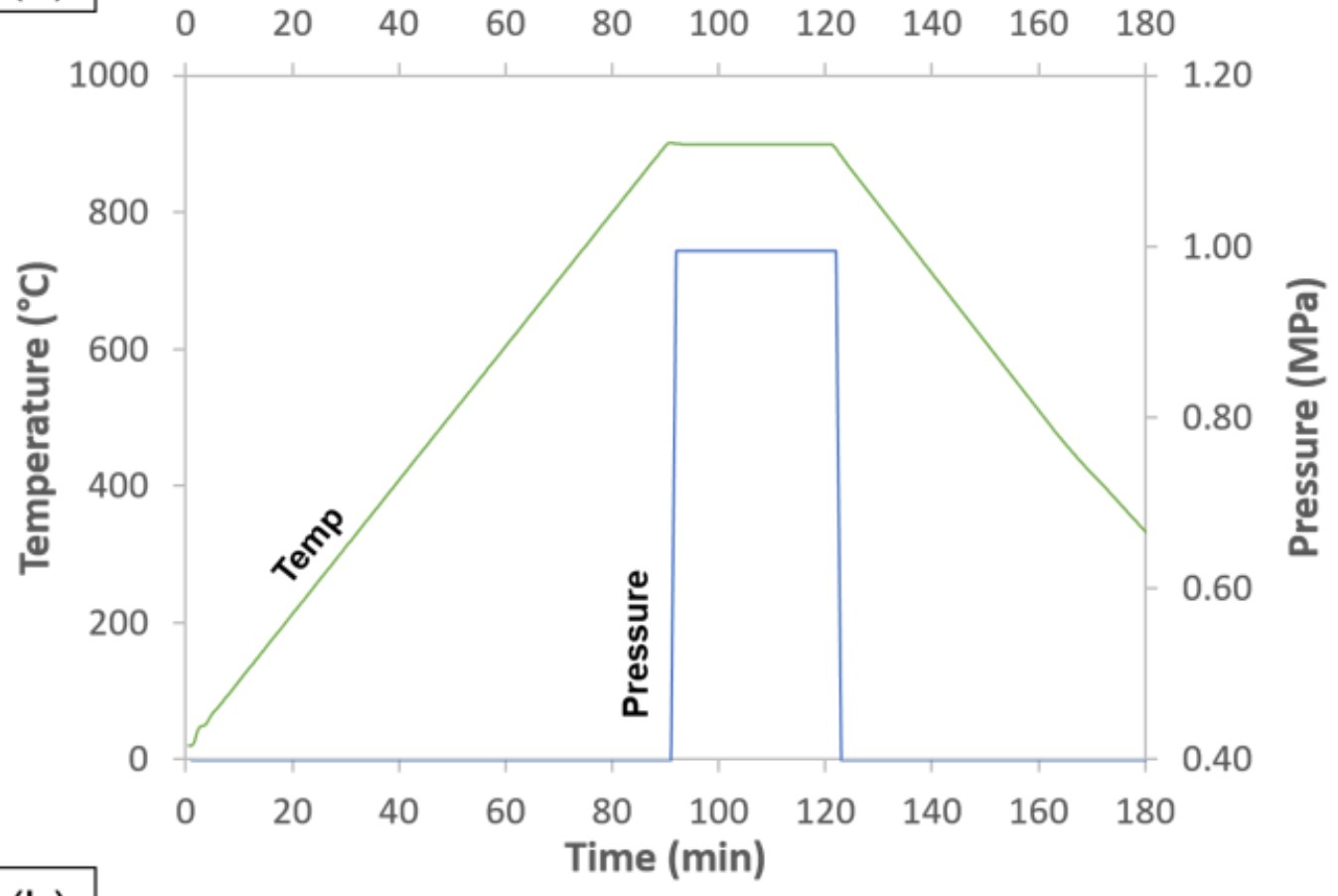

(b)

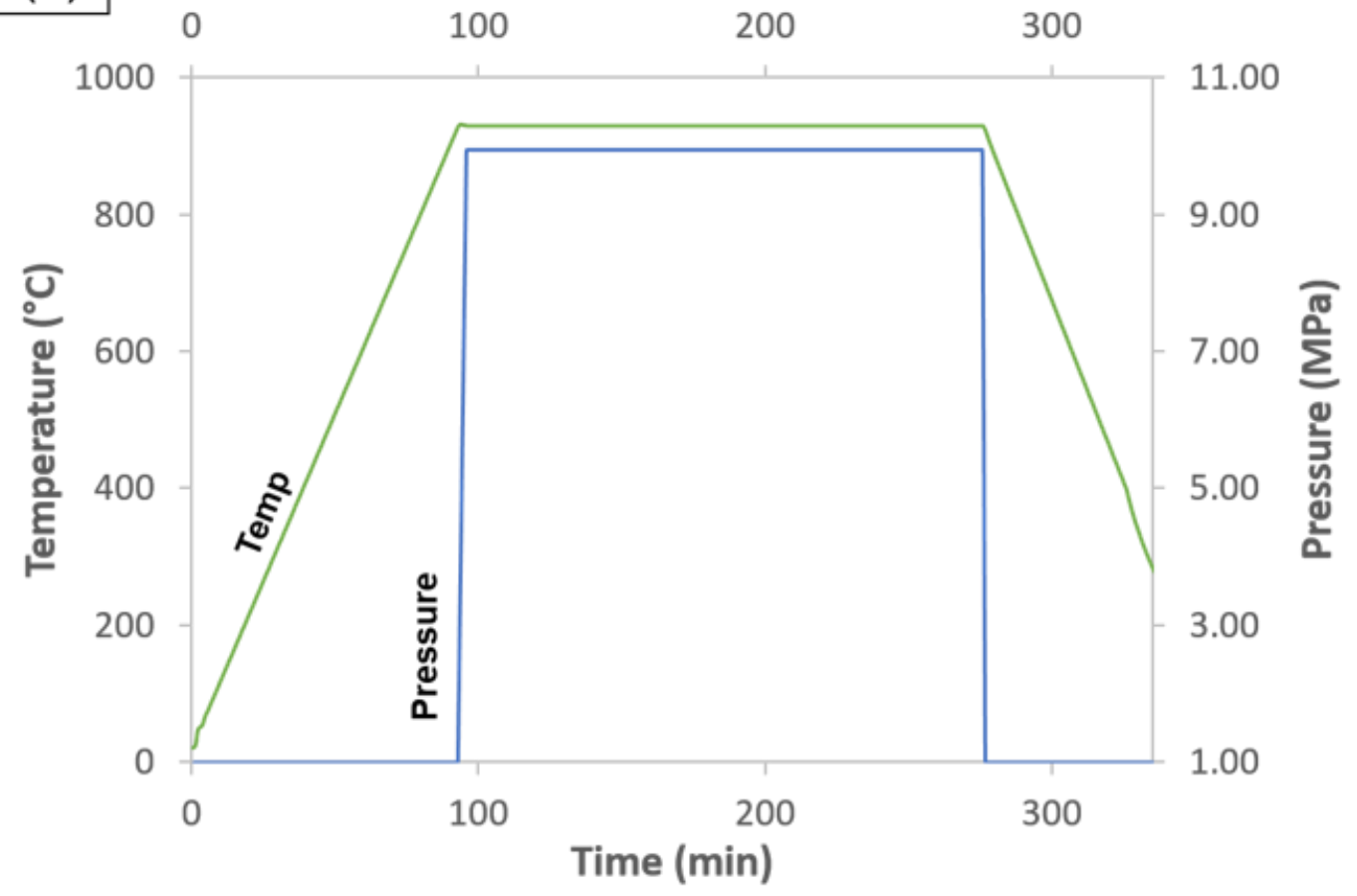

Figure 19 Diffusion bonding profiles showing temperature profile and applied pressure during formation of joint (a) at $900{ }^{\circ} \mathrm{C}$ for 30 minutes under $1 \mathrm{MPa}$ and (b) at $930{ }^{\circ} \mathrm{C}$ for 180 minutes under $10 \mathrm{MPa}$. Green lines correspond to the actual measured temperature data. Blue lines correspond to the programmed pressure parameter. 


\section{3: Sample Characterization}

After joining, samples were prepared for analysis by using SEM/EDS and XRD. In order to investigate the composition and morphology of the SiC/Inconel 600 interfaces, transverse sections of each joint were cut using a low-speed diamond cut-off wheel operated with an oil coolant. The mounting and cutting process is shown in Figure 20. Before cross sectioning the joints, the specimens were mounted in a two-part epoxy resin. After the joints were cross sectioned, one half of each was ground with SiC paper to 1200 grit on a polishing wheel using a rotation speed of $250 \mathrm{rpm}$. The polishing continued with diamond suspension in water of $9 \mu \mathrm{m}$ and $3 \mu \mathrm{m}$ to provide a final finish.

The samples were then rinsed with Isopropanol, DI water, and Ethanol sequentially. During the Ethanol rinse, they were also ultrasonicated to assure full cleanliness.

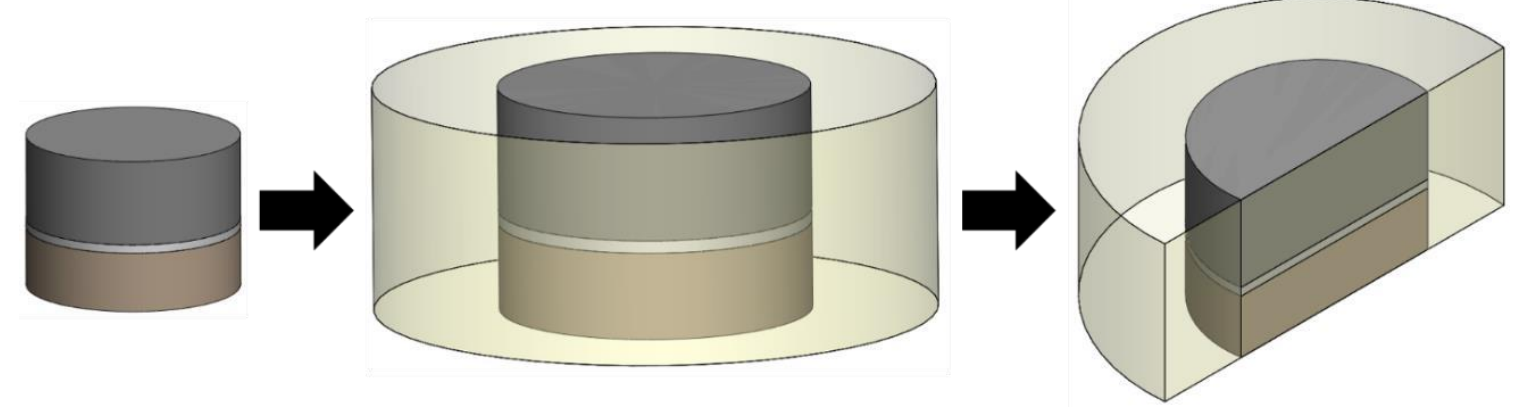

Figure 20 Schematic sequence of the preparation of the formed joints to polish them and prepare for characterization.

\subsection{1: Microstructural Characterization}

The interfaces were examined on the polished, cross-sectioned samples using a Hitachi S-3400N-II Analytical Scanning Electron Microscope (SEM) shown in Figure 21. The resolution of the equipment is $3 \mathrm{~nm}(\mathrm{SEI})$ and $4 \mathrm{~nm}(\mathrm{BSI})$. The Energy dispersive spectroscopy (EDS) detector has a resolution $\leq 136 \mathrm{eV}$ at MnKa FWHM [36]. EDS was used to perform chemical analysis on the samples and identify new phases forming as 
result of the diffusion and subsequent chemical reactions at the interface. The thickness of the resulting interfaces was measured directly from secondary back-scattered electron images using ImageJ software.

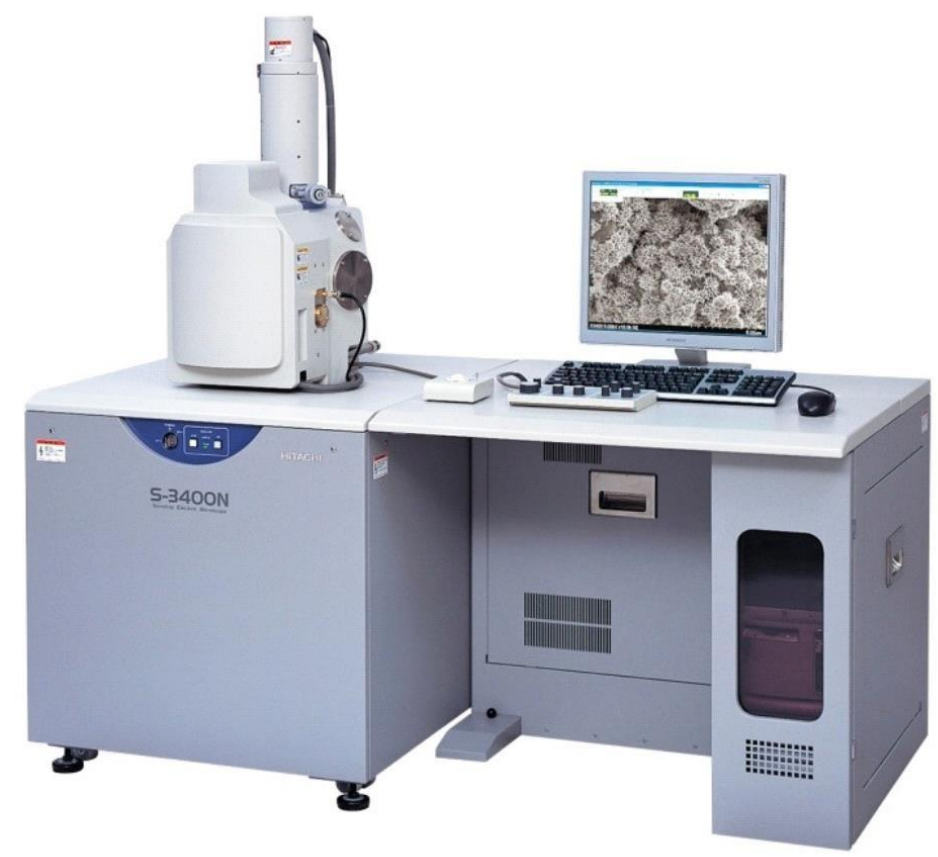

Figure 21 Hitachi S-3400N-II Analytical Scanning Electron Microscope (SEM) for characterization from the Boise State Center for Materials Characterization at Boise State University [37].

\subsection{2: X-Ray Diffractometry}

X-ray diffraction was carried out using the Rigaku MiniFlex 600 bench-top X-ray diffractometer with a one dimensional ultra-high-speed D/teX detector. The angular range between 10 to 140 of $2 \theta$ was scanned with an angular velocity of $4 \%$ min, using an accelerating voltage of $40 \mathrm{kV}$ and a current of $15 \mathrm{~mA}$. Measurements were performed on the starting materials and the fracture surfaces. The phases present in the samples were identified by comparing the experimental diffraction patterns with XRD reference patterns available in the ICSD database. Figure 22 shows the Rigaku Miniflex XRD. 


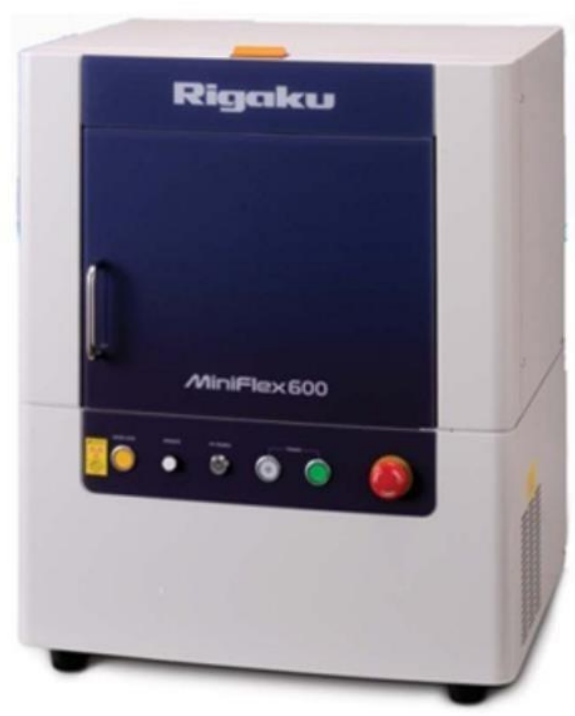

Figure 22 Rigaku Miniflex 600 bench-top X-ray diffractometer. The tool has a $600 \mathrm{~W}$ generator with copper source and is capable of a $2 \theta$ scan range of +2 to +145 $\circ$. It is capable of very quick scans because of the One-dimensional D/teX Ultrahighspeed detector. From the Boise State Center for Materials Characterization at Boise State University [38].

\section{4: Mechanical Evaluation}

The mechanical properties of the interfacial solid-state phases were investigated in order to determine the bonding strength and the weakest region that induced failure, as well as to validate the reaction regions that formed during the diffusion bonding.

\subsection{1: Tensile Test Evaluation}

Bond strength was measured by tensile testing at room temperature. A Shimadzu EZ-LX test frame was used to carry out the experiments. The load cell maximum force was $500 \mathrm{~N}$ and a sensitivity of $10.0 \%$ was used for the test procedure. A self-aligning fixture was designed to accommodate the specific geometry of the joints and to ensure a homogeneous uniaxial tensile load. Figure 23 shows the fixture designed to perform tensile tests on the joints formed in this work. A cyanoacrylate glue with a tensile strength of 2600 psi, was used to attach the joints to the Stainless 316 tensile rods. The glue was 
left to dry for 60 minutes before testing. The crosshead rate used for testing was

$1 \mathrm{~mm} / \mathrm{sec}$. Once the joints were tested, the rods were soaked in an acetone solution to let the cyanoacrylate glue dissolve away. The rods were not fully submerged to preserve the sample fracture surface for further characterization.

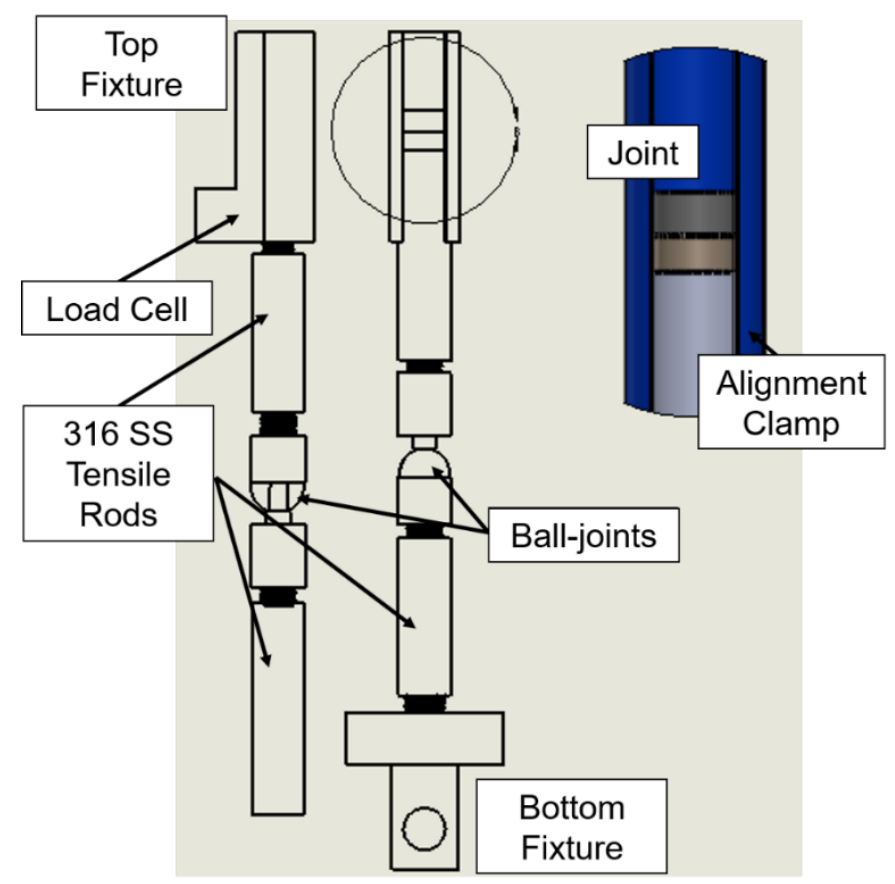

Figure 23 Tensile test fixture designed to conduct testing to investigate the bond strength for the formed $\mathrm{SiC} / \mathrm{Inconel} 600$ joints with $\mathrm{Ag}$ and $\mathrm{Ag}_{75} \mathrm{Pd}_{25}$ as the interlayer.

\subsection{2: Microhardness evaluation}

Microhardness was measured to determine the difference in hardness between the starting materials and the solid-state reaction phases that formed at the interface from the bonding process. The hardness was obtained using the LECO LM247AT micro-hardness tester at the Center for Advanced Energy Studies (CAES). A $200 \mathrm{~N}$ load was used for indents in the interlayer and Inconel 600 region and a $500 \mathrm{~N}$ load was used in the SiC region. The resulting indentations were measured, and the calculated hardness value was recorded. Multiple indents were taken to get an estimated average within each region. 
Figure 24 shows the shape of the indentation from a Vickers microhardness test in two different views, side and top view. The following equation is the formula for the hardness value [18]:

$$
H V=1.854
$$

Where: $d_{1}=d_{2}=d$
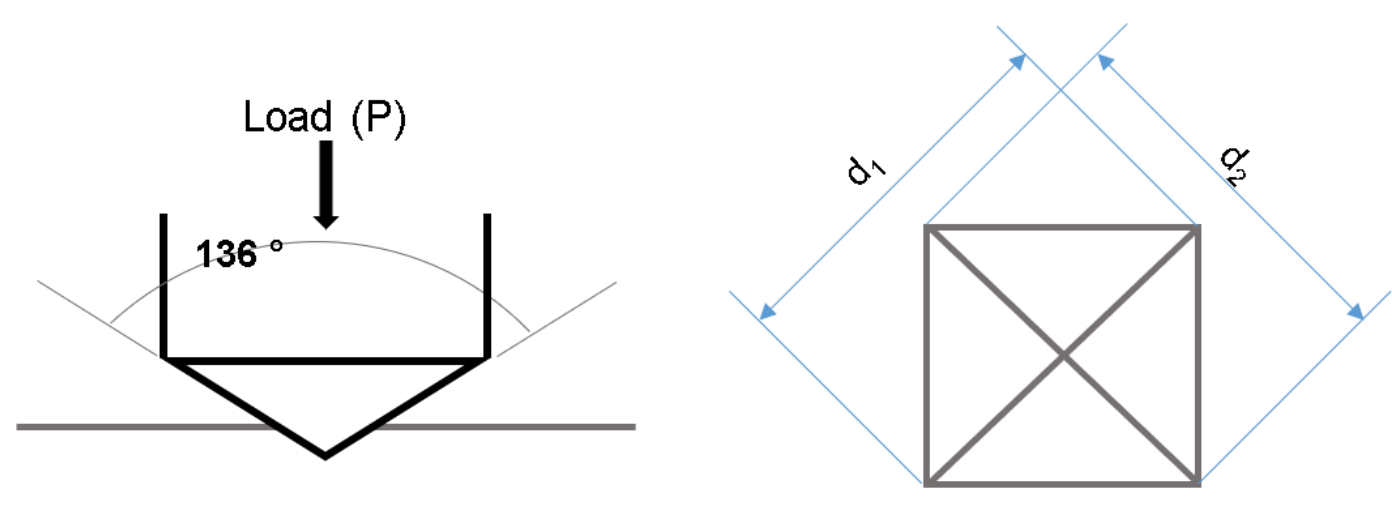

(b)

Figure 24 Schematic representation of a Vickers microhardness indent. Image (a) side view of how diamond pyramid indenter indents the surface of a sample and image (b) top view of indent mark on surface after indenting. Images adapted from Callister [18].

\section{CHAPTER FOUR: RESULTS}

\section{1: Characterization of Starting Materials}

Before characterization, materials were prepared to minimize surface contamination and ensure the results represented fully the materials used in this study before the diffusion bonding process. Pellets of both $\mathrm{SiC}$ and Inconel 600, were polished by hand from 800 to 1200 grit $\mathrm{SiC}$ paper and then by 9 and $3 \mu \mathrm{m}$ diamond paste waterbased suspension. Once polished, pellets were rinsed in isopropanol (IPA), DI water, and ethanol sequentially. 


\subsection{1: Inconel 600}

The as-received Inconel 600 was characterized by EDS point scans $\pm 2 \mathrm{wt} \%$ of $\mathrm{Cr}$, Fe, and Ni content. Table 8 shows the concentration of 10 different point scans taken in different regions of the Inconel 600 pellet. The concentration profile obtained by EDS was consistent with the information provided by the manufacturer $\pm 2 \mathrm{wt} \%$ of $\mathrm{Cr}, \mathrm{Fe}$, and Ni content.

\section{Table 8 Inconel 600 concentration in wt \% table from 10 EDS point scans on} as-received pellet.

\begin{tabular}{|c|c|c|c|}
\hline Concentration in wt\% & $\mathrm{Cr}$ & $\mathrm{Fe}$ & $\mathrm{Ni}$ \\
\hline Average & 16 & 9 & 75 \\
\hline Manufacturer's Data [29] & 15.77 & 8.96 & 73.97 \\
\hline
\end{tabular}

In addition, XRD was performed on the as-received Inconel 600 pellet. The

resultant pattern is shown in Figure 25. As expected, the Inconel 600 diffraction pattern indicated that nickel is the main phase present, since nickel consists of $74 \mathrm{wt} \%$ of the full composition. However, the peaks do not perfectly align to the pdf Ni pattern positions since the presence of $\mathrm{Cr}$ and $\mathrm{Fe}$ within the solid solution structure may alter the shift because the ratio of the $\mathrm{Ni}$ is not purely $100 \%$. 


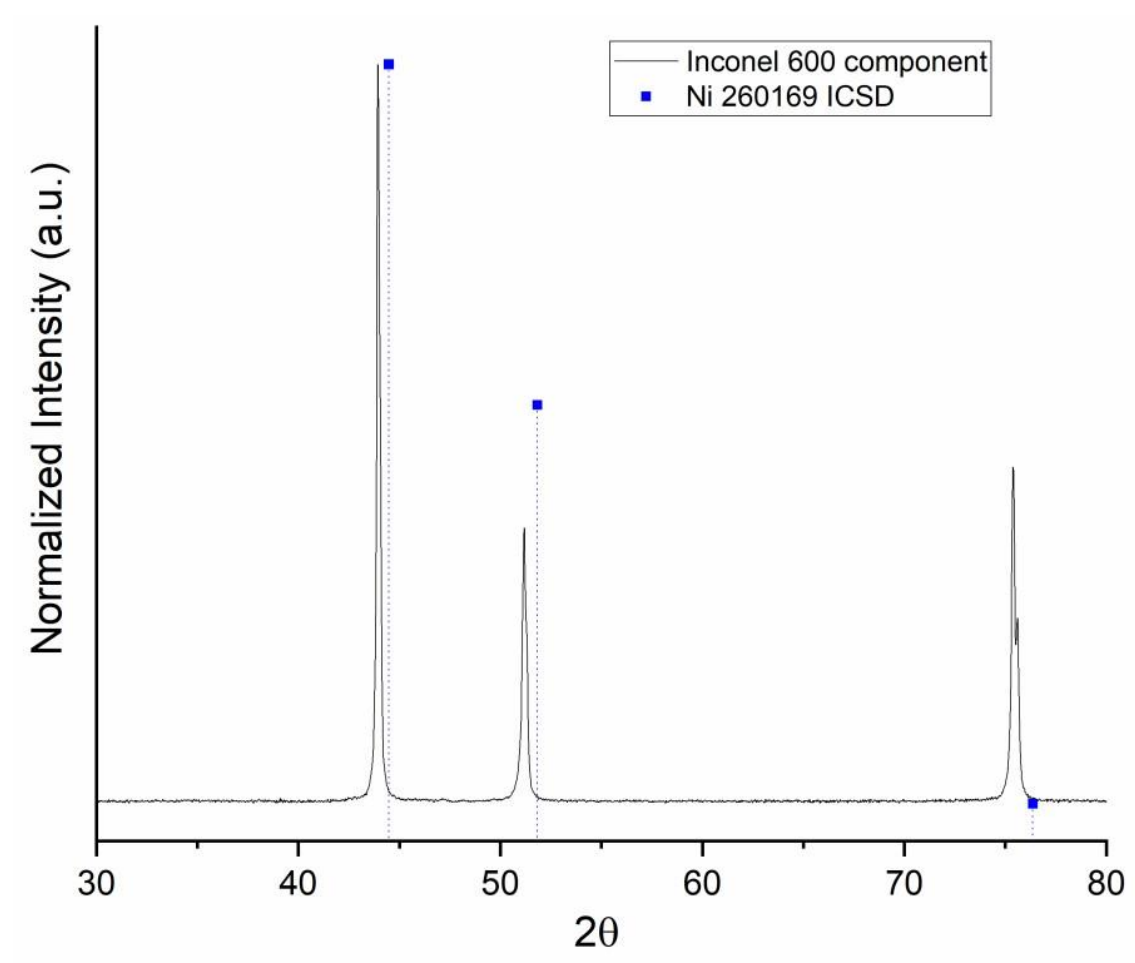

Figure 25 XRD data of Inconel 600 as-received pellet. This pattern was used when characterizing the fracture surfaces of the Inconel 600 side. The shift observed is due to the slightly different lattice parameter of the Inconel 600 structure in comparison to the pure FCC structure due to the concentration of some Fe and $\mathrm{Cr}$ atoms within the structure. The presence of Fe and $\mathrm{Cr}$ within the Ni FCC structure do not change the crystal structure of the alloy, since Inconel 600 is a solid solution and the crystal structure remains unchanged from the structure of the base element, the lattice is affected by the difference in atomic radius.

Since the ICSD database did not have the Inconel 600 pattern file, results were compared to work done by Ravindra Kumar, et al. [39] and by Liu Wei, at el. [40]. XRD Results obtained by the respective authors were similar to the XRD pattern of the Inconel 600 alloy shown in Figure 25. These results are shown in Figure 26, where plot (a) shows the shift that the Inconel 600 pattern experienced with temperature increases. On the other hand, plot (b) shows only the XRD pattern of the Inconel 600 pattern and the gamma phase pattern. 


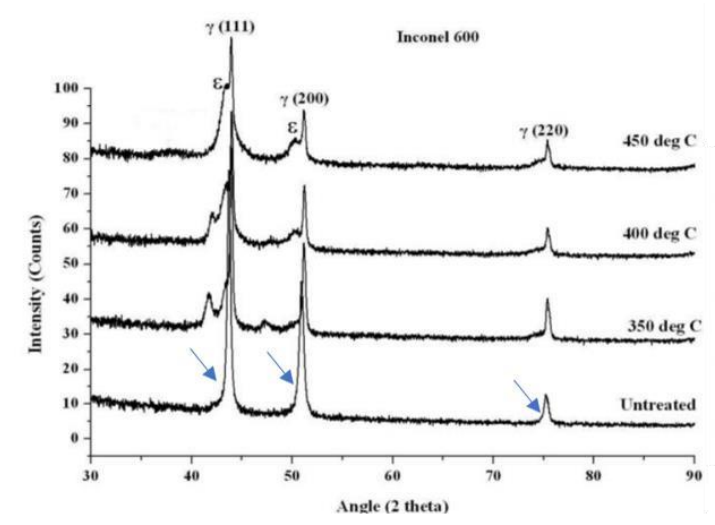

(a)

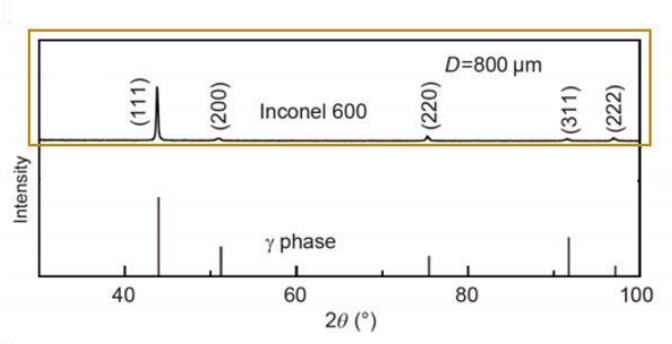

(b)

Figure 26 XRD patterns of Inconel 600 showing the three expected peaks at $44^{\circ}$, $51^{\circ}$, and $75^{\circ}$ from (a) study done by Y.C.S. Ravindra Kumar, et al. and (b) study done by W. Liu, et al. These Images were modified from $[39,40]$ and combined here.

\subsection{2: Reaction Bonded Silicon Carbide (SiC)}

In reaction bonded silicon carbide, free silicon is expected within the $\mathrm{SiC}$ matrix.

The amount of free silicon was characterized using SEM and EDS. Figure 27 shows typical SEM images of the SiSiC material used in the bonding experiments.
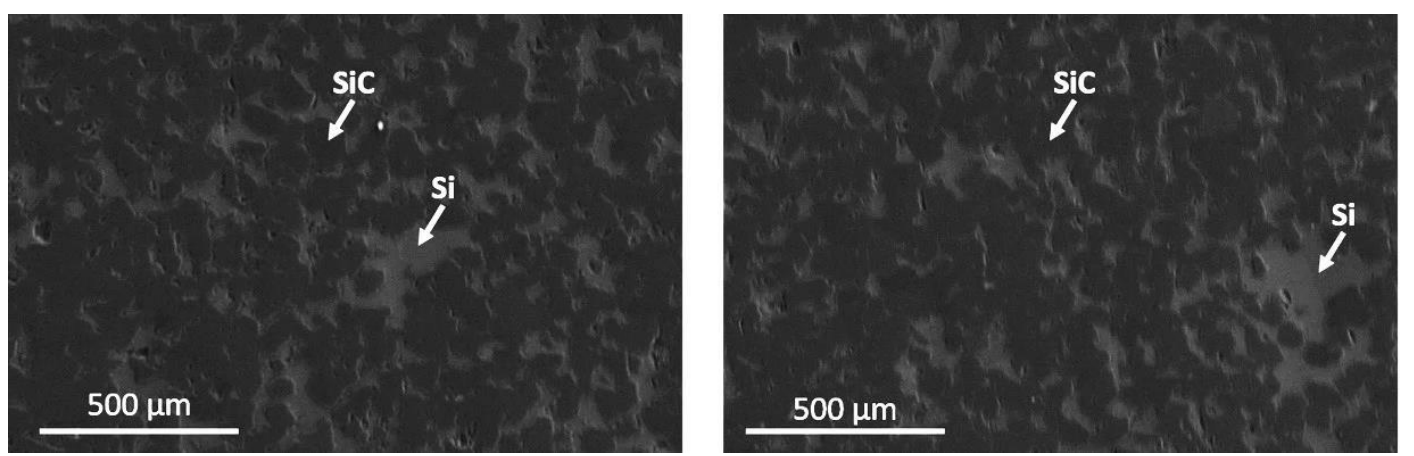

Figure 27 Two SEM images of a cross section of a $\mathrm{SiC}$ rod received from

Coorstek showing free $\mathrm{Si}$ in the $\mathrm{SiC}$ material. These images were used to calculate the amount of free $\mathrm{Si}$ present within the $\mathrm{SiC}$ component.

Several EDS point scans were taken to validate the composition of each of the two different phases shown in Figure 27. The light phase corresponds to the free Si phase, and the darker region corresponds to the $\mathrm{SiC}$ phase. EDS validated the average composition of Si to be $100 \%$ in the light phase and the dark phase (SiC) to be $47 \pm 1 \%$ Si and $53 \pm 1$ 
$\mathrm{C} \%$. The $\%$ error is representative of the standard deviation calculated from all the collected values.

An image processing software (e.g. Fiji/ImageJ) was used to extract the proportion of the two phases in the $\mathrm{SiC}$ component as shown in Figure 28 (the elemental $\mathrm{Si}$ phase present as compared to the $\mathrm{SiC}$ ). The elemental $\mathrm{Si}$ was calculated to be 17.5 $\mathrm{wt} \%$ and $\mathrm{SiC}$ was $82.5 \mathrm{wt} \%$ of the as-received sample by using equations (4.1) and (4.2) below.

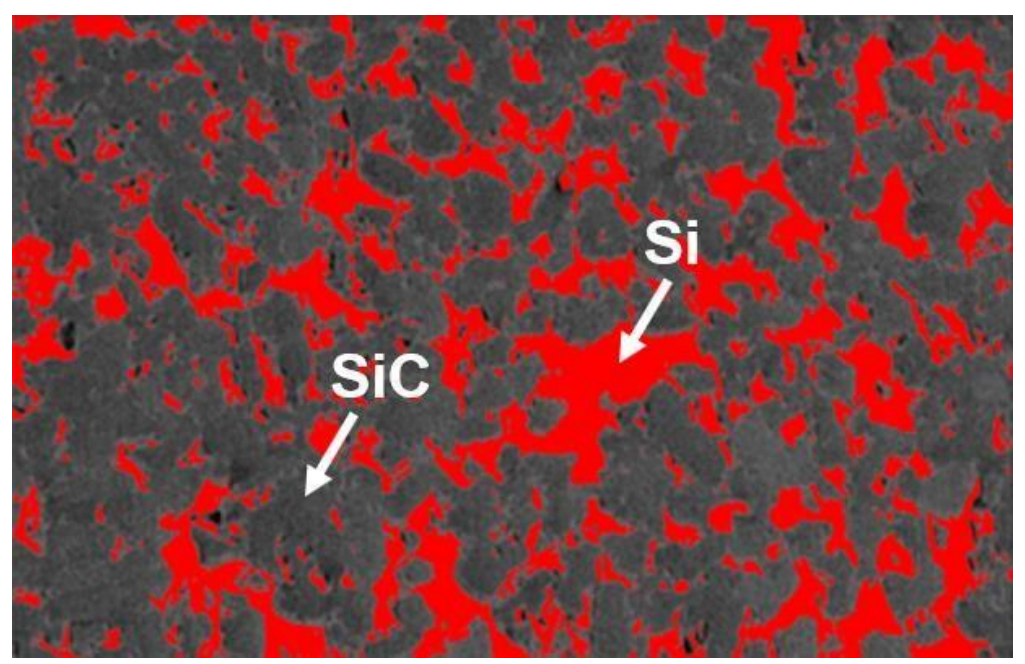

Figure 28 Typical Fiji processing software image showing the threshold colored image. The two different colors within the image correspond to two different phases and allow calculation of the \% area of the red phase, which is the free $\mathrm{Si}$ phase.

$$
\begin{aligned}
& \text { Wt } \% \mathrm{X} \text { in } \mathrm{Y}=\frac{\rho_{\mathrm{X}} * \% \text { Area of } \mathrm{X}}{\rho_{\mathrm{X}} * \% \text { Area of } \mathrm{X}+\rho_{\mathrm{Y}} * \% \text { Area of } \mathrm{Y}} \\
& \text { Wt } \% \text { Si in sample }=\frac{\rho_{\mathrm{Si}} \% \mathrm{Area} \text { of Si }}{\rho_{\mathrm{Si}} \% \text { Area of } \mathrm{Si}+\rho_{\mathrm{SiC}} \% \text { Area of SiC }} * 100
\end{aligned}
$$


In addition, $\mathrm{XRD}$ was performed on the as-received $\mathrm{SiC}$ pellet, and the resultant pattern is shown in Figure 29, showing the multiple phase structure as well as indicating that the crystal structure of the $\mathrm{SiC}$ matrix is mixed between the $6 \mathrm{H}$ and $4 \mathrm{H} \mathrm{SiC}$ crystal structure.

The pattern was compared to the ICSD pattern for $\mathrm{Si}, 4 \mathrm{H} \mathrm{SiC}$ and $6 \mathrm{H} \mathrm{SiC}$.

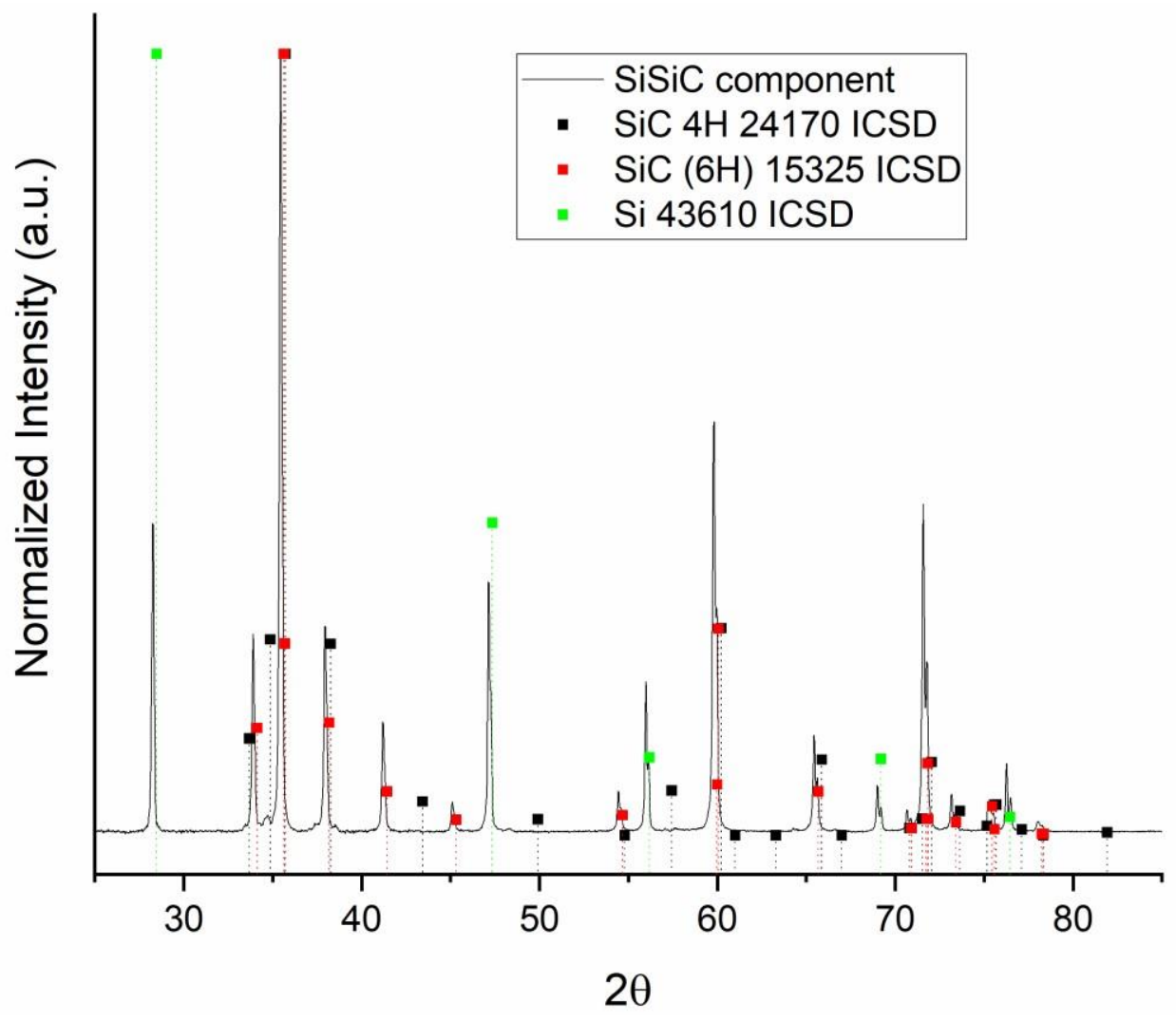

Figure 29 XRD data of the $\mathrm{SiC}$ as-received pellet. The red squares described the $\mathrm{SiC}(6 \mathrm{H})$ crystal structure peaks positions, the black squares represent the $\mathrm{Si}(\mathbf{4 H})$ crystal structure peaks positions and the blue circles represent the $\mathrm{Si}$ peaks positions from the expected free $\mathrm{Si}$ present within the $\mathrm{Si}-\mathrm{SiC}$ matrix.

\subsection{3: Interlayer Materials}

The Ag foil is specified as 99.9 wt\% silver with a thickness of 0.01 inches or 0.25 $\mathrm{mm}$ from the supplier, Rio Grande [34]. Ten EDS point scans were taken to validate the concentration profile of the purchased silver interlayer. EDS validated that the average composition was $100 \% \mathrm{Ag}$, given the limits of detection. XRD was performed and the resultant pattern of the as-received interlayer material is shown in Figure 30. This pattern 
also validates the purity of the Ag foil purchased, since the pattern only has peaks that can be associated with pure Ag. The XRD pattern was also used for comparison when examining the fracture surfaces from the samples following joining and tensile testing.

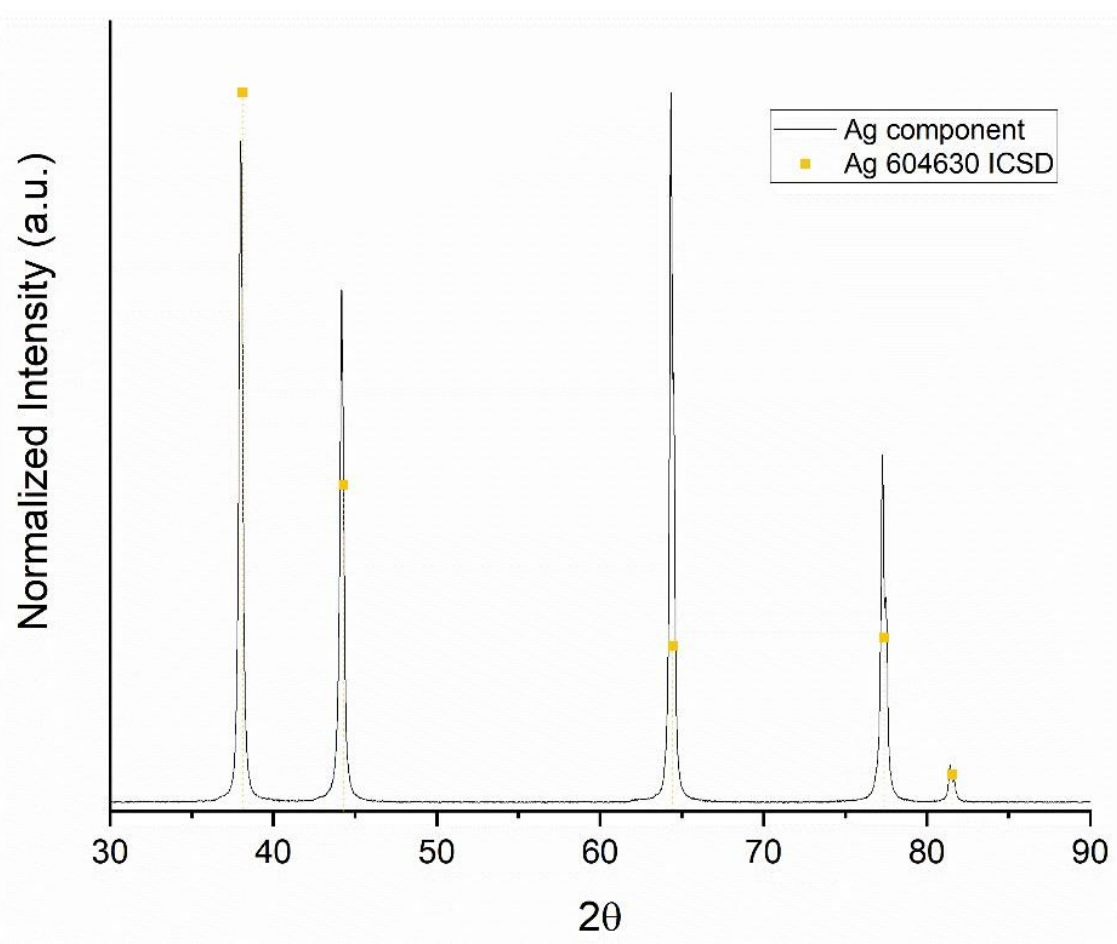

Figure $30 \quad$ XRD data of as-received Ag interlayer.

The as-received $75 \% \mathrm{Ag}-25 \% \mathrm{Pd}$ alloy interlayer from ESPI Metals was also characterized via EDS point scans to get an initial quantitative description of the composition of each interlayer material and to validate the vendor's data sheets. Several scans were taken to characterize the $\mathrm{Ag}_{75} \mathrm{Pd}_{25}$ interlayer and the values are given in Table

9. In addition, the XRD spectrum of the as-received interlayer material is shown in Figure 31 showing the peaks corresponding to the solid solution alloy crystal structure, as well as some extra phases present within the material.

Table 9 Silver-Palladium $25 \%$ alloy concentration table from EDS point scans on an as-received $75 \% \mathrm{Ag}-25 \% \mathrm{Pd}$ pellet. 


\begin{tabular}{|l|l|l|}
\hline Point & $\mathrm{Pd}$ & $\mathrm{Ag}$ \\
\hline Average & 22 & 78 \\
\hline Manufactured & $25 \pm 2 \%$ & $75 \pm 2 \%$ \\
\hline
\end{tabular}

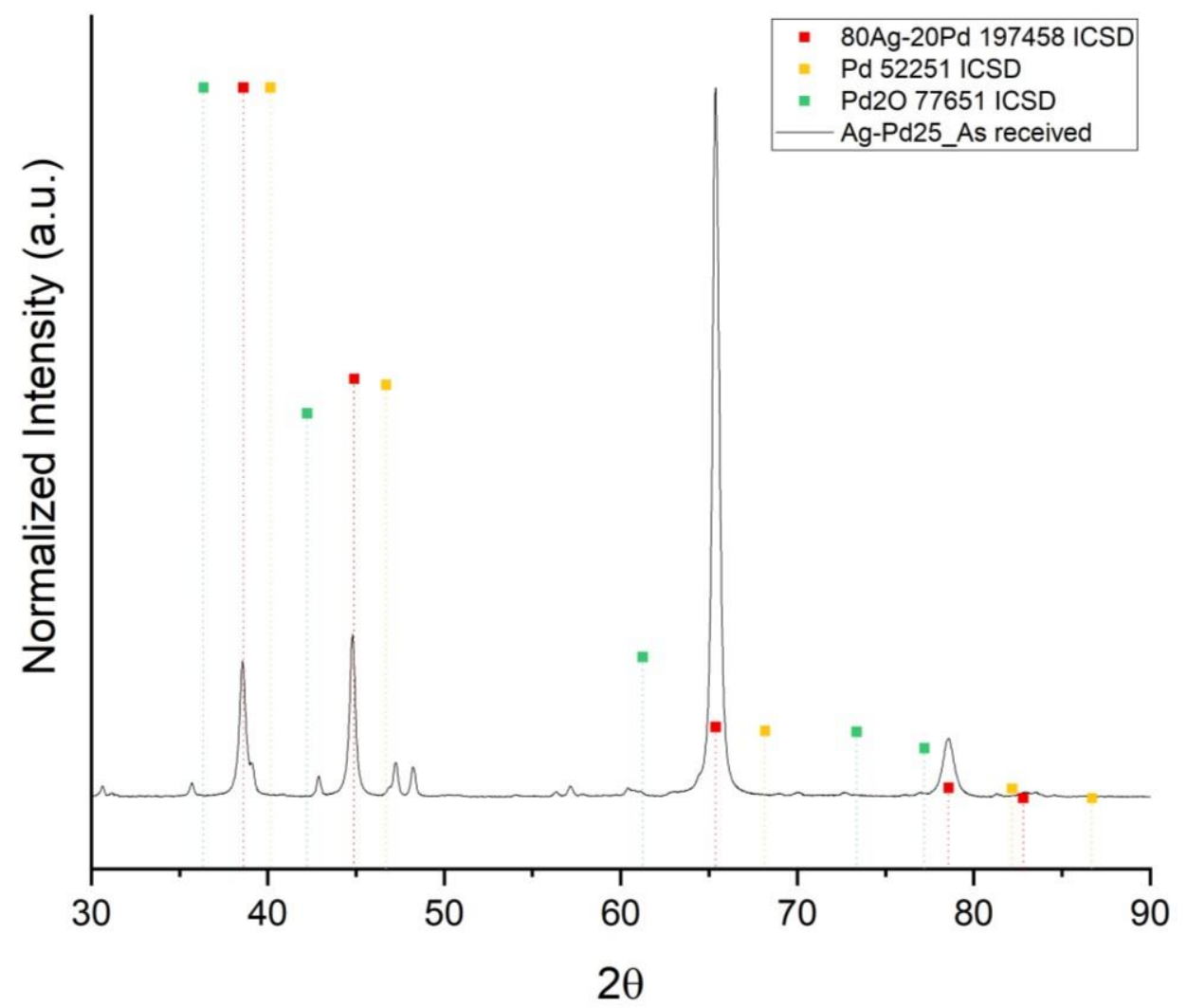

Figure 31 XRD data of the Ag-Pd 25\% alloy as-received interlayer. Some minor peaks are unknown, however the major peaks are indexed and represent a 80Ag20Pd alloy phase, as well as some extra Pd phase.

\section{2: Interfacial Microstructure Characterization}

This section introduces the results from the diffusion bonded Inconel 600 to SiC transitions fabricated at different process conditions (Table 7) such as, temperatures of $900{ }^{\circ} \mathrm{C}$ or $930^{\circ} \mathrm{C}$, pressures of $1 \mathrm{MPa}$ or $10 \mathrm{MPa}$, and times of $30 \mathrm{~min}$ or $180 \mathrm{~min}$ and using two different interlayer materials, such as $\mathrm{Ag}$ and $\mathrm{Ag}-\mathrm{Pd} 25 \%$ alloy.

Figure 32 shows the cross-section SEM images of four joints (A-D) formed at different process parameters combinations (Table 7) and using the Ag interlayer material. 
In Figure 32, images A and B show the side by side comparison of the two joints fabricated under different pressure conditions (same temperature and time) in order to understand the effect of pressure on the microstructure. The increase in pressure affects the mass transfer of the $\mathrm{Ag}$ interlayer into the $\mathrm{SiC}$ component which is labeled as reaction region (2). In addition, increased pressure affects the formation of cracks along the interface between the Ag interlayer and the Inconel 600. This cracked region is observed to be different than the bulk Inconel 600 and it is labeled as reaction region (1). Finally, a major observation between image A and B is that the Ag interlayer appears to be fully consumed during the bonding process. In Figure 32, images B and D are as top to bottom comparison of the two joints fabricated under different temperature conditions (same pressure and same time). The increased temperature results in the formation of a new reaction region labeled as (2) in image D at the interface. Also, image D shows less cracking than image B. Lastly, Figure 32 images C and D show the side by side comparison of joints fabricated for different bonding times (same temperature and same pressure) and how bonding time affected the microstructure of the joints. The increased time at the temperature of $930^{\circ} \mathrm{C}$ results in wider reaction layers. Also, image $\mathrm{C}$ shows cracking throughout reaction region (4) that previous images do not show, as well as, a new reaction region labeled as (2). The white boxes shown in images B and C correspond to higher magnification SEM images for further observations shown in Figure 34 and Figure 35 . 

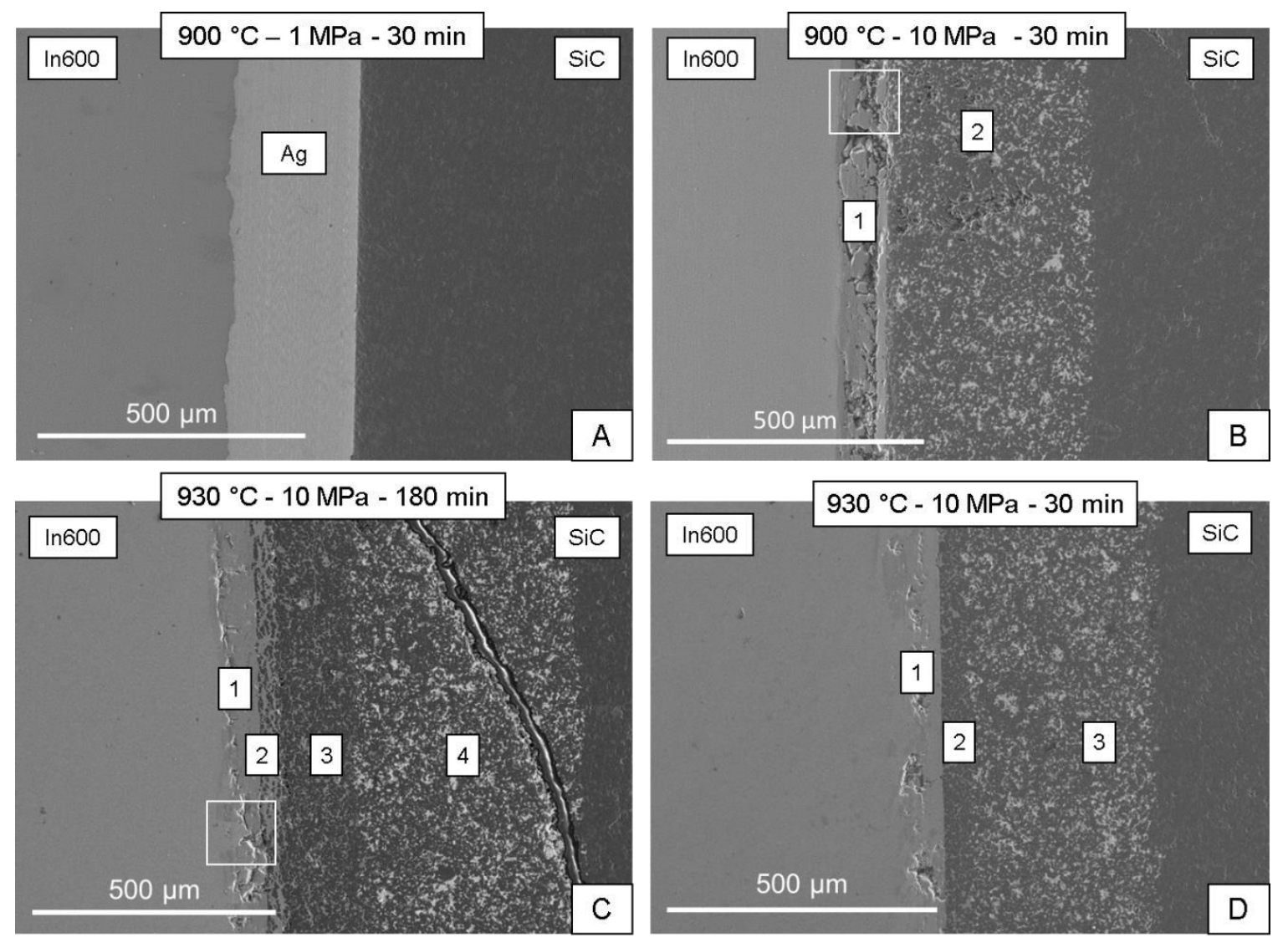

Figure 32 Secondary electron SEM cross-section images showing the morphology at the interface of each bonded Inconel $600 / \mathrm{Ag} / \mathrm{SiC}$ joint: (A) $900{ }^{\circ} \mathrm{C}$ under $1 \mathrm{MPa}$ for 30 minutes, (B) $900{ }^{\circ} \mathrm{C}$ under $10 \mathrm{MPa}$ for 30 minutes, (C) $930{ }^{\circ} \mathrm{C}$ under $10 \mathrm{MPa}$ for 30 minutes, (D) $930{ }^{\circ} \mathrm{C}$ under $10 \mathrm{MPa}$ for 180 minutes.

Figure 33 shows four cross-section SEM images of the joints formed using 900 ${ }^{\circ} \mathrm{C}, 1 \mathrm{MPa}$, and $30 \mathrm{~min} .900{ }^{\circ} \mathrm{C}$ was the lowest temperature used for bonding in these experiments. Although adhesion between the components was achieved, very limited reactions are seen in the samples bonded at these conditions. For example, Figure 33 image (1) shows no diffusion or interfacial chemical reactions across the interface in either direction. Some plastic deformation was observed at the interlayer-metal interface indexed with black arrows. Images (a) and (b) are of the joint in (1) at slightly greater magnification. These images indicate that limited or no mass diffusion or chemical reactions occur at the interfaces under these conditions. On the other hand, image (2), 
which corresponds to a different joint fabricated using the same process parameter combination, shows some diffusion across the interlayer/SiC interface. Two regions are labeled, one as $\mathrm{Ag}+\mathrm{Si}$ and the second as reaction layer $\mathrm{C}$.

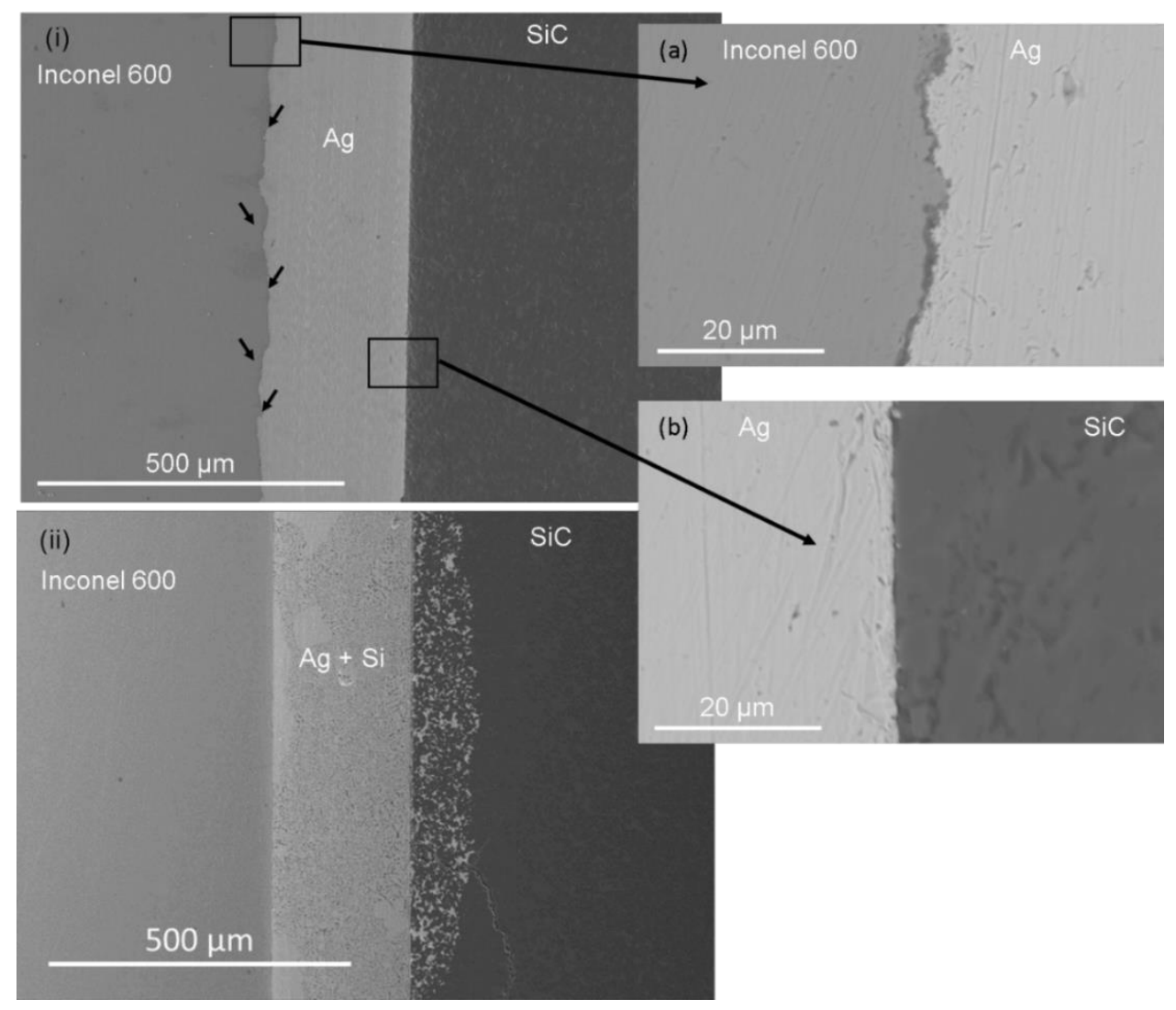

Figure 33 Two secondary electron SEM cross-section images showing joints formed at $900{ }^{\circ} \mathrm{C}$ under $1 \mathrm{MPa}(50 \mathrm{~N})$ for 30 minutes with $\mathrm{Ag}$ as the interlayer image (i) showing no diffusion but plastic deformation of the interlayer material and image (ii) showing a small diffusion as well as a small crack. SEM cross-section images, (a) shows the metal-metal interface and (b) shows the metal-ceramic interface of image (1).

Figure 34 is a Backscattered Electron (BSE) cross-section SEM image of the joint shown in Figure 32 (B), bonded at $900{ }^{\circ} \mathrm{C}, 10 \mathrm{MPa}$, and 30 minutes. In reaction region (1) cracking is seen which relates to the presence of multiple reactions at the Inconel 600/SiC interface. Several point scans were taken at each reaction region ([i], [ii], [iii], [iv], and [v]) within, to quantitively investigate the composition of each area. 
Table 10 shows the composition ratios obtained by EDS point scans in each reaction region.

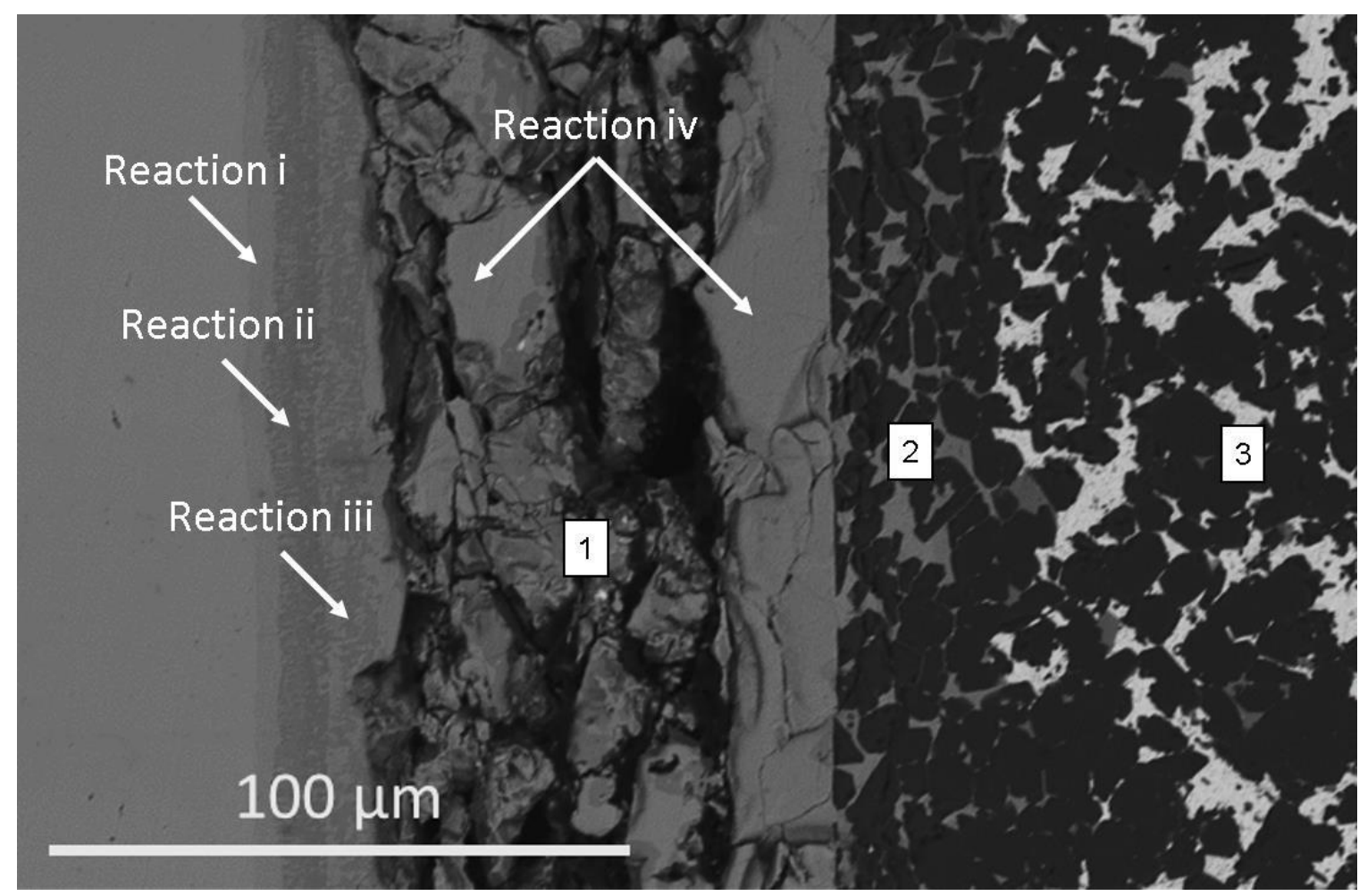

Figure 34 Higher magnification of Figure 32 (B) showing the BSE SEM image where different reaction layers are observed. These regions observed within the reaction region show cracking within them. Compositions at each is collected and given in

Table 10.

In addition, the starting of a reaction region labeled as (2) is observed at this magnification that was not observed at the magnification used in Figure 32 (B). This SEM image also shows some reaction region labeled as (3) that correspond to reaction region labeled as (2) in Figure 32 (B).

Table 10 Elemental concentration from EDS point scans from Figure 34 for the joint formed at $900{ }^{\circ} \mathrm{C}$ under $10 \mathrm{MPa}$ for 30 minutes with the Ag-interlayer.

\begin{tabular}{|c|c|c|c|c|}
\hline Reaction & $\mathrm{Si}$ & $\mathrm{Cr}$ & $\mathrm{Fe}$ & $\mathrm{Ni}$ \\
\hline $\mathrm{i}$ & 21 & 18 & 10 & 51 \\
\hline $\mathrm{ii}$ & 24 & 61 & 4 & 11 \\
\hline
\end{tabular}




\begin{tabular}{|c|c|c|c|c|}
\hline iii & 40 & 27 & 10 & 23 \\
\hline iv & 48 & 6 & 16 & 30 \\
\hline V & 38 & 5 & 3 & 59 \\
\hline
\end{tabular}

Figure 35 shows the cross-section SEM image of the joint formed at $930{ }^{\circ} \mathrm{C}, 10$

MPa and 180 minutes shown in Figure 32 (C). To understand what the composition profiles of the different observed reactions was like, the EDS chemical maps were taken. These EDS map scans are also shown and from them the following observations were made:

- Concentration of Si is confirmed by the EDS map scan within the Inconel 600 component.

- Regions with high $\mathrm{Cr}$ concentration and low Ni concentration are shown and mapped.

- Similar to Figure 34, multiple different reaction layers are observed at the interface.

- EDS map scans show high concentration spots of Ag by the intensity of the red color in comparison to the background.

In addition to the EDS map scans shown in Figure 35, quantitative EDS point scans were taken at each region of interest to support in conjunction the observations presented above. These average values are given in Table 11. 

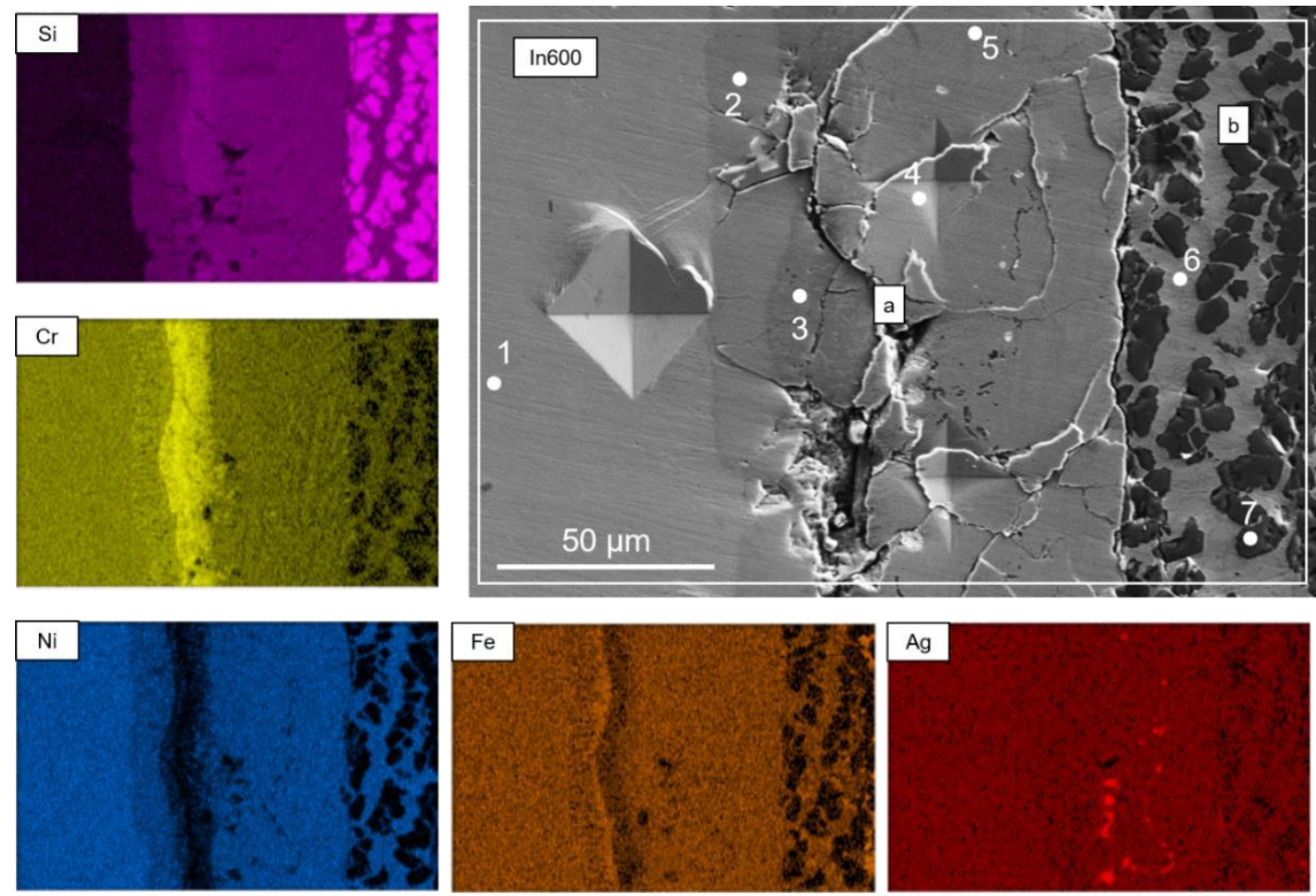

Figure 35 EDS map scans showing chemical concentration gradients of the elements of joint shown in Figure $32(\mathrm{C})$.

Table 11 EDS point scans from Figure 35 from reaction layers $A$ and $B$ representing the joint formed at $930{ }^{\circ} \mathrm{C}$ under $10 \mathrm{MPa}$ for 180 minutes with an Aginterlayer.

\begin{tabular}{|c|c|c|c|c|c|c|}
\hline Region & $\mathrm{C}$ & $\mathrm{Si}$ & $\mathrm{Cr}$ & $\mathrm{Fe}$ & $\mathrm{Ni}$ & $\mathrm{Ag}$ \\
\hline 1 & - & - & 18 & 9 & 73 & - \\
\hline 2 & - & 20 & 22 & 10 & 45 & - \\
\hline 3 & - & 24 & 71 & 3 & 1 & - \\
\hline 4 & - & 13 & 12 & 4 & 16 & 40 \\
\hline 5 and 6 & - & 33 & 6 & 7 & 55 & - \\
\hline 7 & 57 & 42 & - & - & 1 & - \\
\hline
\end{tabular}

In summary, the main observations from the characterization of the joints

fabricated with the Ag interlayer material are that:

- As temperature, time, and pressure are increased, more reactions are seen, the reaction layers become thicker and more diffusion is observed.

- Multiple reaction phases are observed at the interface as the process parameters increased. 
- Cracking is observed along the interface where reactions occur.

- A separate Ag layer is no longer observed except in joints formed at 900 ${ }^{\circ} \mathrm{C}, 1 \mathrm{MPa}$ and 30 minutes.

Figure 36 shows a side by side view of four cross-section SEM images of joints formed with a $75 \% \mathrm{Ag}-25 \% \mathrm{Pd}$ interlayer and the four different combinations of process parameters given in Table 7. Images A and B show how a pressure increase affects the interlayer formation. In image B, the $75 \% \mathrm{Ag}-25 \% \mathrm{Pd}$ interlayer material is no longer visible at the interface. Cracks or voids can be observed at the interface where a labeled ( $\left.1^{\prime}\right)$ reaction region exists. A reaction layer labeled as (3' and 2') is observed in both images A and B respectively, and the thickness is greater in image B when compared to image A. Images B and D (increasing temperature) show the formation of a much larger reaction layer labeled as (2' and 3'), again respectively. In addition, in image D two reaction layers labeled as (1') and (2') are observed, as well as minor cracking along these reaction layers. Image $\mathrm{C}$ and $\mathrm{D}$ show the effect of bonding time on the microstructure of the joints. With increasing bonding time, the reaction layer growth is clearly observed. Also, major cracking along the metal/ceramic interface can be observed in image $\mathrm{C}$ when compared to the cracking observed in any of the other three images. 

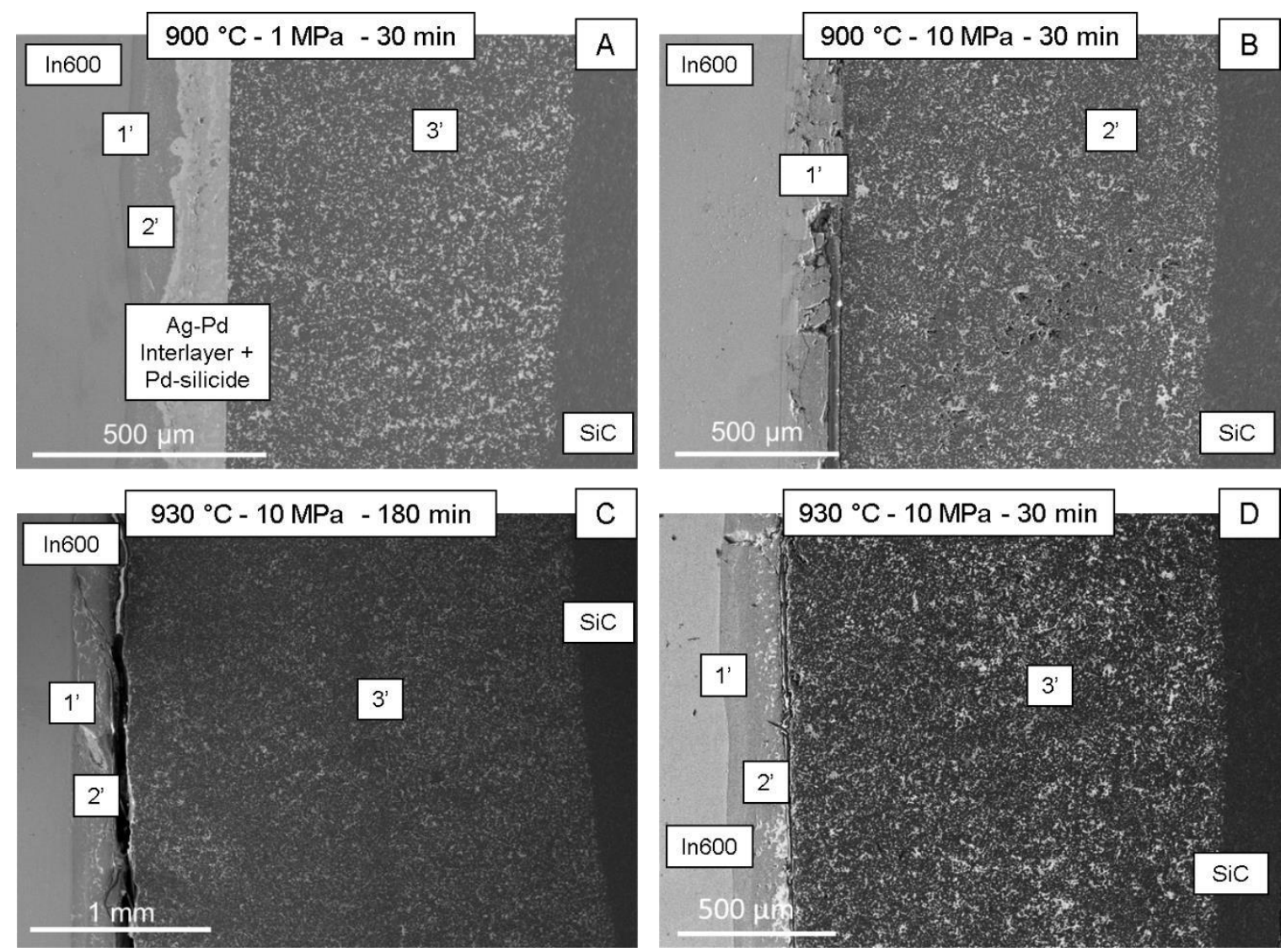

Figure 36 Secondary electron SEM cross-section images showing the morphology at the interface of each bonded Inconel 600/AgPd/SiC joint fabricated at different combinations of process parameters, shown in Table 7 . The magnifications used for each image is different to show all the different distinct regions within each of the joints.

Figure 37 shows the cross-section SEM image of the joint formed at $900{ }^{\circ} \mathrm{C}$ under a load of $1 \mathrm{MPa}$ for 30 minutes (Figure $36 \mathrm{~A}$ ) with EDS map scans and an EDS line scan. The EDS map scans revealed regions with high $\mathrm{Cr}$ concentration and low $\mathrm{Ni}$ concentration and vice versa. Similarly, the EDS map scans show diffusion of Si into the Inconel 600. The EDS line scan indicates that reactions are occurring due to mass transfer in either direction. By comparing the SEM image with the EDS line scan, several trends are seen. In the center region, the $\mathrm{Ag}$ and $\mathrm{Pd}$ are no longer evenly distributed. When the Ag concentration is high, the Pd concentration is low. This suggests the formation of a 
Ag rich phase, while $\mathrm{Pd}$ concentration and $\mathrm{Si}$ concentration are both high in certain areas, suggesting formation of a palladium silicide and the depletion of Ag.

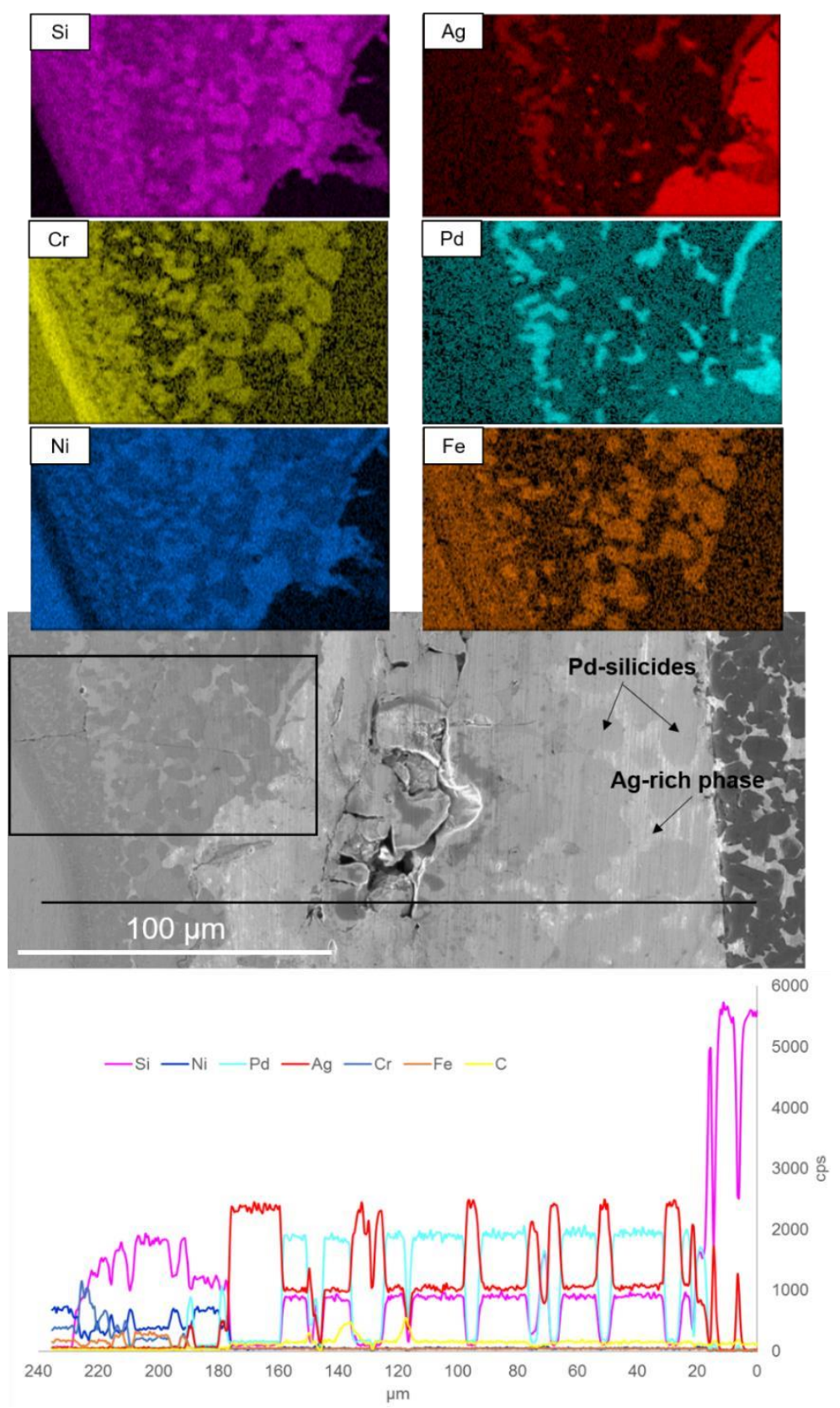

Figure 37 Secondary electron SEM cross-section image, EDS map scans and EDS line scan of the joint formed at $900{ }^{\circ} \mathrm{C}$ under $1 \mathrm{MPa}$ for 30 minutes with 75\% Ag-25\% Pd interlayer shown in Figure 36 (A). This SEM images corresponds to the white box shown in Figure 36(A) at a higher magnification to take further observations. 
Table 12 shows the collected concentrations within different regions of a joint fabricated at $900{ }^{\circ} \mathrm{C}$ under $1 \mathrm{MPa}$ for 30 minutes with a $75 \% \mathrm{Ag}$ - 25\% Pd interlayer from Figure 38, which shows a different region along the interface of this joint. Multiple phases are observed at this magnification.

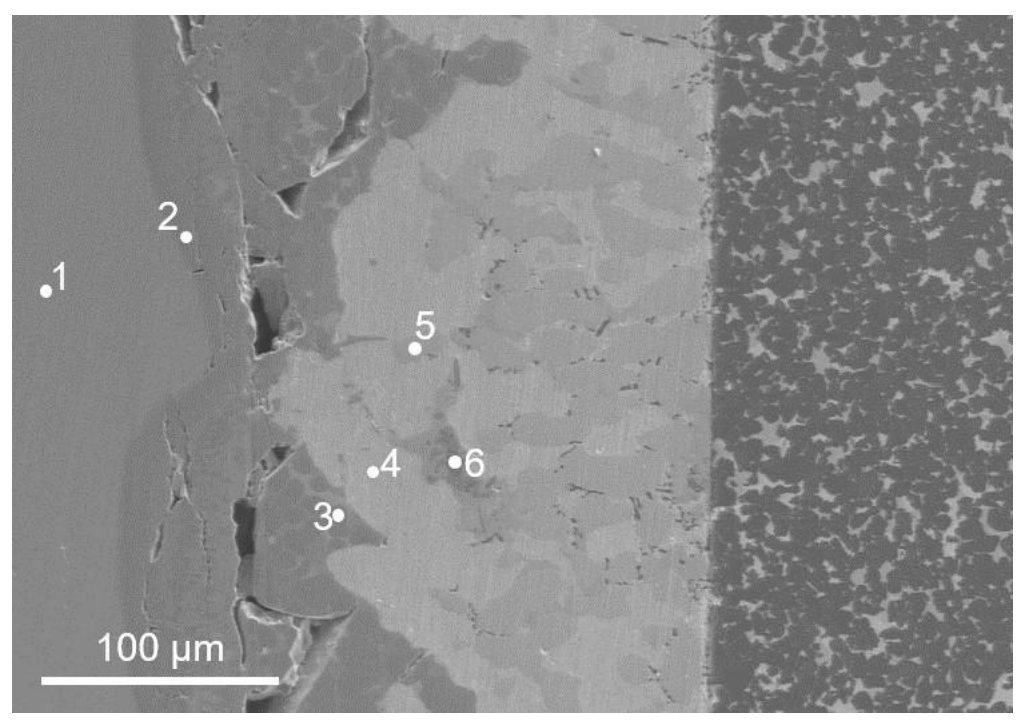

Figure 38 Secondary electron cross-section SEM image of joint formed at 900 ${ }^{\circ} \mathrm{C}$ under $1 \mathrm{MPa}$ for 30 minutes with $75 \% \mathrm{Ag}-25 \% \mathrm{Pd}$ interlayer showing EDS point scans collected representing the different regions within the reaction layer that are observed within the Inconel 600 side.

Table 12 EDS point scans from the joint formed at $900{ }^{\circ} \mathrm{C}$ under $1 \mathrm{MPa}$ for 30 minutes with a $75 \% \mathrm{Ag}-25 \% \mathrm{Pd}$ interlayer.

\begin{tabular}{|c|c|c|c|c|c|c|}
\hline Point & $\mathrm{Si}$ & $\mathrm{Cr}$ & $\mathrm{Fe}$ & $\mathrm{Ni}$ & $\mathrm{Pd}$ & $\mathrm{Ag}$ \\
\hline 1 & - & 18 & 9 & 73 & - & - \\
\hline 2 & 23 & 49 & 6 & 21 & - & - \\
\hline 3 & 48 & 9 & 11 & 31 & - & - \\
\hline 4 & - & - & - & - & - & 100 \\
\hline 5 & 32 & - & - & 2 & 61 & 6 \\
\hline 6 & 61 & 26 & 1 & 3 & 9 & - \\
\hline
\end{tabular}

Figure 39 shows a higher magnification SEM image of reaction layers a' and b' from the joint formed at $930{ }^{\circ} \mathrm{C}$ under a load of $10 \mathrm{MPa}$ for 180 minutes shown in Figure 
36 (C). The formation of a variety of new phases is observed. EDS map scans revealed regions with high $\mathrm{Cr}$ concentration and low $\mathrm{Ni}$ concentration and vice versa. The scans also confirmed concentration of Si within the Inconel 600. The EDS map scans also show a small non-uniform region with high intensity Fe phase that overlaps with some concentrations of Si and Cr. Minor cracking is observed in areas with high Si concentration. Areas with a high concentration of Ag are also observed. In addition to the EDS map scans shown in Figure 39, EDS point scans were taken to determine atomic concentrations and inform what phases may be forming. The concentrations in each region are given in Table 13.
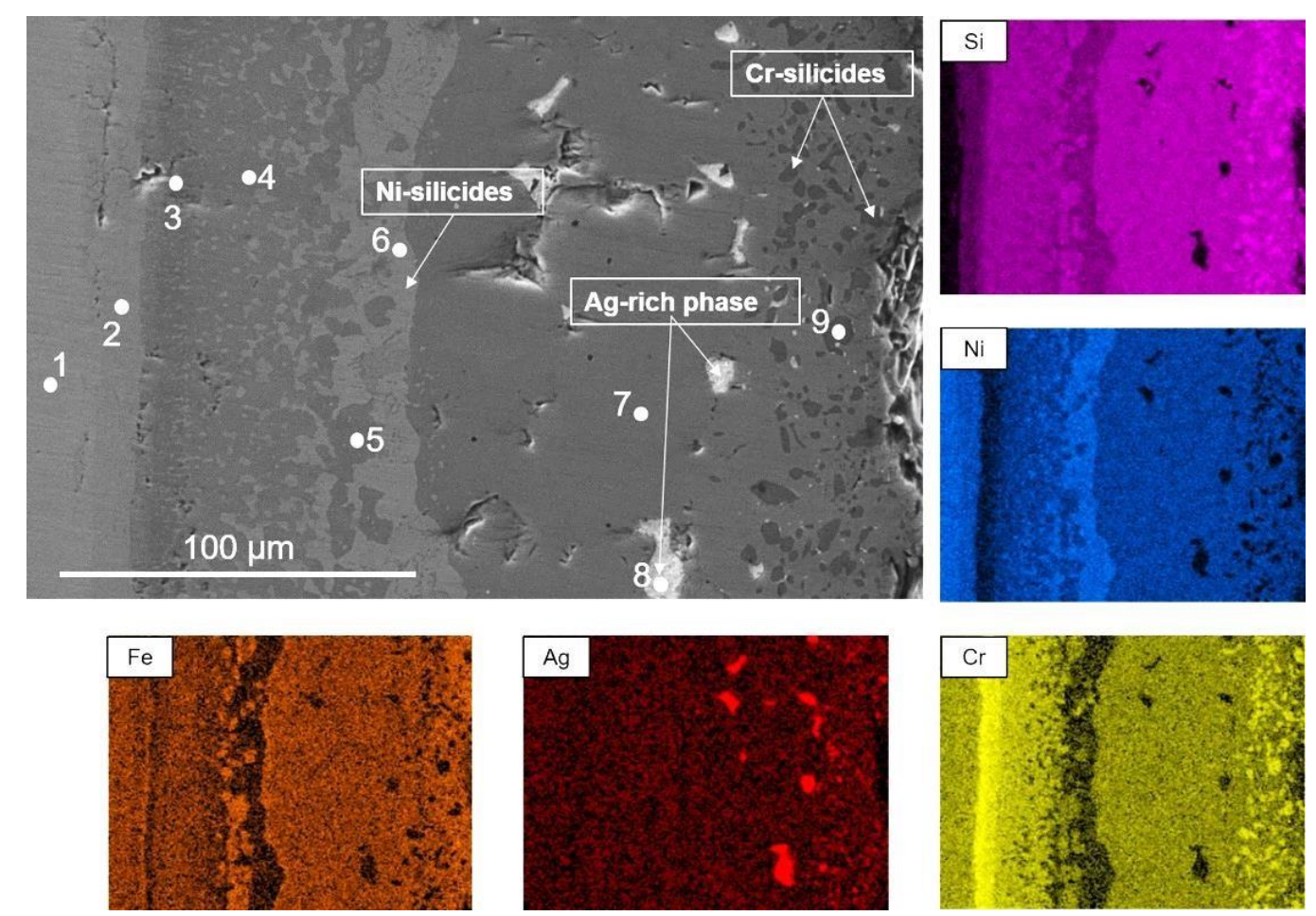

Figure $39 \quad$ Secondary electron cross-section SEM image of joint formed at 930

${ }^{\circ} \mathrm{C}$ under $10 \mathrm{MPa}$ for 180 minutes. EDS map scans showing the different areas with the concentration gradient of elements. This SEM image corresponds to the white box shown in Figure $36(C)$.

Table 13 EDS point scans from Figure 39 from the joint formed at $930{ }^{\circ} \mathrm{C}$ under 10 MPa for 180 minutes with a $75 \% \mathrm{Ag}$ - $25 \%$ Pd interlayer.

\begin{tabular}{|c|c|c|c|c|c|}
\hline Point & $\mathrm{Si}$ & $\mathrm{Cr}$ & $\mathrm{Fe}$ & $\mathrm{Ni}$ & $\mathrm{Ag}$ \\
\hline
\end{tabular}




\begin{tabular}{|c|c|c|c|c|c|}
\hline 1 & - & 18 & 10 & 72 & - \\
\hline 2 & 21 & 17 & 7 & 50 & - \\
\hline 3 & 32 & 44 & 6 & 6 & - \\
\hline 4 & 42 & 24 & 7 & 27 & - \\
\hline 5 & 48 & 10 & 14 & 19 & - \\
\hline 6 & 33 & 2 & 1 & 62 & - \\
\hline 7 & 49 & 10 & 10 & 31 & - \\
\hline 8 & 1 & - & - & 2 & 97 \\
\hline 9 & 65 & 33 & 0 & 1 & - \\
\hline
\end{tabular}

In summary, the main observations from the characterization of the joints

fabricated with a $75 \% \mathrm{Ag}-25 \% \mathrm{Pd}$ interlayer material are that:

- Diffusion and new phases are observed within all the fabricated joints.

- As time, temperature and pressure increase, additional phases, extensive diffusion, and wider reaction layers are observed.

- Cracking becomes more visible along the interface in areas where new phases exist. Cracking is more extensive as time, temperature, and/or pressure increase.

- The interlayer material remains at the interface for the joint fabricated at $900{ }^{\circ} \mathrm{C}, 1 \mathrm{MPa}$, and 30 minutes, although with reactions present and minor cracking. However, once the forming parameters increase, a distinct AgPd layer is no longer present.

\section{3: Comparison between the $\mathrm{Ag}$ and $\mathrm{Ag}_{75} \mathrm{Pd} 25$ interlayer systems}

Two joints formed at $900{ }^{\circ} \mathrm{C}, 1 \mathrm{MPA}$, and 30 min using both the $100 \% \mathrm{Ag}$ and $75 \% \mathrm{Ag}-25 \% \mathrm{Pd} 25 \%$ interlayer and two joints formed at $930^{\circ} \mathrm{C}, 10 \mathrm{MPA}$, and $180 \mathrm{~min}$, also using both interlayers, were used to study the influence of the interlayer on the interfacial microstructure of the joints. Figure 40 shows the resultant microstructure of these four joints. 
Figure 40 (a) shows the joint fabricated at $900^{\circ} \mathrm{C}, 1 \mathrm{MPA}$, and $30 \mathrm{~min}$ with the Ag interlayer, (b) shows the joint fabricated at $930{ }^{\circ} \mathrm{C}, 10 \mathrm{MPA}$, and 180 min using the Ag interlayer, (c) shows the joint fabricated at $900{ }^{\circ} \mathrm{C}, 1 \mathrm{MPA}$, and $30 \mathrm{~min}$ with the $75 \% \mathrm{Ag}-25 \% \mathrm{Pd}$ interlayer (d) joint fabricated at $930{ }^{\circ} \mathrm{C}, 10 \mathrm{MPA}$, and $180 \mathrm{~min}$ using the $75 \% \mathrm{Ag}-25 \% \mathrm{Pd}$ interlayer. All four joints were successfully bonded. However, when compared at increased magnification, the microstructures of the joints formed at $900{ }^{\circ} \mathrm{C}, 1 \mathrm{MPA}$, and $30 \mathrm{~min}$, the $100 \% \mathrm{Ag}$ interlayer did not appear to diffuse and no chemical reactions are seen, while the image of the $75 \% \mathrm{Ag}-25 \% \mathrm{Pd}$ does react. The type of bonding observed on image (a) is achieved by van der Waals forces and charge transfer across the interface $[3,18,19,21,22]$ and is called physical bonding. Because of the applied pressure at $900{ }^{\circ} \mathrm{C}$, localized deformation is seen, and the Ag appears to fill surface imperfections. The type of bonding observed on images $(b-d)$ is achieved by mass transfer and chemical reactions $[3,19,21]$ and is called chemical reaction bonding. The plastic deformation of the interlayer component occurred in both interlayers and is observed in both images in Figure 40 (a) and (d). The interlayer component is no longer visible in images (b) and (d) and it appears that it has completely diffused into the $\mathrm{SiC}$. Cracking is observed through some of the reaction layers in image

(b) and major cracking is observed in image (c). 


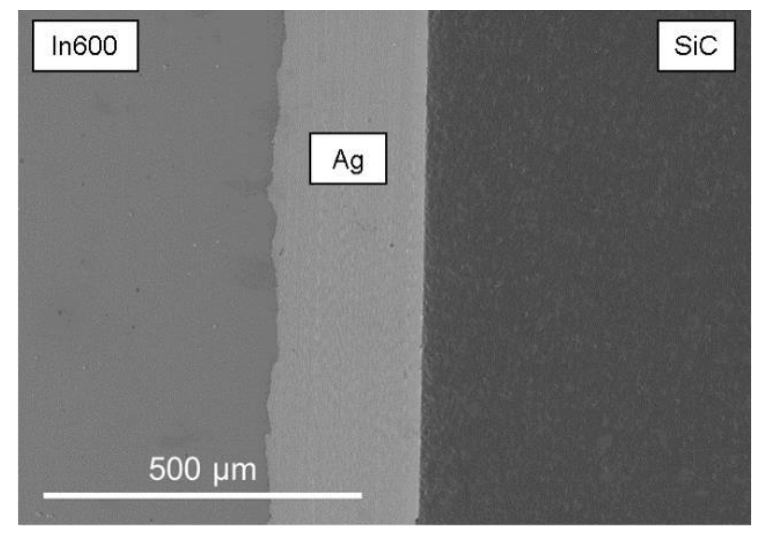

(a)

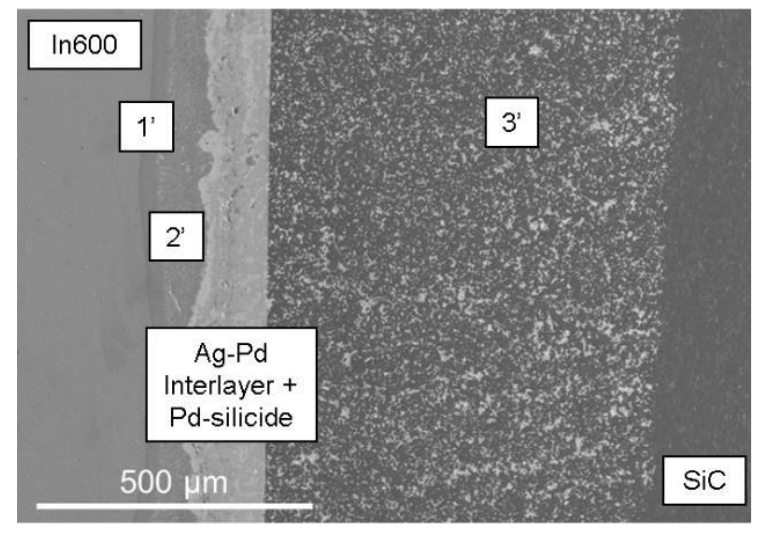

(c)

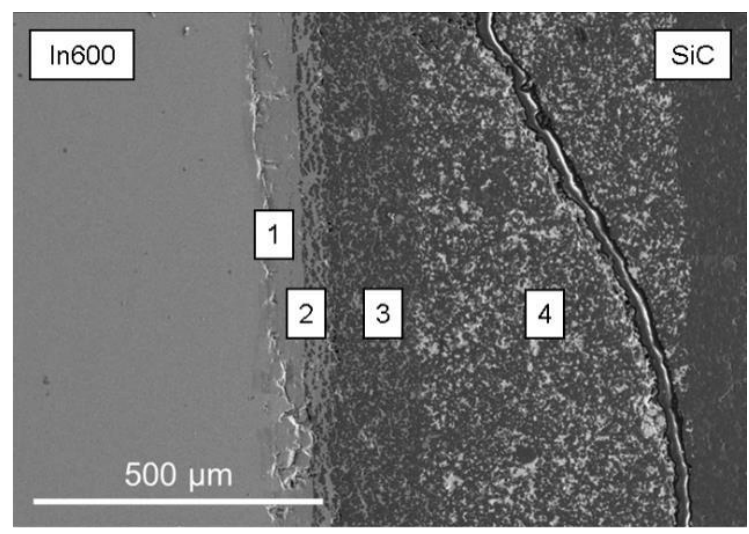

(b)

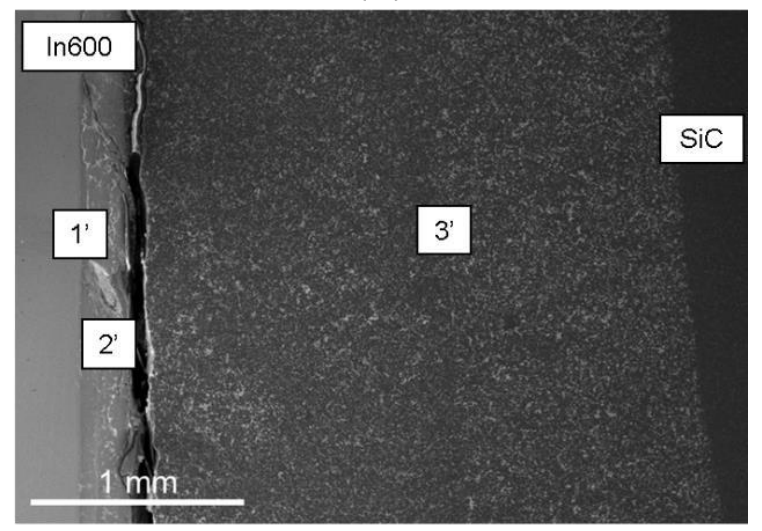

(d)

Figure 40 Secondary electron SEM cross-section images of (a) Inconel 600/Ag/SiC joint formed at $900{ }^{\circ} \mathrm{C}$ under $1 \mathrm{MPa}$ for $30 \mathrm{~min}$, (b) Inconel 600/75\% $\mathrm{Ag}$

- $25 \% \mathrm{Pd} / \mathrm{SiC}$ joint formed at $900{ }^{\circ} \mathrm{C}$ under $1 \mathrm{MPa}$ for $30 \mathrm{~min}$, (c) Inconel 600/Ag/SiC joint formed at $930{ }^{\circ} \mathrm{C}$ under $10 \mathrm{MPa}$ for $180 \mathrm{~min}$, and (d) Inconel $600 / 75 \% \mathrm{Ag}-25 \% \mathrm{Pd} / \mathrm{SiC}$ joint formed at $930{ }^{\circ} \mathrm{C}$ under $10 \mathrm{MPa}$ for $180 \mathrm{~min}$. The most relevant observation was made when comparing image (a) to (c) since the resultant microstructures observed are very different but the formation process used was the same and the only different was the presence of Pd concentration within image (c) interlayer.

\section{4: Mechanical testing}

The mechanical properties of the fabricated joints were evaluated via tensile experiments to investigate integrity of the joints. In addition, microhardness testing was used to characterize the nature of the new phases that formed at the interface and to compare them to the bulk components as well as to published literature. 


\subsection{1: Tensile Test}

Figure 41 shows the comparison in tensile strength for bonds tested as a function of the process parameters and interlayer material. For both interlayers, the joints formed at $900{ }^{\circ} \mathrm{C}$ under $1 \mathrm{MPa}$ for 30 minutes showed the highest tensile strength. While the silver had a higher average tensile strength, the error is large. The error is the standard deviation calculated from the different tensile values for each joint. Three to six total joints were tested on joints fabricated at $900{ }^{\circ} \mathrm{C}, 1 \mathrm{MPa}$, and $30 \mathrm{~min}$, joints fabricated at $900{ }^{\circ} \mathrm{C}, 10 \mathrm{MPa}$, and $30 \mathrm{~min}$, joints fabricated at $930{ }^{\circ} \mathrm{C}, 10 \mathrm{MPa}$, and $30 \mathrm{~min}$, and joints fabricated at $930{ }^{\circ} \mathrm{C}, 10 \mathrm{MPa}$, and $180 \mathrm{~min}$. The number tested varied due to pre-failure of some of the joints while setting them within the testing fixture. The principle source of error is found in the complexity and difficulty of tensile testing a ceramic component. Typically, ceramics exhibit excellent mechanical properties under compressive stress however, cracks and other flaws significantly affect the tensile behavior.

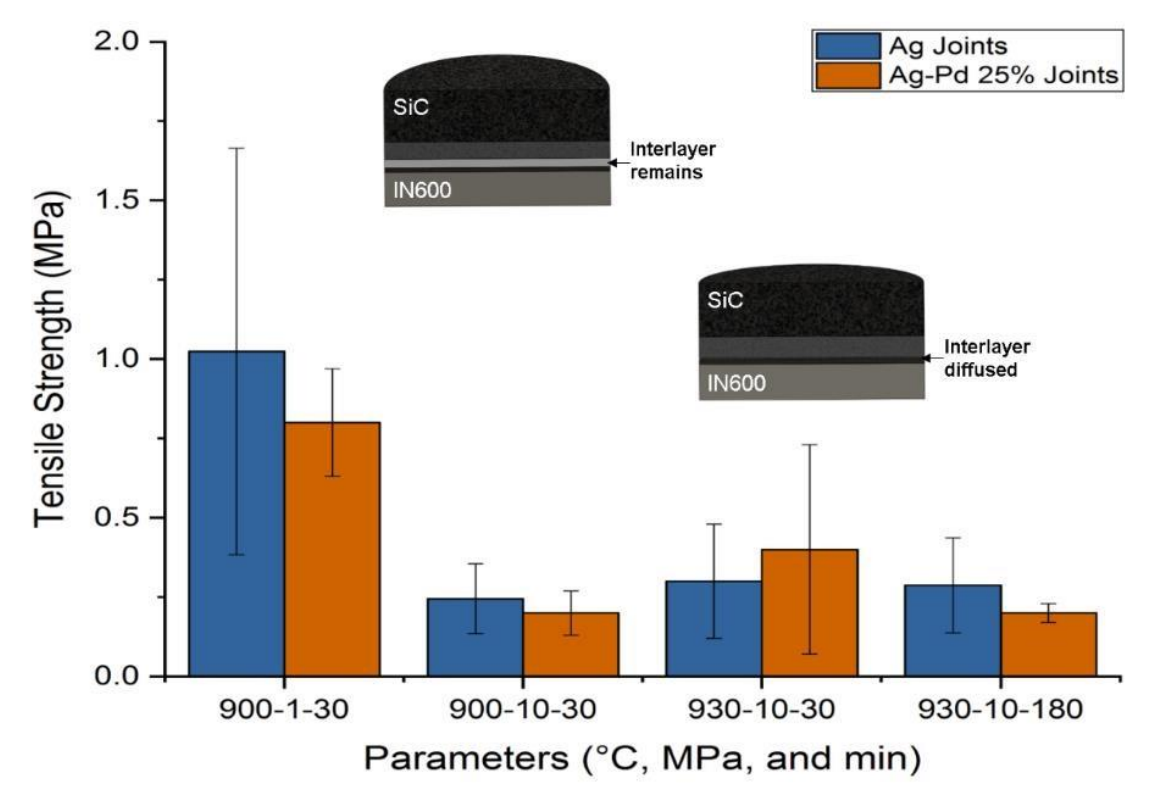

Figure 41 Tensile test results for joints fabricated with both Ag as the interlayer (blue) and Ag-Pd $25 \%$ as the interlayer (orange). 
To investigate where the fracture occurred, the fracture surfaces of the joints fabricated with the Ag interlayer were characterized with XRD to verify phases and to compliment EDS data. The XRD patterns of both fracture surfaces for joints that were tensile tested are:

- Joint formed at $900{ }^{\circ} \mathrm{C}$ under $1 \mathrm{MPa}$ for 30 minutes (Figure 42)

- Joint formed at $930{ }^{\circ} \mathrm{C}$ under $10 \mathrm{MPa}$ for 180 minutes (Figure 43)

On the fracture surfaces, multiple phases are possible, which adds complexity to the analysis. First, the largest peaks were indexed on the patterns. Secondly, the expected intermetallic phases were also considered and indexed if represented.

Figure 42 shows the $\mathrm{x}$-ray data collected from the fracture surface for the joint fabricated at $900{ }^{\circ} \mathrm{C} .1 \mathrm{MPa}$, and 30 minutes. On the pattern taken from the SiC side of the joint, XRD patterns are seen that indicate the presence of free $\mathrm{Si}, \mathrm{SiC} 4 \mathrm{H}, \mathrm{SiC} 6 \mathrm{H}$, and Ag. On the Inconel side of the joint (red pattern), only peaks associated with Ag are seen. The majority of the interlayer material remained attached to the Inconel 600 side. No minor peaks are represented or visible in the red pattern.

As the optical images of the fracture surfaces post-testing show the Ag interlayer component remains almost fully attached to the Inconel 600 side with minor amounts present within the $\mathrm{SiC}$ fracture surface side. The integrity observed of the Ag component indicates that the diffusion of the interlayer was minor. Through the optical images no necking is observed from the tensile test, and very clean smooth surfaces are observed. 


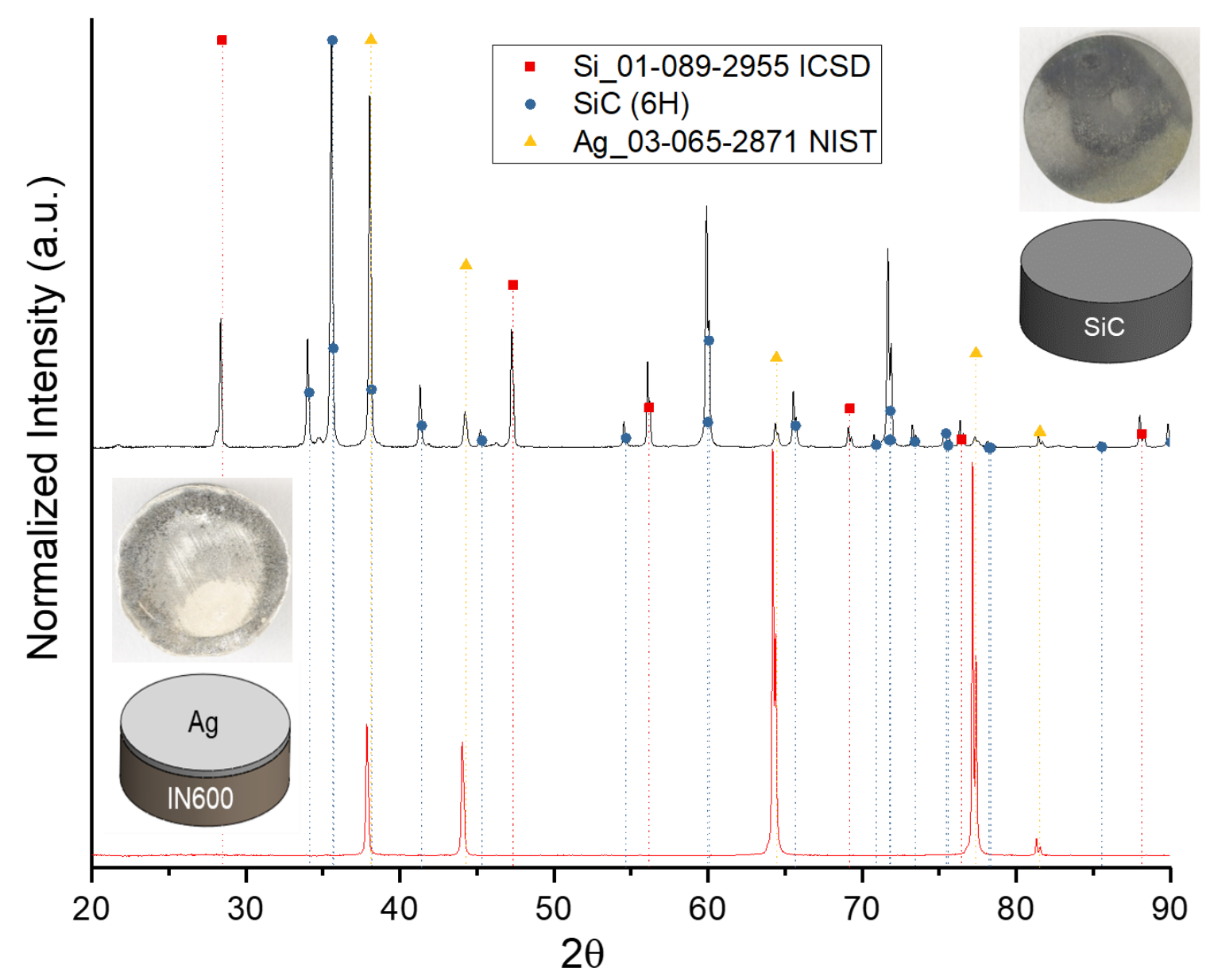

Figure 42 X-ray diffraction data of fracture surfaces of a joint formed at $900{ }^{\circ} \mathrm{C}$ under $1 \mathrm{MPa}$ for 30 minutes with the $\mathrm{Ag}$ interlayer. The top pattern is from the $\mathrm{SiC}$ fracture surface side and the bottom pattern is from the Ag-Inconel 600 side.

Figure 43 shows the $\mathrm{x}$-ray data collected from the fracture surfaces for the joint fabricated at $930{ }^{\circ} \mathrm{C} .10 \mathrm{MPa}$, and 180 minutes. Multiple phases are represented within the pattern. The phases identified include $\mathrm{Ni}$-silicide phases: $\mathrm{NiSi}, \mathrm{Ni}_{5} \mathrm{Si}_{2}$, and $\mathrm{Ni}_{3} \mathrm{Si}_{2}$. In addition, within the $\mathrm{SiC}$ fracture surface side the $\mathrm{SiC}$ and $\mathrm{Ag}$ phases are also represented within the XRD pattern, which indicates that Ag diffused into the SiC component. On the other hand, no $\mathrm{SiC}$ phases were present within the Inconel 600 fracture surface side while Ag was also observed within. 


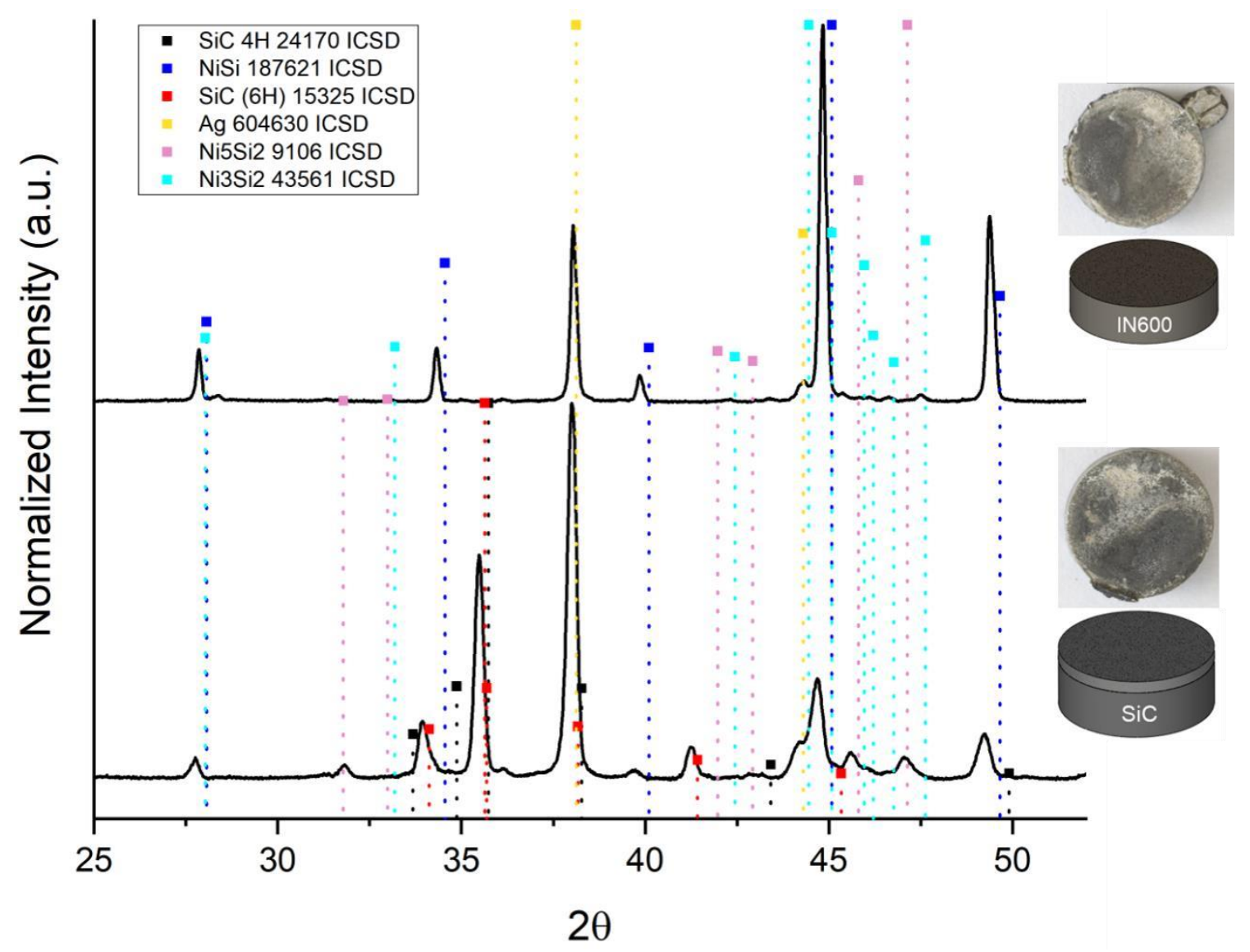

Figure $43 \quad X$-ray diffraction data of the fracture surface of the joint formed at $930{ }^{\circ} \mathrm{C}$ under $10 \mathrm{MPa}$ for 180 minutes with the $\mathrm{Ag}$ interlayer. The top pattern is from the $\mathrm{SiC}$ fracture surface side and the bottom pattern is from the Inconel 600 fracture surface side. Many phases are present within both patterns, but most prominent are $\mathrm{NiSi}, \mathrm{Ni}_{5} \mathrm{Si}_{2}$ and $\mathrm{Ni}_{3} \mathrm{Si}_{2}$.

Figure 44 shows the micrographs of the tensile tested fracture surfaces of the bonded joint at $930{ }^{\circ} \mathrm{C}$ under $10 \mathrm{MPa}$ for 180 minutes, which was used to investigate the fracture behavior of the tensile tested joints. The surface appears rough, and cleavage planes are observed. The surface is nonuniform and the different shaded regions suggest that multiple phases are present. 

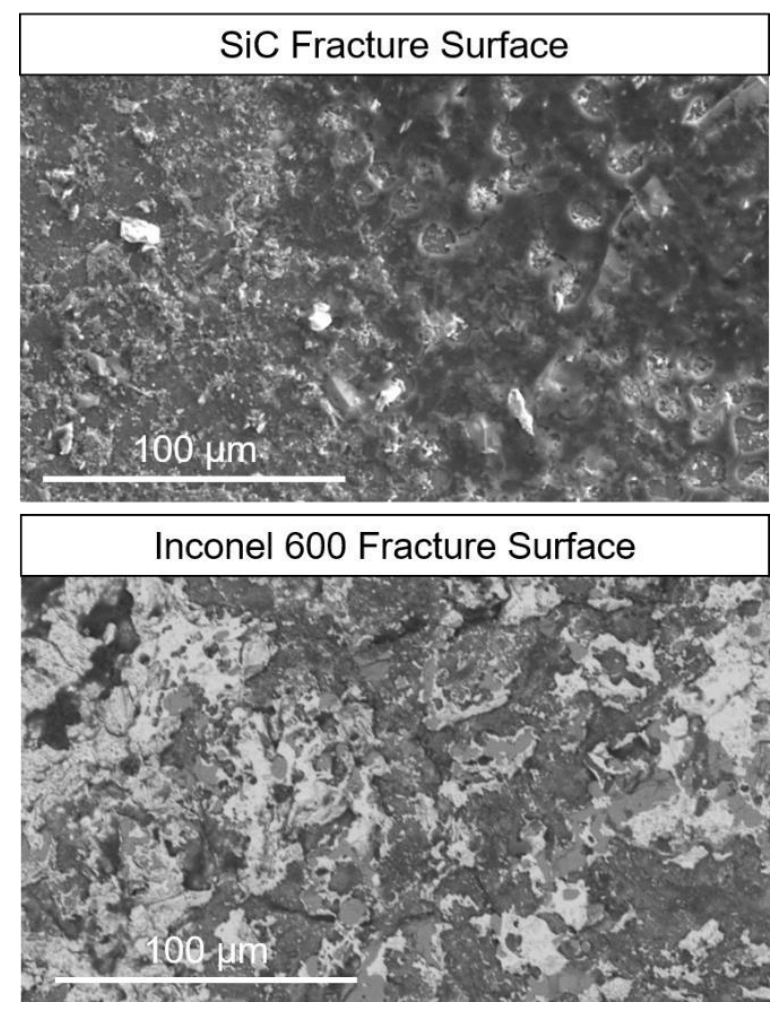

Figure 44 The tensile fracture surfaces of the joint fabricated at $930{ }^{\circ} \mathrm{C}, 10 \mathrm{MPa}$, and 30 min with Ag interlayer.

\subsection{2: Microhardness Test}

The variation in the microhardness data across the interface of the Inconel $600 / \mathrm{SiC}$ joints bonded at the different process parameter combinations are presented in Figure 45 and Figure 46. Figure 45 shows a typical SEM micrograph to indicate where hardness tests were conducted and plots the microhardness values from different regions for all joints formed with the Ag interlayer. The hardness values near the interface are larger than the hardness of either Inconel 600 or silver. Figure 46 plots the microhardness values from different regions for all joints formed with the $75 \% \mathrm{Ag}-25 \%$ Pd interlayer. Similar trends in microhardness are seen in these joints. The hardness values of the bulk components were compared to values reported in the literature. 

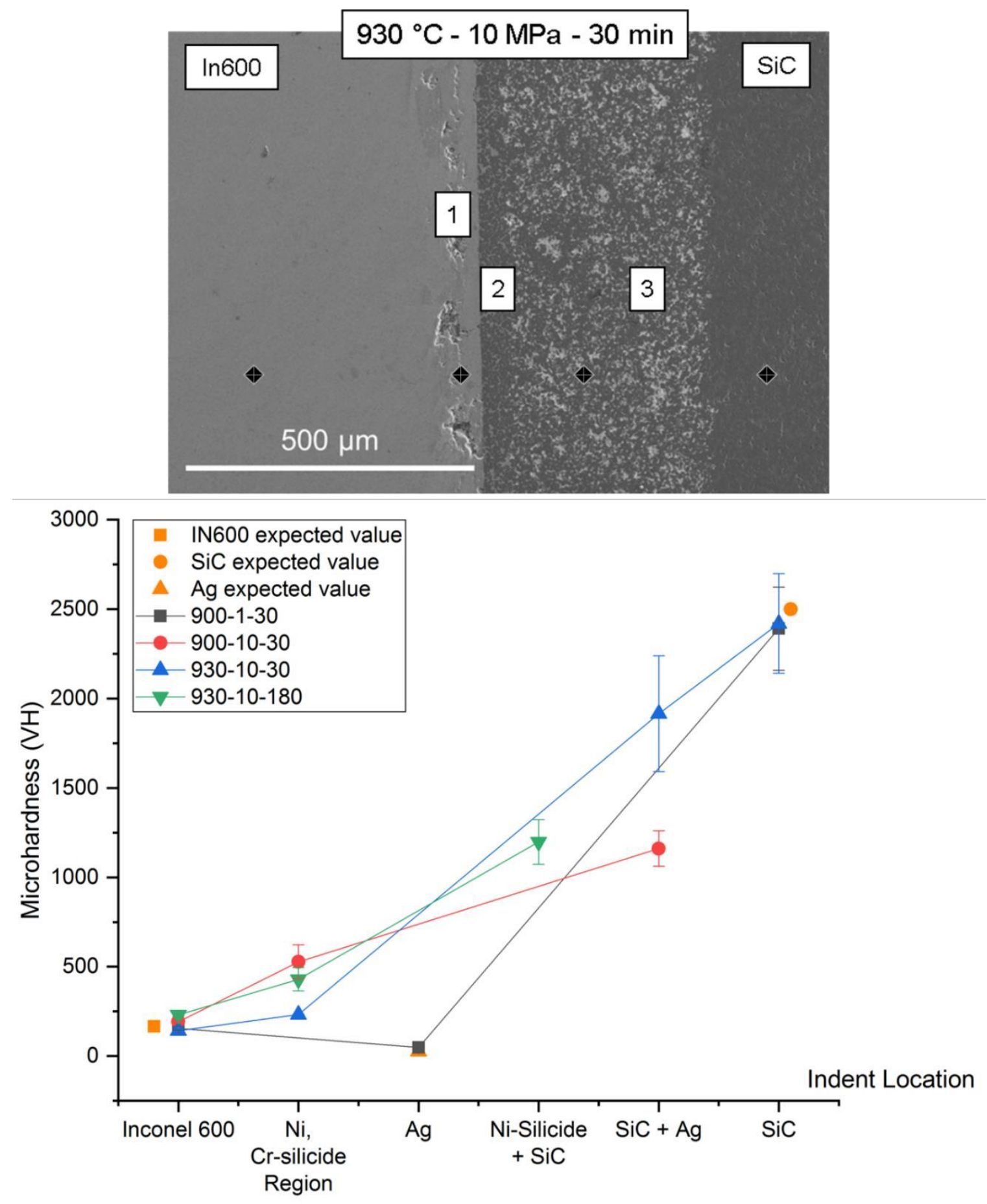

Figure 45 Microhardness data comparison for joints with Ag interlayers. The SEM image shown is from a joint fabricated at $930{ }^{\circ} \mathrm{C}, 10 \mathrm{MPa}$ and $30 \mathrm{~min}$, represented by the blue line. 

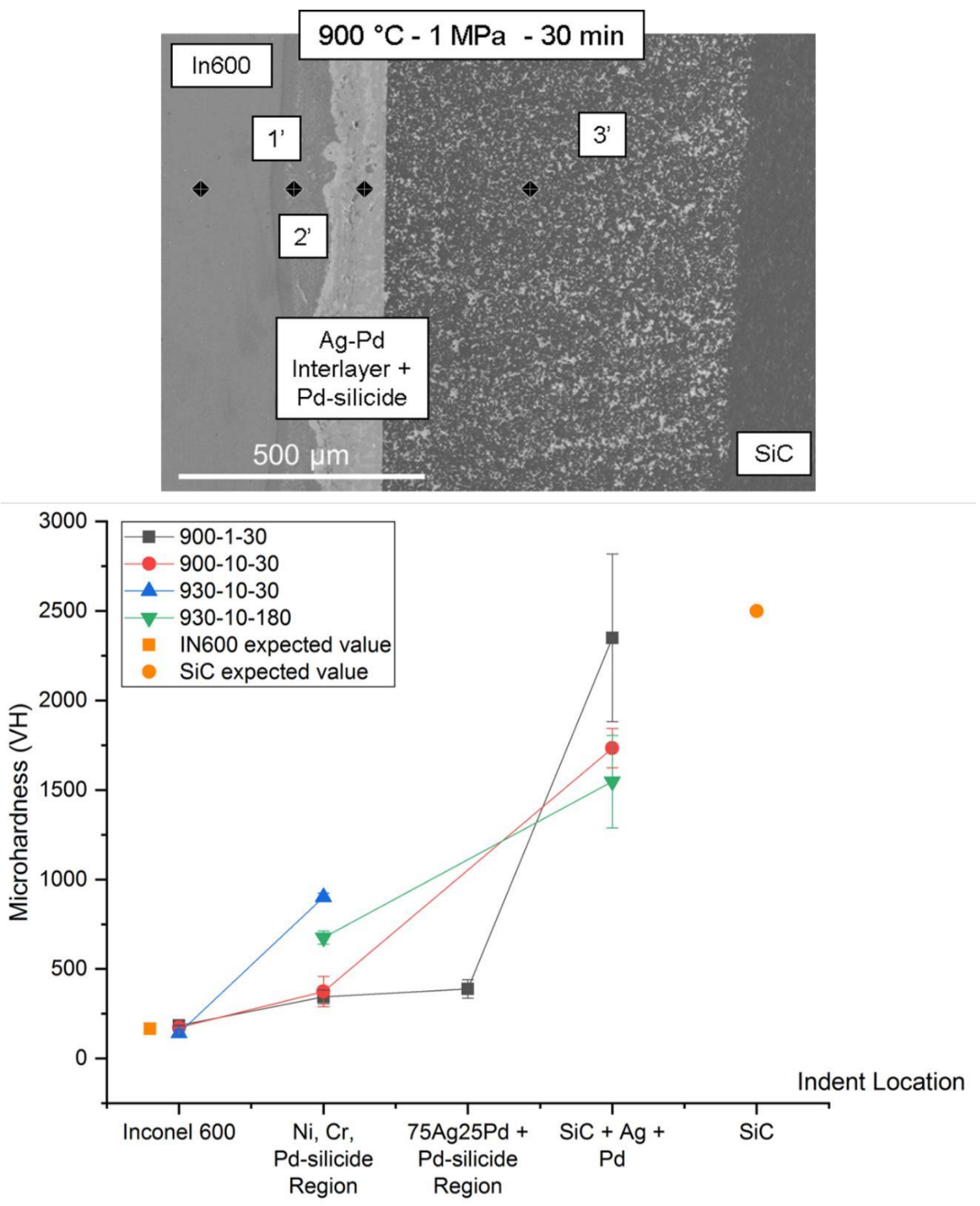

Figure 46 Microhardness data comparison for all joints with 75\% Ag-25 \% Pd interlayers on the cross-section of the joint. The SEM image shown is for a joint fabricated at $900{ }^{\circ} \mathrm{C}, 1 \mathrm{MPa}$, and $30 \mathrm{~min}$, represented by the black line. 


\section{5: Diffusion Kinetics}

To better understand the diffusion kinetics, additional bonding experiments were performed at different temperatures. The test matrix used for the kinetics investigation is shown in Table 14 . The temperature and pressure were maintained constant at $930{ }^{\circ} \mathrm{C}$ and $10 \mathrm{MPa}$, while time was increased from 30-180 min. With this test matrix, the diffusion mechanism is investigated by looking at the relationship between diffusion thickness vs. bonding time.

Table 14 The test matrix for kinetic studies for both joints formed with Ag and AgPd $25 \%$ interlayers.

\begin{tabular}{|c|c|}
\hline \multirow{3}{*}{$930{ }^{\circ} \mathrm{C}, 10 \mathrm{MPa}$} & Time (minutes) \\
\cline { 2 - 2 } & 30 \\
\cline { 2 - 2 } & 60 \\
\cline { 2 - 2 } & 120 \\
\cline { 2 - 2 } & 180 \\
\hline
\end{tabular}

The secondary electron cross-section SEM images of the interface of the fabricated joints for different bonding times were analyzed. Fiji [41] and ImageJ [42] were used to measure the reaction layer thickness of the resultant reactions regions. Figure 47 is a typical SEM image used to illustrate how the layer thicknesses were measured. In general, twenty measurements were taken per image and multiple images per joint were used, to ensure the data was representative of the overall joint. Optical images of joints fabricated with the $\mathrm{Ag}$ interlayer at $930^{\circ}, 10 \mathrm{MPa}$, and $180 \mathrm{~min}$ were used to measure six lines per images for a total of three images per joint for a total of three joints. Images were assured to be valid representation of the joint overall. Generally, edges were not considered to minimize error due to possible edge effects, because of alignment while bonding the assembly. 

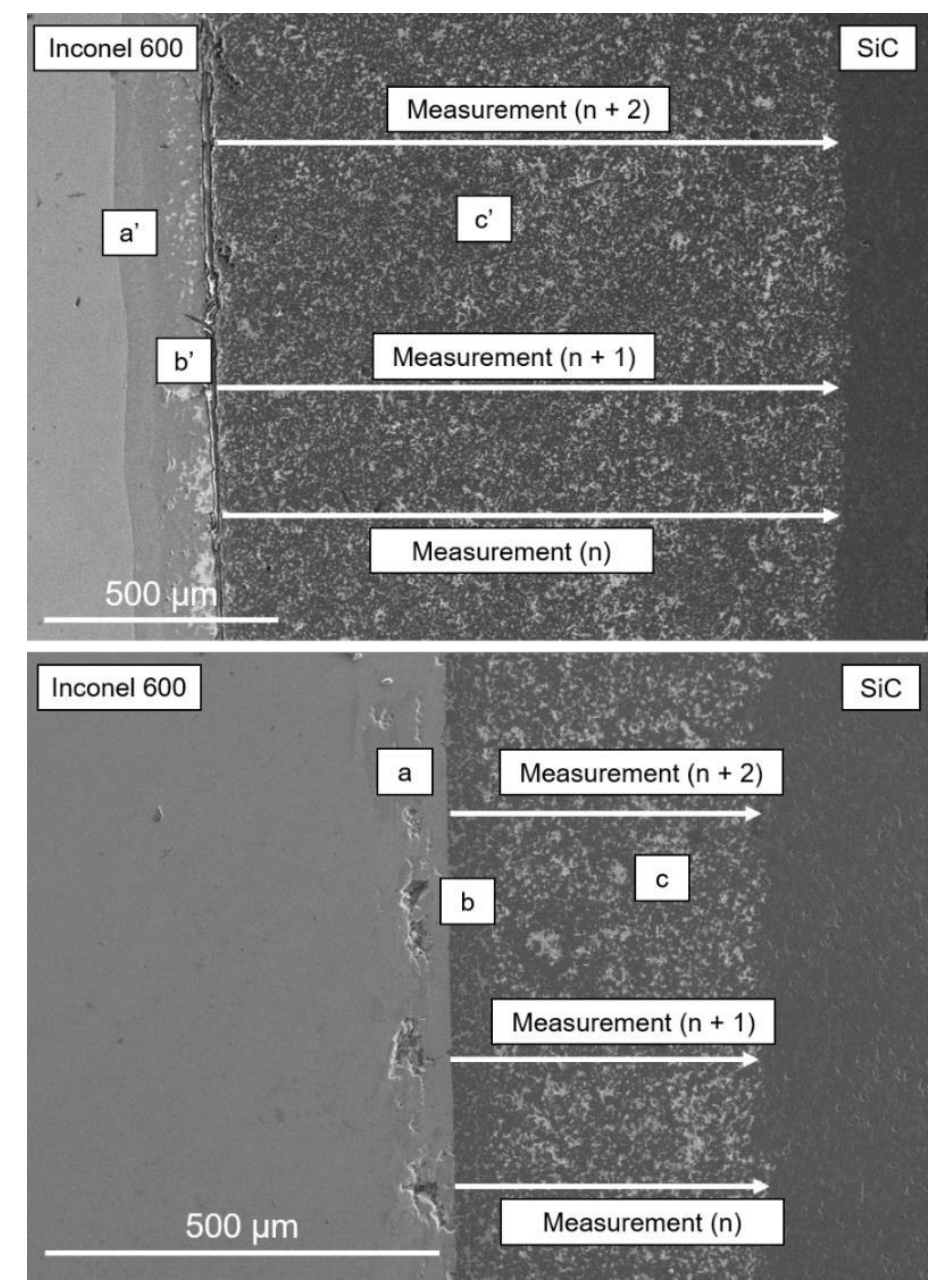

Figure 47 The SEM image of the joint fabricated in this work, showing the methodology used to measure the diffusion thickness using image software such as Fiji or ImageJ. The thickness measurements are from the Inconel 600 to $\mathrm{SiC}$ interface to where the diffusion layer c' or c end.

Table 15 and Figure 48 indicate that joints with both types of interlayers show an increase in diffusion distance as the time increases. However, from Table 15, the highlighted values deviate from the expected values. These joints were formed during the initial set of experiments. The new samples joined to better understand the effect of time on the process were created with a new $\mathrm{SiC}$ source. Hence these differences are likely due to variations in the source $\mathrm{SiC}$ material. The average thickness values for the Ag samples were taken from 6 images across the joint from 2 to 3 joints and from 18-20 measurements per image. The standard deviation values were calculated from the 
different measurements taken within joint for each bonding process used for this study.

On the other hand, the average thickness values for the Ag-Pd samples were taken from 1 image per joint since consistency from joint to joint was observed as well as from previous observations diffusion thickness across the joint was homogeneous.

Table 15 Diffusion measurements for the different formed joints presented through this study for joints formed with Ag as well as Ag-Pd 25\% interlayers.

\begin{tabular}{|c|c|c|c|c|}
\hline \multicolumn{5}{|c|}{ Ag Interlayer } \\
\hline $\begin{array}{c}\text { Temperature and } \\
\text { Pressure }\end{array}$ & Time (min) & $\begin{array}{c}\text { Average } \\
\text { Thickness }(\mu \mathrm{m}\end{array}$ & $\left.\sqrt{\text { time }} \min ^{1 / 2}\right)$ & $\begin{array}{c}\text { ST } \\
\text { DEV }\end{array}$ \\
\hline \multirow{4}{*}{$930{ }^{\circ} \mathrm{C} \& 10 \mathrm{MPa}$} & 30 & 654 & 5.5 & 27 \\
\hline & 60 & 755 & 7.7 & 22 \\
\hline & 120 & 946 & 11 & 42 \\
\hline & 180 & 487 & 13 & 39 \\
\hline $930{ }^{\circ} \mathrm{C} \& 10 \mathrm{MPa}$ & 180 & 1277 & 13 & 60 \\
\hline $930{ }^{\circ} \mathrm{C} \& 10 \mathrm{MPa}$ & 30 & 268 & 5.5 & 29 \\
\hline \multicolumn{5}{|c|}{$75 \% \mathrm{Ag}-25 \% \mathrm{Pd}$ Interlayer } \\
\hline $\begin{array}{c}\text { Temperature and } \\
\text { Pressure }\end{array}$ & Time (min) & $\begin{array}{c}\text { Average } \\
\text { Thickness }(\mu \mathrm{m}\end{array}$ & $\left.\sqrt{\text { time }(\min }{ }^{1 / 2}\right)$ & $\begin{array}{c}\text { ST } \\
\text { DEV }\end{array}$ \\
\hline \multirow{4}{*}{$930{ }^{\circ} \mathrm{C} \& 10 \mathrm{MPa}$} & 30 & 1550 & 5.5 & 20 \\
\hline & 60 & 1728 & 7.7 & 4 \\
\hline & 120 & 2160 & 11 & 16 \\
\hline & 180 & 2761 & 13 & 60 \\
\hline
\end{tabular}

Figure 48 shows a plot of the thickness of the reaction layers measured as

a function of bonding time at $930{ }^{\circ} \mathrm{C}$ and $10 \mathrm{MPa}$. Data is plotted for thickness as a function of $\sqrt{\text { time }}$. The linearity of the data indicates that the reaction layer thickness growth follows parabolic kinetics, indicating that the reaction process is diffusioncontrolled. The relationship between thickness and square root of time is given by:

$$
\mathrm{x}^{2}=\mathrm{kt}
$$


Where $\mathrm{x}$ is the measured thickness, $\mathrm{t}$ is bonding time, and $\mathrm{k}$ is the rate constant.

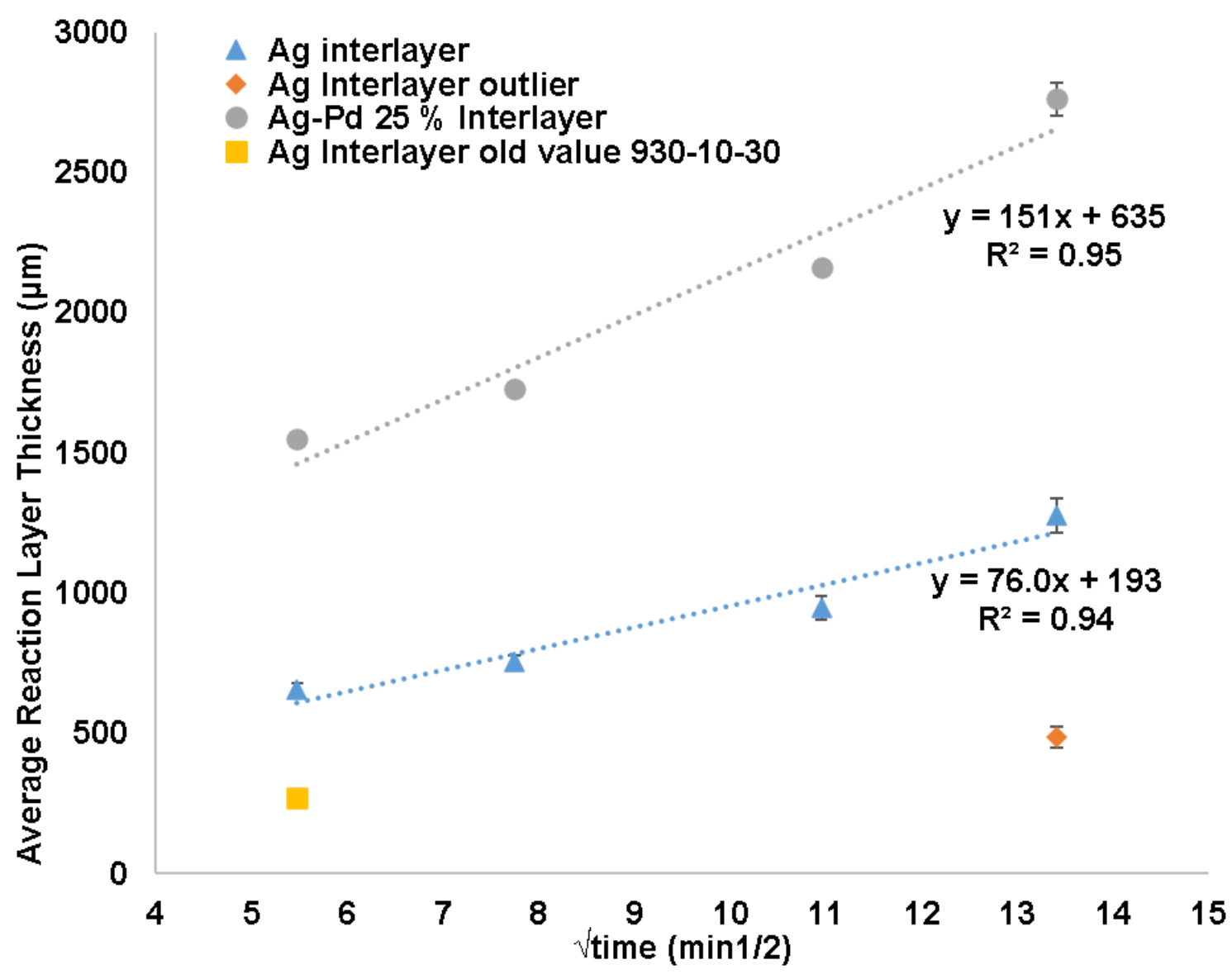

Figure 48 The plot of the diffusion layer thickness vs time ${ }^{1 / 2}$ for the $\mathrm{SiC}$ and Inconel 600 diffusion bonded transitions. Joints were formed at $930{ }^{\circ} \mathrm{C}$ under 10 MPa for times of 30, 60, 120, and 180 minutes.

The rate constant $\mathrm{k}$ for the $\mathrm{Ag}$ interlayer system was calculated to be 5630 $\mu \mathrm{m}^{2} / \mathrm{min}$ and the rate constant $\mathrm{k}$ for the $\mathrm{Ag}-\mathrm{Pd}$ interlayer system was calculated to be $22500 \mu \mathrm{m}^{2} / \mathrm{min}$, supporting the observations made about the Ag-Pd interlayer system diffusion process is accelerated and achieves larger reaction layer thicknesses. In addition, if assuming our main components are semi-infinite bars, we get the relationship [18]:

$$
\frac{x}{\sqrt{D t}}=\text { constant }
$$


$x^{2} \propto D t$

Based on the following assumption and equation (4.4), preliminary calculations for the diffusion coefficient were attempted and values are given in Table 16. However, due to the complexity of the system, it is difficult to assign the calculated D to a specific species into a specific host.

Table 16 Screen calculations of diffusion coefficient from diffusion thickness measurements and bonding times from Ag interlayer system.

\begin{tabular}{|c|c|c|c|}
\hline $\begin{array}{c}\text { Process } \\
\text { Parameters }\end{array}$ & Interlayer & $\begin{array}{c}\text { Thickness } \\
\text { overall }(\mu \mathrm{m})\end{array}$ & $\begin{array}{c}\text { Diffusion Coefficient }(\mathrm{m} / \mathrm{s}) \\
2 \\
\mathrm{D}=\mathrm{x} / \mathrm{t}\end{array}$ \\
\hline $\begin{array}{c}900{ }^{\circ} \mathrm{C} 1 \mathrm{MPa} \\
30 \mathrm{~min}\end{array}$ & $\mathrm{Ag}$ & 0 & - \\
\hline $\begin{array}{c}900{ }^{\circ} \mathrm{C} 10 \mathrm{MPa} \\
30 \mathrm{~min}\end{array}$ & $\mathrm{Ag}$ & 377 & $7.90 \mathrm{E}-11$ \\
\hline $\begin{array}{c}930{ }^{\circ} \mathrm{C} 10 \mathrm{MPa} \\
30 \mathrm{~min}\end{array}$ & $\mathrm{Ag}$ & 654 & $1.43 \mathrm{E}-8$ \\
\hline $\begin{array}{c}930{ }^{\circ} \mathrm{C} 10 \mathrm{MPa} \\
180 \mathrm{~min}\end{array}$ & $\mathrm{Ag}$ & 1277 & $9.06 \mathrm{E}-9$ \\
\hline
\end{tabular}

\section{6: Thermodynamic evaluation}

In order to aid predictions about the formation of new compounds and phases at the interface, the energy of reaction was calculated for each of the phases expected to form at the interface based on what was found in literature $[43,44]$ and using the equations 4.5 and 4.6 below:

$$
\Delta \text { Hreaction }=\text { Hformation }(\mathrm{AB})-(\text { Hformation }(\mathrm{A})+\mathrm{H} \text { formation }(\mathrm{B}))
$$


To understand the probability of phase formation, the energy of reaction is compared to the Gibbs free energy of reaction, considering the relationship for solidstate reactions:

The different $\Delta \mathrm{Hs}$ and $\Delta \mathrm{Gs}$ values extracted from Barin tables or literature for possible reactions at the interface are given in Table 17. All values are taken from reactions considered to occur at temperatures from $\mathrm{RT}$ to $950{ }^{\circ} \mathrm{C}$ since the values do not appear to differ much.

Table 17 Enthalpy of formation of nickel silicides from the reaction of $\mathrm{Ni}$ and Si. Values taken from [43-46].

\begin{tabular}{|c|c|c|c|c|c|c|}
\hline Phase & $\begin{array}{c}\Delta \mathrm{Hf} \\
(\mathrm{kJ} / \mathrm{mol}) \\
{[45]}\end{array}$ & $\begin{array}{c}\Delta \mathrm{G}_{\mathrm{f}} \\
(\mathrm{kJ} / \mathrm{mol}) \\
{[45]}\end{array}$ & $\begin{array}{c}\Delta \mathrm{H}_{\mathrm{f}} \\
(\mathrm{kJ} / \mathrm{mol}) \\
{[43]}\end{array}$ & $\begin{array}{c}\Delta \mathrm{G}_{\mathrm{f}} \\
(\mathrm{kJ} / \mathrm{mol}) \\
{[43]}\end{array}$ & $\begin{array}{c}\Delta \mathrm{H}_{\mathrm{f}}(\mathrm{kJ} / \mathrm{mol}) \\
{[44]}\end{array}$ & $\begin{array}{c}\Delta \mathrm{Hf}_{\mathrm{f}} \\
(\mathrm{kJ} / \mathrm{mol}) \\
{[46]}\end{array}$ \\
\hline $\mathrm{NiSi}$ & -88 & -81 & - & - & $-11,-16, \& 42$ & -42 \\
\hline $\mathrm{Ni}_{2} \mathrm{Si}$ & - & - & - & - & $-55,-97, \& 141$ & -47 \\
\hline $\mathrm{NiSi}_{2}$ & - & - & - & - & $-88 \&-2$ & -29 \\
\hline $\mathrm{Ni}_{5} \mathrm{Si} 2$ & - & - & - & - & -1819 & -42 \\
\hline $\mathrm{CrSi}$ & -70 & -76 & -29 & -30 & - & -30 \\
\hline $\mathrm{Cr} 3 \mathrm{Si}$ & -138 & -134 & - & - & - & -34 \\
\hline $\mathrm{CrSi} 2$ & -106 & -88 & -25 & -28 & - & -26 \\
\hline
\end{tabular}

From Table 17 discrepancy between the data extracted from Barin tables and experimental data from literature indicates the complexity of reaction formation and growth of these phases. Different sources were considered to report the values for the different possible silicide phases. The difference in source could have led to the variety of values since depending on the source, different reactions could have been considered to achieve the formation of phases. 
In addition, even within the same source depending on the chemical reaction considered to have happened that led to the formation of the silicide phase, values also differ. For example, for the formation of $\mathrm{NiSi}$ or $\mathrm{Ni}_{2} \mathrm{Si}$ the following reactions can be considered, leading onto different calculated energies:

$$
\begin{gathered}
\mathrm{Ni}+\mathrm{Si} \rightarrow \mathrm{NiSi} \\
\mathrm{Ni}_{3} \mathrm{Si}_{2}+\mathrm{Si} \rightarrow 3 \mathrm{NiSi} \\
\mathrm{Ni}_{2} \mathrm{Si}+\mathrm{Si} \rightarrow 2 \mathrm{NiSi} \\
\mathrm{NiSi}_{2}+\mathrm{Ni} \rightarrow 2 \mathrm{NiSi} \\
2 \mathrm{Ni} \mathrm{Si} \rightarrow \mathrm{Ni}_{2} \mathrm{Si} \\
\mathrm{NiSi}{ }_{2}+3 \mathrm{Ni} \rightarrow 2 \mathrm{Ni}_{2} \mathrm{Si} \\
\mathrm{NiSi}+\mathrm{Ni} \rightarrow \mathrm{Ni}_{2} \mathrm{Si}
\end{gathered}
$$

Experimental set up between sources, could have also influence the outcome of reported values between sources so for example, for $\mathrm{Ni}_{5} \mathrm{Si}_{2}$ the $\Delta \mathrm{H}_{\mathrm{f}}$ values reported by two different sources shown in Table 17 are an order of magnitude different from each other, such as that one value is $-1819 \mathrm{~kJ} / \mathrm{mol}$ and the other value is $-42 \mathrm{~kJ} / \mathrm{mol}$.

\section{CHAPTER FIVE: DISCUSSION}

The first objective of this work was to determine the effect of the process parameters on the SiC-Inconel 600 interfacial microstructure for diffusion bonded joints. 
The second objective was to investigate of the effect of the interlayer material on the interfacial solid-state reactions and overall microstructure. The third objective was to evaluate the effect of the interfacial solid-sate reactions on the mechanical integrity of the diffusion bonded joints. This chapter presents an analysis of the results presented in the previous chapters. The chapter discusses and relates the observed microstructures to the thermodynamic predictions, and to the results of other investigators. Finally, this chapter explores how the interfacial microstructure affects the mechanical properties and examines the mechanism of the interfacial reaction layer formation from the diffusion kinetics.

\section{1: Influence of Process Parameters}

When bonding dissimilar materials, processing parameters should be determined in order to optimize specific joint characteristics (e.g. joining strength, reliability, performance, etc.). These characteristics are influenced by the microstructure at the interface. In this work, microstructure characterization was used to correlate the effect of the processing parameters such as temperature, pressure and time on the stability and integrity of the joint. Characterization of the microstructure also allows understanding of the diffusion mechanism and determination of the brittle phase formation sequence.

\subsection{1: Temperature}

Although diffusion is a thermodynamically driven and kinetically controlled process, and temperature has a profound influence on the coefficients and diffusion rates, diffusing species type and bonding time also influence the process. Bonding temperatures are important as they strongly influence the diffusion and the formation of new phases. However, when bonding temperatures are too high, undesirable compounds may form 
and their morphology, distribution, and diffusion depth will influence the strength of the bonded joint bonding. Temperature influences the interfacial microstructure, and it has a significant effect on diffusion, a thermally-activated process [18].

In the case of the Ag interlayer joints, temperature was crucial to allow bonding between components. By increasing the bonding temperature, the mass transfer of the $\mathrm{Ag}$ and the free $\mathrm{Si}$ from the $\mathrm{SiC}$ component across the interface was promoted. From observations done on the cross-section SEM images of the fabricated joints coupled with the EDS map scans (Figure 32 and Figure 35), the diffusion of Ag and Si appeared to be substitutional diffusion (e.g. vacancy or interstitial diffusion). Substitutional diffusion is a diffusion mechanism in which atoms diffuse by substituting for the host atoms [47]. The $\mathrm{Ag}$ and $\mathrm{Si}$ interdiffused and it is very likely that the free $\mathrm{Si}$ in the $\mathrm{SiC}$ is the cause of this. Free $\mathrm{Si}$ exists between the $\mathrm{SiC}$ grains from the reaction bonding synthesis process of $\mathrm{SiC}$. This $\mathrm{Si}$ is free to diffuse into the Ag interlayer and the Ag diffuses into the areas where there was free Si. Given enough time and temperature, the Si will continue to diffuse into the Inconel 600 to chemically react with the elements that form the Inconel 600 composition.

The diffusion of the Si leads to formation of undesirable phases that influence the integrity and mechanical properties of the joint. The diffusion thickness of the reaction layers and phases formed during the diffusion bonding of components are mainly influenced by the bonding temperature. These observations agree with findings by G. Mahendran, et al. [48] and the study done by Junqin Li and Ping Xiao [9]. The reactivity of $\mathrm{Ag}$ was influenced by the free Si present within the SiC. Without this free Si no diffusion is believed to occur. Theoretically, the Ag should not react with SiC. A study done by Hattali, M. L., et al. [8] indicated that Ag does not interact with SiC. By 
optimizing the thickness of the interlayer, the diffusion of other reactive elements could be prevented in the $\mathrm{SiC}$.

In the case of the joints formed with the $75 \% \mathrm{Ag}-25 \% \mathrm{Pd}$ interlayers, bonding was successfully achieved at both $900{ }^{\circ} \mathrm{C}$ and $930{ }^{\circ} \mathrm{C}$. Again, from observations done on the cross-section SEM images coupled with the EDS map scans (Figure 36, Figure 37, and Figure 39), substitutional diffusion of the Ag and Pd from the interlayer material occurred into the SiC and the free Si migrated into the remaining interlayer and the Inconel 600. The diffusion of free Si caused chemical reactions within the remaining interlayer in all of the joints. The free $\mathrm{Si}$ also interacted with both the $\mathrm{Ni}$ and the $\mathrm{Cr}$ from the Inconel 600. In this set of joints, it also was observed that temperature had the highest effect on bonding, diffusion layer thickness, and formation of phases.

\subsection{2: Pressure}

Pressure is applied in forming joints in order to assure surface contact. A minimum pressure is necessary to facilitate intimate contact and allow diffusion processes to occur. The pressure needed is influenced by the temperature used for bonding [7, 48, 49]. Bonding pressure must be optimized to ensure bonding occurs. However, if the bonding pressure is much larger than needed, it can induce cracking within bulk materials or at the interface, decreasing overall bond strength.

In the case of the Ag interlayer joints, the bonding pressure was applied in order to secure a tight contact between the bonding surfaces. The observations (Figure 32) confirmed that by increasing pressure while maintaining temperature, the substitutional diffusion of $\mathrm{Ag}$ and $\mathrm{Si}$ was increased and deformation of the interlayer also increased. 
This phenomenon was observed and confirmed via EDS chemical maps. Increasing pressure results in increased surface contact and a reduction in voids, hence favorably influencing atom diffusion across the interface since more diffusion paths are created. These statements are supported by the work done by G Mahendran, et al. [48] where the author investigates the effect of bonding parameters on bonding strength, shear strength, and diffusion layer thickness. The investigation by M. Samavatian, et al. [50] evaluated the effect of pressure on diffusion bonding and concluded that as pressure was increased two phenomenon occurred. First, the bond width decreased as result of the "squeezing" action that the interlayer material experienced. Second, the surface contact increased and resulted in increased diffusion.

In the case of the $75 \% \mathrm{Ag}-25 \% \mathrm{Pd}$ interlayer joints, again, the bonding pressure was applied in order to secure a tight contact between the bonding surfaces.

Observations, (Figure 36) also confirmed that by increasing the pressure, the increased diffusion and additional reactions created new phases.

\subsection{3: Time}

The time required to form an adequate joint depends on the bonding temperature and pressure. For example, at low temperatures longer joining times may be necessary; but at high temperatures, shorter times may be enough diffusion and formation of new phases is dependent on both time and temperature. The growth of the brittle intermetallic compounds increases with bonding time. Bonding time coupled with temperature, also affects deformation although sometimes non-linearly. Once surface contact is achieved by applying pressure and plasticity increases, time is needed to fill the pores left between the local contact areas $[21,48,49]$. 
In the case of the Ag interlayer joints, the thickness of the intermetallic compounds and the diffusion region increased non-linearly with holding time, as was observed in Figure 32. Crack propagation throughout the reaction layers increased. The cracking can be attributed to the creation of additional brittle phases within the joint.

In the case of the $75 \% \mathrm{Ag}-25 \% \mathrm{Pd}$ interlayer joints, the thickness of the intermetallic compounds and the diffusion region increased with holding time linearly, as shown in Figure 36. Crack propagation also increased with increasing holding time, since more brittle phases are present. These observations agreed with the observations and conclusions in research by G. Mahendran, et al. [48] and D. Aboudi, et al. [51].

\section{2: Effects of interlayer material on the interfacial microstructure}

In addition to time, temperature and pressure, the interlayer material had a profound effect on the diffusion bonding process. Because, the influence of the process parameters on the resultant interface and joint structures is not trivial. Different interlayer materials result in different optimal parameters.

The two interlayers analyzed were $\mathrm{Ag}$ and $75 \% \mathrm{Ag}-25 \% \mathrm{Pd}$. From the results and examination of microstructures shown in Figure 40, the $25 \%$ content of Pd within the Ag interlayer catalyzes the diffusion of $\mathrm{Ag}$ into the $\mathrm{SiC}$ at both lower and higher temperatures. J. H. Neethling et al. [52] investigated the transport of $\mathrm{Ag}$ in $\mathrm{SiC}$ at $8001,000{ }^{\circ} \mathrm{C}$ and annealing times of $24-67 \mathrm{~h}$. They concluded that the transport of $\mathrm{Ag}$ in $\mathrm{SiC}$ coated particles was assisted by the presence of Pd within the fission products. Another study done by J. H. Neethling and E.J. Oliver [53] revealed that the migration of 
$\mathrm{Ag}$ in polycrystalline $\mathrm{SiC}$ can occur in association with $\mathrm{Pd}$. Other studies also suggest that Pd influences he migration of $\mathrm{Ag}$ into $\mathrm{SiC}[54,55]$.

\section{3: Effect of free $\mathrm{Si}$ in the $\mathrm{SiC}$ component}

The large amount ranging from 20-50 at\% of diffusion of Si in the Inconel 600 was reported and observed from the EDS map results and point scans (Figure 35, Figure 39, Figure 40,

Table 10, Table 11, Table 12, and Table 13). The free Si found in the SiC matrix influenced the diffusion and formation of solid-state reaction phases at the interface because of the high reactivity of $\mathrm{Si}$ with the $\mathrm{Ni}, \mathrm{Cr}$, and $\mathrm{Pd}$ elements found within the Inconel component as well as the Ag-Pd interlayer material [56, 57]. Figure 27 shows the two-phase composition of reaction bonded SiC. The comparison of interfacial microstructure shown in Figure 32 and Figure 36, the preliminary calculated diffusion coefficients given in Table 16, coupled with the EDS map scans shown in Figure 35, Figure 36, and Figure 37, and diffusivity constant extrapolated from Figure 8 in literature [18] suggest that the diffusion of $\mathrm{Ag}$ into the $\mathrm{SiC}$ component is substitutional diffusion.

The free Si also diffuses into the Inconel 600 component. A similar study by M.L. Hattali et al. [8] used pure SiC and no diffusion of Si was detected. These results suggest that the diffusion of $\mathrm{Ag}$ through the $\mathrm{SiC}$ matrix is facilitated by the free $\mathrm{Si}$ present within the $\mathrm{SiC}$ component. This statement agrees with the studies done by [58] as well as with the conclusion stated by the authors from [59], that studied the silver diffusion coefficient in single crystalline $\mathrm{SiC}$ and found that it was "extremely" low up to $1600{ }^{\circ} \mathrm{C}$ and stated the following: "The fact that significantly higher effective diffusion coefficients have been determined in silver release studies must therefore be either due to $\mathrm{SiC}$ coating containing 
diffusion enhancing impurities in their grain boundaries or structural imperfections like cracks or pores." [59].

\section{4: Interfacial Microstructure Interpretation}

A cross-section of each joint was characterized via scanning electron microscopy (SEM) and energy dispersive X-ray spectroscopy (EDS) analysis. The mechanical properties and integrity of the bonded joints are primarily influenced by the thickness of the intermetallic compounds, which is affected by the bonding temperature. The interfacial composition was different between the two interlayer materials (Ag and 75\% $\mathrm{Ag}-25 \% \mathrm{Pd}$ ) and had a different composition than the three base materials. Phase diagrams [60] of simple binary systems are shown in Figure 50 and were coupled with literature to validate the labeling of the phase formation sequence shown in Figure 51 and Figure 52. To understand the reactions that occur in the interfacial layer, diffusion is considered from a theoretical point of view using diffusion couples and considering the results from literature on the diffusion couples of the materials of interest $[18,23,56$, 6170].

In addition, in order to understand the formation of new compounds at the interface, thermodynamic concepts were considered such as, the energy of formation of the phases expected to form at the interface known as enthalpy $(\mathrm{H})$. The $\Delta \mathrm{H}$ of reaction refers to the theoretical heat released (exothermic) or absorbed (endothermic) during the process. Also, Gibbs free energy $(\mathrm{G})$, which defines the thermodynamic stability of a phase and likeliness of formation was considered $[18,70]$. 
To investigate the reaction mechanism happening within this study, a Second Order Bimolecular reaction is considered from a theoretical point of view [71]. This reaction is a second order overall but is a first order in each of the reactants involved.

$$
A+B \text { products:o: }(A B)
$$

Equation (5.1) represents the change in the enthalpy of reaction, meaning the energy required for a reaction to happen.

$$
\Delta H_{\text {reaction }}=\Delta H_{\text {formation }}(\text { products })-\Delta H_{\text {formation }}(\text { reactants })
$$

Equation (5.2) represents the same energy of reaction but expanded to consider the different components that are involved in the reaction.

$$
\Delta H_{\text {reaction }}=H_{\text {formation }}(A B)-\left(H_{\text {formation }}(A)+H_{\text {formation }}(B)\right)
$$

If the $\Delta \mathrm{H}_{\text {reaction }}<0$, the reaction is exothermic and energy is released as heat, however if the $\Delta \mathrm{H}_{\text {reaction }}>0$, the reaction is endothermic and absorbs heat.

The driving force or energy required or available for a process to take place is given by the change in the Gibbs free energy described by equation (5.3) which determines the spontaneity and likelihood of the reaction:

$$
\Delta \mathrm{G}_{\mathrm{f}}=\Delta \mathrm{H}_{\mathrm{f}}-\mathrm{T} \Delta \mathrm{S}_{\mathrm{f}}
$$

Where $\Delta \mathrm{H}_{\mathrm{f}}$ is the change in Enthalpy of formation for a specific reaction to occur, $\mathrm{T}$ is the temperature at what reactions/formation takes place, $\Delta \mathrm{S}_{\mathrm{f}}$ is the change in Entropy of formation, and $\Delta \mathrm{G}_{\mathrm{f}}$ is the change in Gibbs free energy of formation. 
For simplicity and because all materials are in the solid form, an assumption of $\Delta \mathrm{S}_{\mathrm{f}}=0$ was made. Therefore the $\mathrm{T} \Delta \mathrm{S}_{\mathrm{f}}$ component in equation (5.4) is 0 and then the change in the enthalpy of formation is approximately equal to the change in Gibbs free energy of formation $[44,46]$.

$$
\Delta \mathrm{G}_{\mathrm{f}}
$$

Due to the complexity observed through the thermodynamic evaluation (Table 16) it is to be believed that the formation of multiple metal-silicide phases exist at the interface. The solid-state reaction phases were predicted by considering literature as well. Results in studies by R. C. J. Schiepers, et al. [67], Junqin Li and Ping Xiao [9], Zhiqin Wen, et al. [72], and K. Bhanumurthy, R. Schimid-Fetzer [56] show the formation of the following phases in similar systems: $\mathrm{NiSi}_{2}, \mathrm{Ni}_{2} \mathrm{Si}, \mathrm{Ni}_{5} \mathrm{Si}_{2}, \mathrm{Cr}_{3} \mathrm{Si}, \mathrm{CrSi}, \mathrm{CrSi}_{2}, \mathrm{Pd}_{2} \mathrm{Si}$, $\mathrm{Pd}_{3} \mathrm{Si}$, and $\mathrm{Ni}_{5} \mathrm{Cr}_{3} \mathrm{Si}_{2}$. The crystal structures of these materials are shown in Figure 49. Other investigations by L. A. Clevenger, et al. [64], J. C. Feng, et al. [73], C. Lavoie, et al. [65], M. Backhaus-Ricoult [61], and J. S. Park, et al. [74] found that Ni-, Fe-, Cr- Si reaction products formed due to the high reactivity of Si with the transition metals. In addition, some of these papers $[61,64,65,73,74]$, investigated the growth kinetics during diffusion bonding, concluding that four kinds of phases were formed; $\mathrm{Ni}_{2} \mathrm{Si}$, hexagonal graphite, $\mathrm{Ni}_{5} \mathrm{Cr}_{3} \mathrm{Si}_{2}$ and $\mathrm{Cr}_{3} \mathrm{Ni}_{2} \mathrm{SiC}$. Some of these phases are also observed in this study, as shown from the formation sequence shown in both Figure 51 and Figure 52.

In summary, coupling observations made from EDS map scans, ratios from EDS point scans, phases identified via XRD, literature expectations, phase diagrams, and considering the most negative $\Delta \mathrm{G}_{\mathrm{f}}$ indicating the likeliness of formation of phases as well 
as its chemical stability once thermodynamic equilibrium is assumed, the phases expected to have formed and be present at the interface of the two interlayer materials systems are shown in color-coded form in Figure 51and Figure 52.
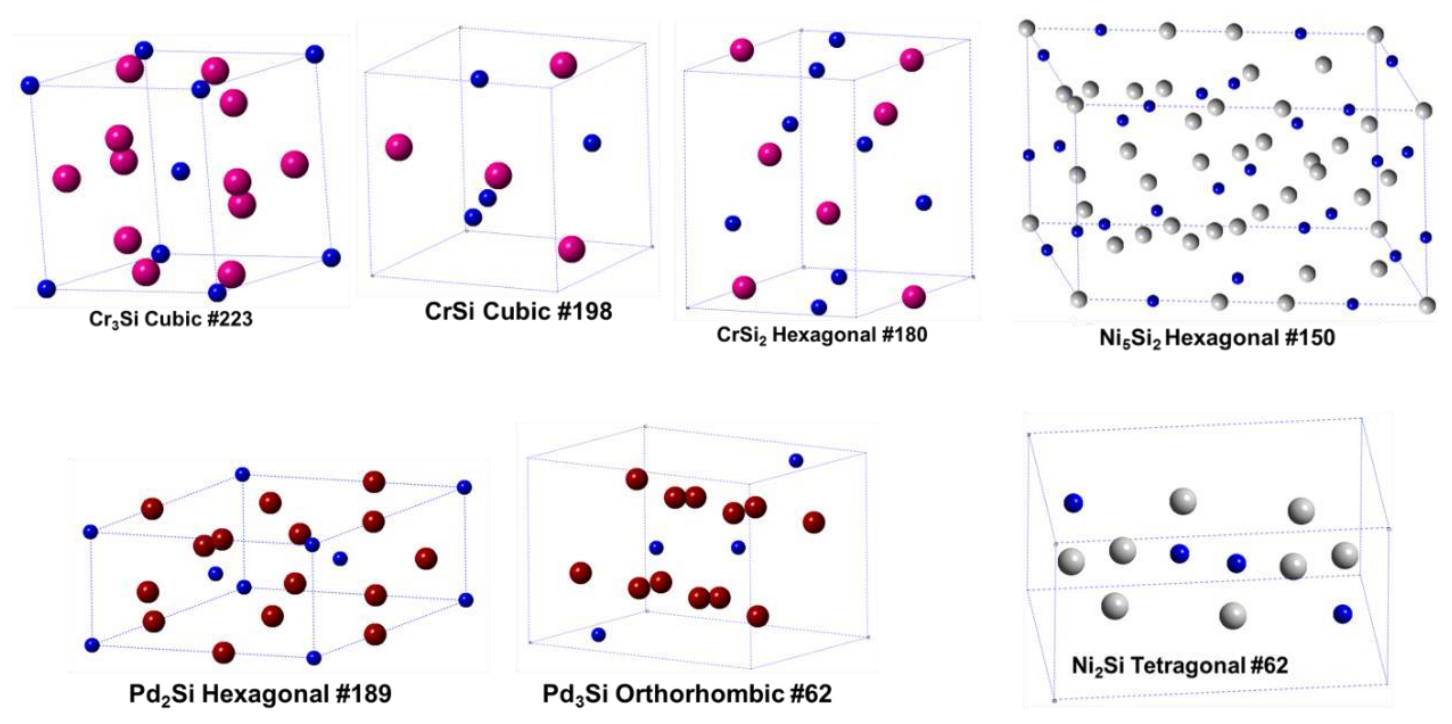

Figure 49 Crystal structure of possible interfacial silicide compounds from the diffusion bonding of $\mathrm{SiC}$ to Inconel 600 . Structures modeled with CrystalMaker. 

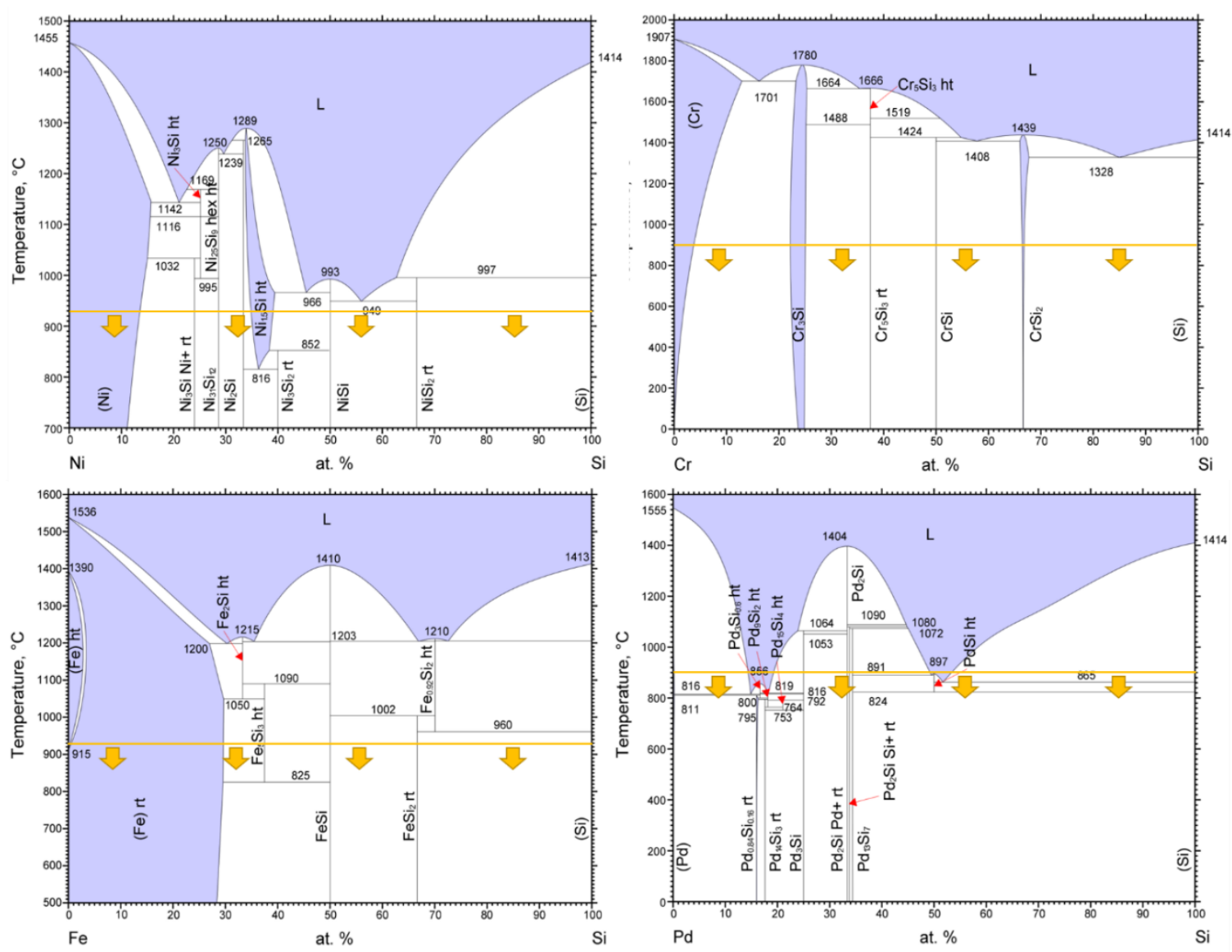

Figure 50 Phase diagrams of diffusion couples considered when indexing phases based on concentration ratios from the EDS point scans [60].

\subsection{1: Microstructural Reactions on Ag interlayer system}

The interfacial solid-state reaction formation of layers from the bonded Inconel 600-Ag-SiC are graphically described below. The phases from the joint formed at $930{ }^{\circ} \mathrm{C}$ under $10 \mathrm{MPa}$ for 180 minutes with the $\mathrm{Ag}$ as interlayer are shown in Table 18. These phases were confirmed via XRD and the chemical composition of the phases were confirmed via EDS point scans. In addition, the predicted phases were compared to literature, diffusion couple results, thermodynamics data, XRD indexed peaks, and the phase diagrams shown in Figure 50.

Table 18 Expected phases to possibly form at the Inconel 600/SiC interface of joint formed at $930{ }^{\circ} \mathrm{C}, 10 \mathrm{MPa}, 180 \mathrm{~min}$ (Figure 35), due to the diffusion of free $\mathrm{Si}$ into the metal component and the reaction of this $\mathrm{Si}$ with the base elements from the 


\section{Inconel component such as $\mathrm{Ni}$ and $\mathrm{Cr}$, based on combined SEM/EDS/XRD/Literature data.}

\begin{tabular}{|c|c|}
\hline Point & Phase \\
\hline 1 & Inconel 600 \\
\hline 2 & Fe in Ni2CrSi \\
\hline 3 & $\mathrm{Cr} 3 \mathrm{Si}$ \\
\hline 4 & $\mathrm{Ag}$ rich \\
\hline 5 and 6 & $\mathrm{Cr}$ and Fe in $\left(\mathrm{Ni3Si2}+\mathrm{Ni}+\mathrm{NiSi}\right.$ or $\left.+\mathrm{Ni}_{2} \mathrm{Si}\right)$ \\
\hline 7 & $\mathrm{SiC}$ \\
\hline
\end{tabular}

Figure 51 shows the formation sequence of the reaction layers believed to occur

during the bonding experiments. The number and amount of phases present in the joint changes as a result of the process parameters. The reaction regions grow as temperature, pressure or time is increased. The phases were predicted by considering all the results collected from observations made by analyzing the EDS map scans to predicted phases base on the ratios measured via EDS point scans to compare the predictions to the phases characterized via XRD from the fracture surfaces of Ag interlayer joints, in addition to the likeliness of formation already evaluated by comparing the Gibbs free energy of formation of the possible different phases either using thermodynamic data from Barin tables or from published studies. To finally, compared the prediction to literature expectations as well as to what phases are expected when considering phase diagrams of the different couples Ni-Si, Cr-Si, and Fe-Si presented in Figure 50 above. 


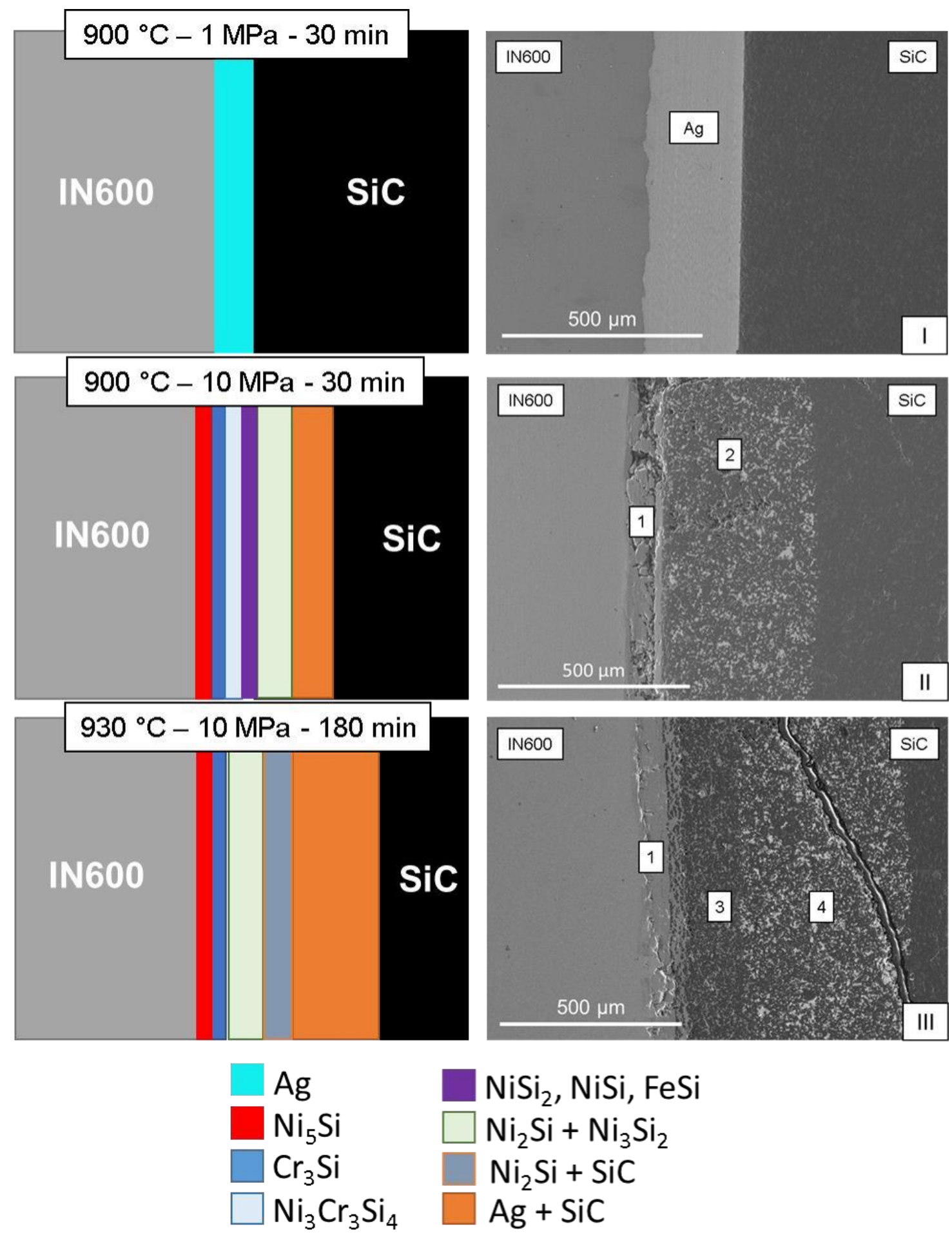

Figure 51 The formation sequence of reaction layers at the interface of joints fabricated at different combinations of process parameters. Each reaction layer is color-coded to correspond to a composition from the EDS point scans. The composition of the phases is determined by the concentration ratio of each element present in the regions and the expectations from a phase diagram plot. 


\subsection{2: Microstructure Results on the $\operatorname{Ag}_{75} \mathrm{Pd}_{25}$ interlayer system}

The interfacial solid-state reaction formation sequence from the bonded Inconel $600-\mathrm{Ag}_{75} \mathrm{Pd}_{25}-\mathrm{SiC}$ are different than that of the pure $\mathrm{Ag}$ layer. The phases present in the joint formed at $900^{\circ} \mathrm{C} 1 \mathrm{MPa}$ for 30 minutes are given in Table 19. Similarly, to Ag interlayer interfacial solid-state phases, the phases expected and predicted to have formed at the interface of the formed Ag-Pd interlayer joints were predominantly Ni-silicides with the addition of $\mathrm{CrSi} 2$ and $\mathrm{Pd}$-silicide phases since $\mathrm{Pd}$ also is highly reactive with $\mathrm{Si}$. A summary of the evolution of phase formation as process parameters increased is shown in color-coded style in Figure 52. The prediction of phases has always uncertainty since due to sample size repeatability has not been proven as well as further characterization isolating region at a time or using a higher resolution tool. The complexity of the system as can be observed through this document as well as the scattered also present within the thermodynamic data shown in literature limits the accuracy of prediction on the phases that form at the interface of these metal/ceramic transitions.

Table 20 shows the phases present in the joint fabricated at $930^{\circ} \mathrm{C} 10 \mathrm{MPa}$ for 180 minutes. The phases were confirmed from the concentration ratios collected via the EDS point scans and compared to literature, diffusion couple results, thermodynamics data, and the phase diagrams shown in Figure 50. Similarly, to Ag interlayer interfacial solidstate phases, the phases expected and predicted to have formed at the interface of the formed $\mathrm{Ag}-\mathrm{Pd}$ interlayer joints were predominantly $\mathrm{Ni}$-silicides with the addition of $\mathrm{CrSi}_{2}$ and Pd-silicide phases since Pd also is highly reactive with Si. A summary of the evolution of phase formation as process parameters increased is shown in color-coded 
style in Figure 52. The prediction of phases has always uncertainty since due to sample size repeatability has not been proven as well as further characterization isolating region at a time or using a higher resolution tool. The complexity of the system as can be observed through this document as well as the scattered also present within the thermodynamic data shown in literature limits the accuracy of prediction on the phases that form at the interface of these metal/ceramic transitions.

Figure 52 below, shows the extrapolated formation sequence within two interfacial microstructures of joints fabricated at $900^{\circ} \mathrm{C}, 1 \mathrm{MPa}$, and $30 \mathrm{~min}$ and $930^{\circ} \mathrm{C}$, $10 \mathrm{MPa}$, and $180 \mathrm{~min}$. Phases are supported by the EDS map scans, EDS line scan, and EDS point scans presented in Figure 37, Figure 38, Figure 39, and collected on Table 19 and Table 20. Following the same approved as the one used for the Ag interlayer system, the phases were predicted by considering all the results collected from observations made by analyzing the EDS map scans, EDS line scan, to predicted phases base on the ratios measured via EDS point scans to compare the predictions to the likeliness of formation already evaluated by comparing the Gibbs free energy of formation of the possible different phases either using thermodynamic data from Barin tables or from published studies. To in the end, compared the prediction to literature expectations as well as to what phases are expected when considering phase diagrams of the different couples $\mathrm{Ni}$ Si, Cr-Si, Pd-Si and Fe-Si presented in Figure 50 also. 

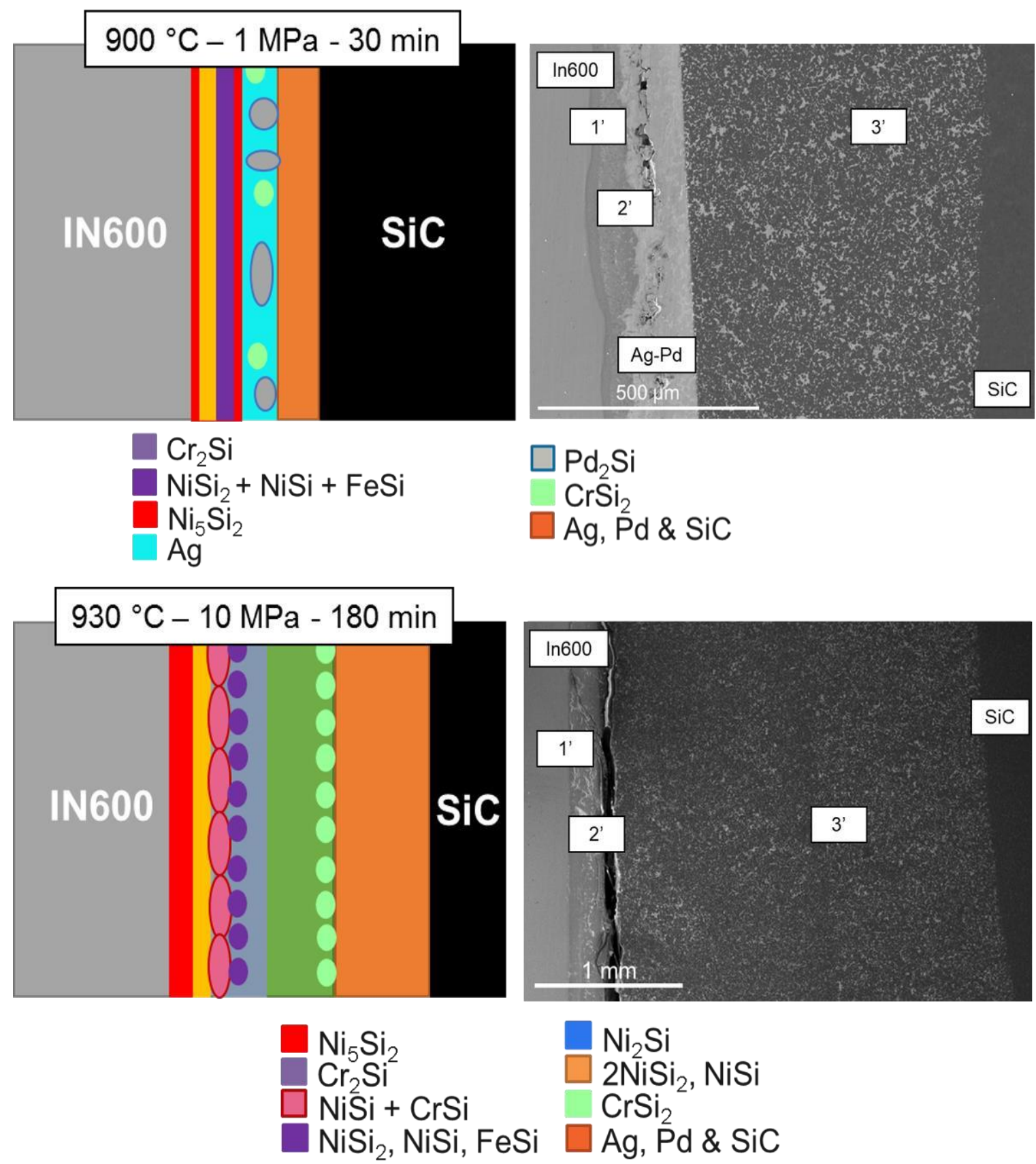

$\mathrm{Pd}_{2} \mathrm{Si}$

$\mathrm{CrSi}_{2}$

$\mathrm{Ag}, \mathrm{Pd} \& \mathrm{SiC}$

$\mathrm{Ni}_{2} \mathrm{Si}$

$2 \mathrm{NiSi}_{2}, \mathrm{NiSi}$

$\mathrm{CrSi}_{2}$

$\mathrm{Ag}, \mathrm{Pd} \& \mathrm{SiC}$

Figure 52 The formation sequence of reaction layers at the interface of joints fabricated at $900{ }^{\circ} \mathrm{C}$ under $1 \mathrm{MPa}$ for $30 \mathrm{~min}$ and at $930{ }^{\circ} \mathrm{C}$ under $10 \mathrm{MPa}$ for 180 min. Each reaction region is color-coded and relates to a specific concentration from the EDS point scans taken. The ratio extrapolated from these concentrations suggested the identification of specific phases.

Table 19 Phases that formed at the Inconel 600/SiC interface of the joint formed at $900{ }^{\circ} \mathrm{C}, 1 \mathrm{MPa}, 30 \mathrm{~min}$ (Figure 37) 


\begin{tabular}{|c|c|}
\hline 1 & Inconel 600 \\
\hline 2 & $\mathrm{Cr}_{2} \mathrm{Si}$ \\
\hline 3 & $\mathrm{NiSi}_{2}+\mathrm{NiSi}+\mathrm{FeSi}$ \\
\hline 4 & $\mathrm{Ag}$ \\
\hline 5 & $\mathrm{Pd}_{2} \mathrm{Si}$ \\
\hline 6 & $\mathrm{CrSi}_{2}$ \\
\hline
\end{tabular}

Table 20 Phases that formed at the Inconel 600/SiC interface of the joint bonded at $930{ }^{\circ} \mathrm{C}, 10 \mathrm{MPa}, 180 \mathrm{~min}$ (Figure 39)

\begin{tabular}{|c|c|}
\hline Point & Phase \\
\hline 1 & Inconel 600 \\
\hline 2 & $\mathrm{Ni}_{5} \mathrm{Si} 2$ \\
\hline 3 & $\mathrm{Cr}_{2} \mathrm{Si}$ \\
\hline 4 & $\mathrm{NiSi}+\mathrm{CrSi}$ \\
\hline 5 & $\mathrm{NiSi}_{2}+\mathrm{NiSi}+\mathrm{FeSi}$ \\
\hline 6 & $\mathrm{Ni}_{2} \mathrm{Si}$ \\
\hline 7 & $2 \mathrm{NiSi}_{2}+\mathrm{NiSi}$ \\
\hline 8 & $\mathrm{Ag}$ \\
\hline 9 & $\mathrm{CrSi}_{2}$ \\
\hline
\end{tabular}

\subsection{3: Interface Thermal Cracking Analysis}

For both interlayer systems, minor and major cracking phenomenon were

observed in many of the interfacial regions. SEM images suggest that the cracking originated and propagated as a result of a combination of thermal stresses from the CTE mismatch and the formation of brittle intermetallic phases, which influence the microstructural properties. Similar behavior was seen in other studies $[3,21,25]$. As shown in Figure 10, cracking propagates perpendicular to the interface if cracking is due to a mismatch in the coefficient of thermal expansion of the bonded materials. On the other hand, if cracking propagates along the interface, this phenomenon is explained by the contraction expected to be experienced and produced by the formation of brittle phases $[3,21,75]$. 


\section{5: Mechanical Properties}

\subsection{1: Tensile Test}

The highest tensile strength was seen in joints fabricated at $900{ }^{\circ} \mathrm{C}, 1 \mathrm{MPa}$, and 30 minutes. The observations from Figure 41 led to the conclusion that the highest tensile strength was achieved as result of maintaining the integrity of the ductile interlayer at the interface. The purpose of the interlayer is to minimize the effect of residual stresses caused by the CTE mismatch. However, if the interlayer diffuses into the ceramic component, the structure loses its buffer component introduced to account for the CTE mismatch expected to exist between the SiC and the Inconel 600.

Although the highest strength was achieved in the joints fabricated at $900{ }^{\circ} \mathrm{C}, 1$ MPa and $30 \mathrm{~min}$, these joints also showed the highest variation in strength. In agreement with the study done by A.K. Jadoon et al. [25], failure in diffusion bonded joints was mainly attributed to thermal stresses and the formation of brittle interfacial phases. Hence, the high strength in the $900{ }^{\circ} \mathrm{C}, 1 \mathrm{MPa}$ and $30 \mathrm{~min}$ joint is likely because the ductile Ag interlayer was still present and the formation of new phases was not seen. The variation in the strength of the joint can be accounted for by variation in the number of interfacial defects and local variation of stresses due to the geometry of the joint. The mechanical properties of the metal-ceramic interface and the loading conditions also influenced the joint failure. The scatter in the data may also have been impacted by the brittle behavior expected from SiC under tension loads, as well cracking introduced in SiC during heating and cooling.

The failure mechanism was expected to be brittle as the fabrication parameters were increased, since more brittle phases were formed. This expectation and results were in contradiction to the study done by Chao Xhang, et al. [76] that concluded that as the 
bond strength increases, the failure mechanism becomes more ductile, represented by the concavities and surface texture shown in the Figures 7 and 8 presented in Chao's paper.

The fracture surfaces of the $900{ }^{\circ} \mathrm{C}, 1 \mathrm{MPa}$, and $30 \mathrm{~min}$ and the $930^{\circ} \mathrm{C}, 10 \mathrm{MPa}$, and 180 min samples were analyzed by XRD after tensile testing. The failure mechanism observed from the fracture surface analysis on all joints except those fabricated at $900{ }^{\circ} \mathrm{C}$, $1 \mathrm{MPa}$, and 30 min was governed by brittle behavior, as would be expected considering that brittle phases formed at the interface where the fracture occurred. The brittle phases identified by XRD were shown in Figure 43. The surface fractography shown in the Figure $44(100 \mu \mathrm{m})$ images for the $\mathrm{SiC}$ and Inconel 600 side, conclude that the crack propagation occurred through the interior of the grains, indicating transgranular fracture. For most brittle crystalline materials, crack propagation is due to the repeated breaking of atomic bonds along specific crystallographic planes [18]. This process is known as cleavage. This type of fracture is said to be transgranular because the fracture cracks pass through the grains. Brittle fracture takes place without any appreciable deformation and by rapid crack propagation. The direction of crack motion is perpendicular to the applied tensile stress direction and results in a relatively flat fracture surface [18].

The tensile strength values collected in MPa were small due to the complexity of the geometry and the sample material being tensile tested, as well as the size of the sample and the presence of impurities and cracks already in the structure prior to setting up, which required very minimum contact to lead to fracture. 


\subsection{2: Microhardness Test}

By comparing the hardness values at multiple points across the interface of the joints and comparing these to the values of the as-received $\mathrm{SiC}$ and Inconel 600, new intermetallic brittle phases forming at the interface were identified. The variation in the microhardness values across the interface of Inconel $600 / \mathrm{SiC}$ joints with $\mathrm{Ag}$ interlayers is shown in Figure 45. The similar data for the joints fabricated with interlayer $\mathrm{Ag}_{75} \mathrm{Pd}_{25}$ are shown in Figure 46. These two plots show that the hardness values near the interface increased with respect to the bulk hardness values for both the as-received Inconel 600 and the as-received interlayer metal. The variation of hardness values from the bulk values suggests that new phases formed because of the diffusion bonding process, and that these new phases at the interface have their own properties. In addition, the increase in hardness with respect to the as-received metals indicates that the new phases are more brittle. These brittle phases are likely to contribute to the cracks observed in the joints and to the degradation of the integrity of the joints. In addition, the microhardness results are supported by the results from EDS, XRD and SEM imaging, since they also showed the existence of newer phases. The new phases are identified as $\mathrm{Ni}, \mathrm{Cr}, \mathrm{Fe}$, and $\mathrm{Pd}$-silicides due to the interdiffusion of Si into the Inconel 600, and Ag into the SiC. These silicides are known to be brittle ceramic materials. These results are similar to those of G. Mahendran, et al. [48] who indicated that the increased in hardness with increasing time and temperature can be related to the formation of more intermetallic compounds (phases).

\section{6: Diffusion Kinetics}

The diffusion bonding behavior is influenced by elemental interdiffusion resulting in chemical reactions [3]. These chemical reactions form the reaction layers observed via 
the SEM imaging. Commonly, the thickness of these interfacial reaction layers are analyzed by their dependence to bonding time as represented in equation (5.5):

$$
\mathrm{x}=k t^{n}
$$

Where $\mathrm{k}$ is the reaction rate coefficient, $\mathrm{x}$ is the thickness measured, $\mathrm{t}$ is bonding time, and $\mathrm{n}$ is time exponent.

If the diffusion process is governed by the interfacial reaction, linear growth behavior is expected and $n=1$. If the diffusion process is governed by the volume diffusion, parabolic growth is expected and $n=1 / 2$ [77]. In this work, an assumption was made that $n=1 / 2$ and therefore equation 5.5 becomes:

$$
\mathrm{x}=k t^{1 / 2}
$$

When reorganized, in equation 5.6, the rate constant can be calculated by equation 5.7:

$$
k=x / t^{1 / 2}
$$

Assuming the process is thermally activated, it can be described by an Arrhenius relationship eq. 5.8. Accordingly, the activation energy of the rate limiting process can be found by reorganizing equation 5.8 into equation 5.9:

$$
\begin{aligned}
k & =k_{0} \exp \left(-\frac{Q}{R T}\right) \\
\ln k & =\ln k_{0}-\frac{Q}{R} \frac{1}{T}
\end{aligned}
$$


Where $k_{0}$ is the pre-exponential factor independent of temperature, $Q$ is the activation energy of the rate limiting process, $R=8.3145 \mathrm{fol} /(\mathrm{molK})$ is the ideal gas constant, and $T+(K)$ is bonding temperature.

Figure 48 shows the relationship between the thickness measured and the bonding times used (Table 14). The linear fit indicates that the reaction followed parabolic kinetics and therefore the process is diffusion-limited, also called diffusion-controlled process [78]. The reaction rate is related to the slope of the line in the thickness vs square root of bonding time plot (equation 5.7). The reaction rate is temperature dependent and follows an Arrhenius behavior, as described by equation 5.8 .

Focusing on the Ag interlayer system results, there are two outliers on the plot. These two data points indicate that something changed either in the process or in the material system used. The two data points that do not match the linear fit are attributed to a change in the $\mathrm{SiC}$ bulk material. If the concentration of free $\mathrm{Si}$ within this $\mathrm{SiC}$ matrix is larger, a larger diffusion thickness would be expected. The excess or free Si plays a role in the substitutional diffusion of $\mathrm{Ag}$ and $\mathrm{Si}$ across the interface. Parabolic kinetics is expected in solid state diffusion reactions, as demonstrated by W. M Tang, et al. [13], Ammar Khawam, and D. R. Flanagan [78], M. Jackson, et al. [62], P. He and D. Liu [79], and Jose Lemus-Ruiz [3]. The activation energy can be calculated if this process was repeated at different temperatures and the different reaction rates were plotted vs. the inverse of temperature. Equation 5.9 shows that the slope of the line in this case would be Q/R and represents a specific diffusion process. 


\section{CHAPTER SIX: FUTURE WORK}

Because of the complexity of these presented systems, further investigation is required to optimize the process parameters to fabricate the optimum Inconel 600/interlayer/SiC joint. This chapter presents what other variables and testing could be interesting to evaluate continue exploring the fabrication of this Inconel 600/interlayer/SiC material system for high temperature applications. The considerations are as follow:

1) Explore different purity of $\mathrm{SiC}$ materials to investigate in further detail the effect of free $\mathrm{Si}$ within the $\mathrm{SiC}$ matrix. From current investigations it was observed that it influences the diffusion process because it substitutes for the Ag material to diffuse into the Inconel 600, react and form silicide phases.

2) Since it was observed that $10 \mathrm{MPa}$ influence the yielding of the interlayer material and consequently also the diffusion process. The study of different pressure values can help to optimize this parameter to obtain premium intimate contact.

3) Similarly, to consideration two, it would be interesting to explore shorter times to evaluate how fast bonding can be achieved at specially if higher temperature and pressure is considered.

4) To further understand the impact of the interlayer component, different interlayer thickness can potentially be explored to minimize the loss of the interlayer component from the interface due to diffusion. 
5) To continue investigating the integrity of the fabricated assemblies, thermo-cycling testing under different operational temperatures is a valuable option to further understand the performance and reliability of the fabricated joints when experiencing changes in temperature.

6) Evaluate different geometries to study how geometry impacts stresses within the assembly that will translate in cracks and failure of the assemblies can also be an interesting approach, as well as if rectangle shape considered some shear testing can help to better quantify the bonding strength of the fabricated assemblies.

7) In order to expand the diffusion studies, multiple temperatures would have been explore to then as shown in Figure 53, different correlations between diffusion thickness and time using equation (5.6) would have been done. Multiple rate constants calculated using equation (5.7) or extracted from the fitted line and a better understanding of its temperature dependency would have also been understood. In addition, using equations (5.8 and 5.9) the activation energy of the effective diffusion bonding process investigate would have been extrapolated as shown in Figure 54. 


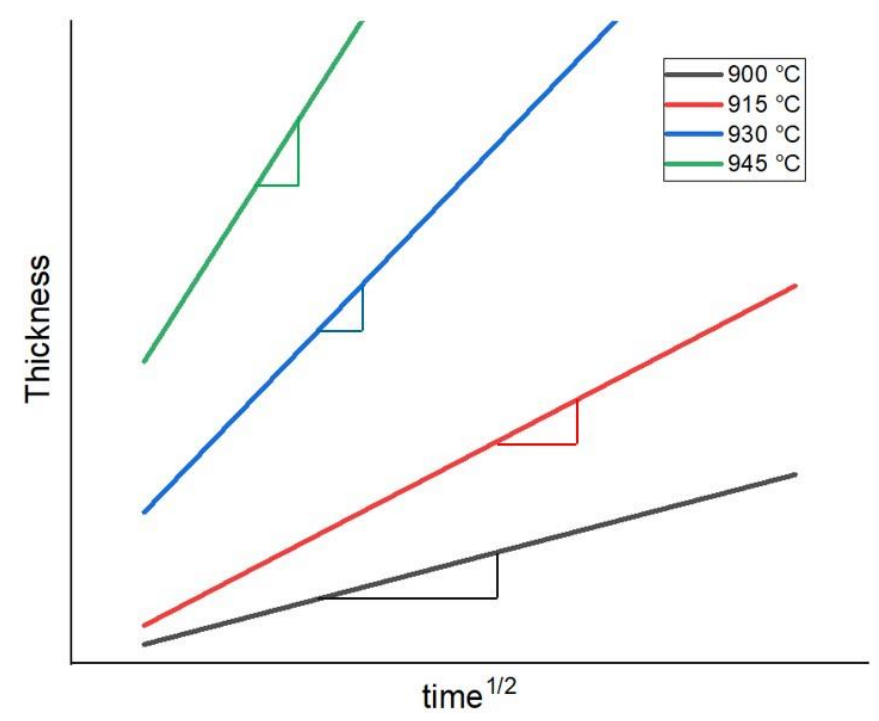

Figure 53 Plot of thickness vs square root of time of process performed at different temperatures to evaluate their correlation and extrapolate the rate constant that describes the progress of the process and understand how it depends on temperature. The rate constant is the slope of the fitted lines squared.

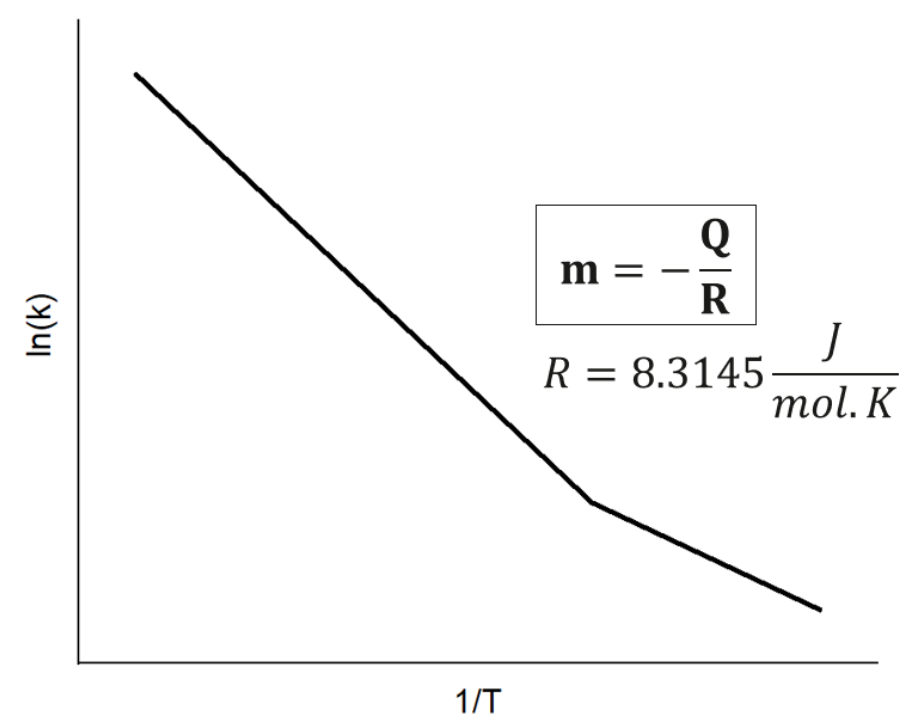

Figure $54 \quad$ Plot of natural $\log$ of $\mathrm{k}$ vs 1/T to evaluate the correlation and extrapolate the activation energy that the process required. The activation energy is the slope of the fitted lines squared.

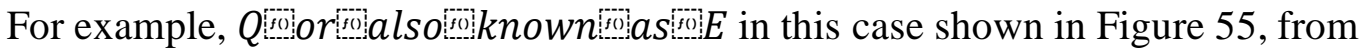
the work done by by Yajie Guo, et al. [77], can be found from plotting $\ln k$ vs $1 /$ T since $Q$ is the slope of the lines. And by comparing different material systems the dependency of 
that diffusion has on material systems can be evaluated, which could help with the material selection process.

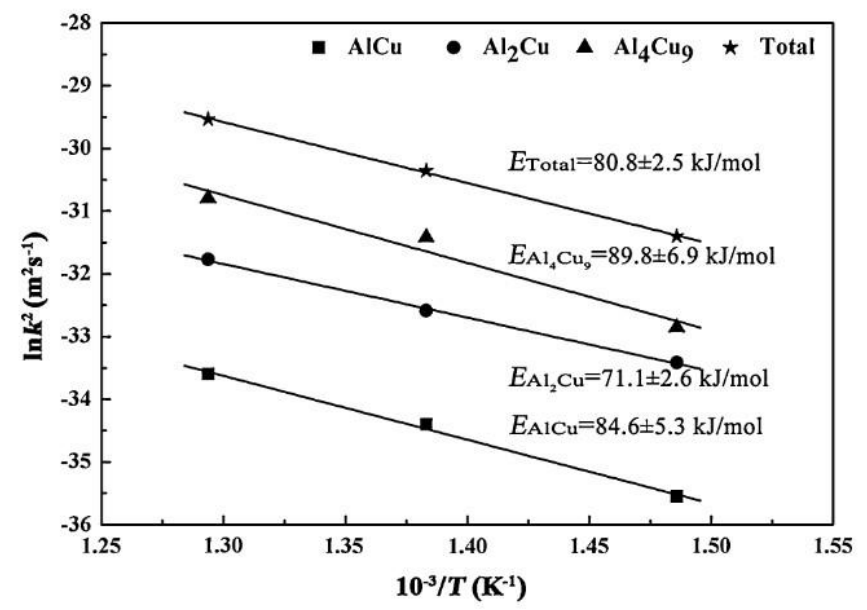

Figure 55 An Arrhenius plot of the $\mathrm{Al}_{4} \mathrm{Cu}$, $\mathrm{AlCu}, \mathrm{Al}_{2} \mathrm{Cu}$, and total intermetallic layer growth from [77]. 


\section{CHAPTER SEVEN: CONCLUSIONS}

This chapter presents the conclusions that have been drawn from several experiments done in this investigation. The formation of $\mathrm{SiC}$ to Inconel 600 joints using $\mathrm{Ag}$ and $\mathrm{AgPd}$ as interlayers has been shown and demonstrated using different diffusion bonding parameters, such as bonding temperature, applied pressure, and bonding time. Chemical reactions formed at the interface of the ceramic/metal joints have been analyzed by characterizing the cross-section of the fabricated Inconel/SiC transitions. The effect of the process parameters on the resultant interfacial microstructure has also been explained and the correlation between the interfacial solid-state reactions and the mechanical properties of the joints as well as the diffusion kinetics was described.

\section{1: Conclusions on the Inconel 600/Ag/SiC Joints}

From the results obtained about this material system, the following conclusions are presented:

1. The mass transfer of $\mathrm{Ag}$ into the $\mathrm{SiC}$ substituting the free Si that diffused into the Inconel 600 component was limited at $900{ }^{\circ} \mathrm{C}, 1 \mathrm{MPa}$ and 30 minutes when compared to other fabricated joints.

2. The temperature influenced the likelihood of formation of phases at the interface and promotes mass transfer due to the dependency of both on temperature because of thermodynamics.

3. Increasing the applied pressure increased the deformation of the interlayer and appeared to increase mass transfer across the interface. 
4. The main effect that holding time had was the impact on the thickness of the diffusion and reaction layers into the $\mathrm{SiC}$ component, as described by the diffusion kinetics evaluation.

5. The formation of silicide phases ( $\mathrm{Fe}, \mathrm{Ni}$, and $\mathrm{Cr}$ ) led to brittle regions and crack propagation along the metal/ceramic interface from large mismatch of CTE between components or reduction of a ductile, buffer layer to absorb the CTE mismatch.

6. The amount of free $\mathrm{Si}$ present in the $\mathrm{SiC}$ affected the diffusion of the $\mathrm{Ag}$ interlayer into the $\mathrm{SiC}$ component and the free Si into the Inconel 600 component leading to the formation of these phases.

7. The diffusion mechanism followed parabolic kinetics, which is indicative that the process is diffusion controlled.

\section{2: Conclusions on the Inconel 600/Ag75Pd25/SiC Joints}

From the results obtained about this material system, the following conclusions are presented:

1. Significant mass transfer was observed with any of the process parameter combinations used in this investigation.

2. The temperature influenced the likeliness of the formation of phases at the interface.

3. Increasing the applied pressure increased the diffusion of the interlayer into the SiC component.

4. Increasing holding time influenced the growth of the reaction layers. 
5. The formation of silicide phases ( $\mathrm{Fe}, \mathrm{Ni}, \mathrm{Cr}$, and $\mathrm{Pd})$ led to brittle regions and major cracking propagation along the metal/ceramic interface. Larger reaction thicknesses for all the process combinations was achieved in comparison to the Ag interlayer system.

6. The amount of Pd present in the interface catalyzed the diffusion of Ag into the SiC component.

7. The diffusion mechanism also followed parabolic kinetics, which is indicative that the process is diffusion controlled.

In summary, pressure was required to secure intimate contact. Once this intimate contact was achieved temperature and time governed the bonding process. Interlayer composition matters since it was concluded that the interlayer with Pd concentration had the diffusion process accelerated. This effect was observed when comparing the interfacial microstructure of two joints fabricated with the same process parameters but different interlayers and the interlayer with Pd concentration showed a much more complex interface. Finally, the formation of brittle intermetallic silicide phases at the interface result on a weak interface and compromised the reliability and integrity of the fabricated assemblies.

\section{REFERENCES}

[1] G. F. Blank, Garret, B. R. Randadive, A. J., "Broad applications of diffusion bonding," NASA, United States, Technical Report 1966.

[2] M. M. Schwartz, Ceramic Joining. Materials Park, Ohio: ASM INTERNATIONAL, 1990. 
[3] J. Lemus-Ruiz, "Diffusion Bonding of Silicon Nitride to Titanium," Doctor of Philosophy Traditional Manuscript, Department of Mining and Metallurgical Engineering, McGill University, Montreal, 0-612-70076-3, 2000.

[4] A. Passerone and M. L. Muolo, "Joining Technology in Metal-Ceramic Systems," Materials and Manufacturing Processes, vol. 15, no. 5, pp. 631-648, 2000/08/01 2000, doi: 10.1080/10426910008913010.

[5] G. Çam and M. Koçak, "Progress in joining of advanced materials," International Materials Reviews, vol. 43, no. 1, pp. 1-44, 1998/01/01 1998. [Online]. Available: https://doi.org/10.1179/imr.1998.43.1.1.

[6] C. Lewinsohn, M. Wilson, J. Fellows, and H. Anderson, "Fabrication and Joining of Ceramic Compact Heat Exchangers for Process Integration," International Journal of Applied Ceramic Technology, vol. 9, 07/01 2012.

[7] O. Akselsen, "Review of Diffusion Bonding of Ceramics," Journal of Materials Science, vol. 27, pp. 569-579, 02/01 1992, doi: 10.1007/BF02403862.

[8] M. L. Hattali, S. Valette, F. Ropital, G. Stremsdoerfer, N. Mesrati, and D. Tréheux, "Study of SiC-nickel alloy bonding for high temperature applications," J Eur Ceram Soc, vol. 29, no. 4, pp. 813-819, 2009/03/01/ 2009, doi: https://doi.org/10.1016/j.jeurceramsoc.2008.06.035.

[9] J. Li and P. Xiao, "Fabrication and characterisation of silicon carbide/superalloy interfaces," Journal of the European Ceramic Society, vol. 24, no. 7, pp. 21492156, 2004, doi: 10.1016/s0955-2219(03)00369-8.

[10] K. Bhanumurthy and R. Schmid-Fetzer, "Solid-state reaction bonding of silicon carbide (HIPSiC) below $1000^{\circ} \mathrm{C}, "$ Materials Science and Engineering: A, vol. 220, no. 1, pp. 35-40, 1996/12/15/ 1996, doi: https://doi.org/10.1016/S09215093(96)10452-4.

[11] L. Junqin, Z. Guangming, and X. Ping, "Joining reaction-bonded silicon carbide using Inconel 600 superalloy," J Mater Sci Lett, Article vol. 22, no. 10, pp. 759761, 2003, doi: 10.1023/A:1023768414427.

[12] J. R. McDermid, M. D. Pugh, and R. A. L. Drew, "The interaction of reactionbonded silicon carbide and Inconel 600 with a nickel-based brazing alloy," Metallurgical Transactions A, vol. 20, no. 9, pp. 1803-1810, 1989, doi: 10.1007/BF02663211. 
[13] W. M. Tang, Z. X. Zheng, H. F. Ding, and Z. H. Jin, "A study of the solid state reaction between silicon carbide and iron," Mater Chem Phys, vol. 74, no. 3, pp. 258-264, 2002/04/01/ 2002, doi: https://doi.org/10.1016/S0254-0584(01)00480-1.

[14] Z. Zhong, T. Hinoki, H.-C. Jung, Y.-H. Park, and A. Kohyama, "Microstructure and mechanical properties of diffusion bonded SiC/steel joint using W/Ni interlayer," Materials \& Design, vol. 31, no. 3, pp. 1070-1076, 2010/03/01/2010, doi: https://doi.org/10.1016/j.matdes.2009.09.049.

[15] Z. Zhong, T. Hinoki, and A. Kohyama, "Joining of Silicon Carbide to Ferritic Stainless Steel Using a W-Pd-Ni Interlayer for High-Temperature Applications," International Journal of Applied Ceramic Technology, vol. 7, no. 3, pp. 338-347, 2010/05/01 2010, doi: 10.1111/j.1744-7402.2009.02461.x.

[16] G. Elssner and G. Petzow, "Metal/Ceramic Joining," Isij Int, vol. 30, no. 12, pp. 1011-1032, 1990, doi: 10.2355/isijinternational.30.1011.

[17] S. P. Pandey and V. Singh, "The Importance of Engineering Materials in Present World," International Journal of Science and Research, 2015.

[18] William D. Callister Jr. and D. G. Rethwisch, Material Science and Engineering: An Introduction, 7th Edition ed. New York: John Wiley \& Sons, 2007.

[19] S. M. Allen and E. L. Thomas, The Structure of Materials. New York, Chichester, Weinheim, Brisbane, Singapore, Toronto: John Wiley \& Sons, Inc., 1999.

[20] Y. Zhang, D. Feng, Z.-y. He, and X.-c. Chen, "Progress in Joining Ceramics to Metals," Journal of Iron and Steel Research, International, vol. 13, no. 2, pp. 1-5, 2006/03/01/ 2006, doi: https://doi.org/10.1016/S1006-706X(06)60032-0.

[21] Uday M.B, Ahmad-Fauzi M.N, Alias Mohd Noor, and S. Rajoo, "Current Issues and Problems in the Joining of Ceramic to Metal," in Joining Technologies, M. Ishak Ed., 2016, p. 35.

[22] A. Tomsia, "Ceramic/metal joining for structures and materials," (in English), Journal de Physique IV Colloque, vol. 03, no. C7, pp. C7-1317-C7-1326, 1993 1993, doi: 10.1051/jp4:19937203.

[23] J. Lindroos et al., "Nickel: A very fast diffuser in silicon," Journal of Applied Physics, vol. 113, no. 20, p. 204906, 2013, doi: 10.1063/1.4807799. 
[24] T. Isobe, H. Nakashima, and K. Hashimoto, "Diffusion Coefficient of Interstitial Iron in Silicon," Japanese Journal of Applied Physics, vol. 28, no. Part 1, No. 7, pp. 1282-1283, 1989/07/20 1989, doi: 10.1143/jjap.28.1282.

[25] A. K. Jadoon, B. Ralph, and P. R. Hornsby, "Metal to ceramic joining via a metallic interlayer bonding technique," J Mater Process Tech, vol. 152, no. 3, pp. 257-265, 2004/10/30/ 2004, doi: https://doi.org/10.1016/j.jmatprotec.2003.10.005.

[26] CES EduPack software

[27] F. C. Nix and D. MacNair, "The Thermal Expansion of Pure Metals: Copper, Gold, Aluminum, Nickel, and Iron," Physical Review, vol. 60, no. 8, pp. 597-605, 10/15/ 1941, doi: 10.1103/PhysRev.60.597.

[28] S. Metals, "Inconel 600 Data Sheet," www.specialmetals.com.

[29] S. M. Corporation. Inconel alloy 600

[30] CoorsTek. "Reaction Bonded Silicon Carbide." (accessed 2020).

[31] J. Martínez Fernández, A. Muñoz, A. R. de Arellano López, F. M. Valera Feria, A. Domínguez-Rodríguez, and M. Singh, "Microstructure-mechanical properties correlation in siliconized silicon carbide ceramics," Acta Mater, vol. 51, no. 11, pp. 3259-3275, 2003/06/27/ 2003, doi:

https://doi.org/10.1016/S13596454(03)00157-5.

[32] D. Kopeliovich. "Fabrication of Ceramic Matrix Composites by Liquid Silicon Infiltration (LSI)." SubsTech Substances \& Technologies. (accessed 2020).

[33] M. Singh and E. Lara-Curzio, "Design, fabrication, and testing of ceramic joints for high temperature SiC/SiC composites," (in English), J Eng Gas Turb Power, vol. 123, no. 2, pp. 288-292, Apr 2001, doi: Doi 10.1115/1.1362664.

[34] RioGrande. ".999 Fine Silver Strip, Dead Soft." (accessed 2020).

[35] E. Metals. "Silver-Pd25\% Sheet .010"." (accessed 2020).

[36] B. S. Univeristy. "Boise State Center for Materials Characterization, Instrumentation." https://www.boisestate.edu/coen-bscmc/instrumentation/ (accessed 2020).

[37] H. Group. "Scanning Electron Microscope S-3700N." Hitachi High Technology in America. https://www.hitachi-hightech.com/us/product_detail/?pn=em-s3700n (accessed 2020). 
[38] Rigaku. "Benchtop Poweder X-ray Diffraction (XRD) Miniflex Instrument." Rigaku Corporation. https://www.rigaku.com/products/xrd/miniflex (accessed 2020).

[39] Ravindra Kumar, Yogesh Chandra Sharma, Vijja Vidya Sagar, and D. Bhardwaj, "Characterization of low temperature plasma ion nitriding (Pin) of Inconel 600 alloys," p. 16.

[40] W. Liu, N. Yan, and H. Wang, "Dendritic morphology evolution and microhardness enhancement of rapidly solidified Ni-based superalloys," Science China Technological Sciences, vol. 62, no. 11, pp. 1976-1986, 2019/11/01 2019, doi: 10.1007/s11431-019-9519-9.

[41] J. Schindelin et al., "Fiji: an open-source platform for biological-image analysis," Nat Methods, vol. 9, no. 7, pp. 676-682, 2012/07/01 2012, doi: 10.1038/nmeth.2019.

[42] C. T. Rueden et al., "ImageJ2: ImageJ for the next generation of scientific image data," BMC Bioinformatics, vol. 18, no. 1, p. 529, 2017/11/29 2017, doi: 10.1186/s12859-017-1934-Z.

[43] M. E. Schlesinger, "Thermodynamics of solid transition-metal silicides," Chemical Reviews, vol. 90, no. 4, pp. 607-628, 1990/06/01 1990, doi: $10.1021 / \mathrm{cr} 00102 \mathrm{a} 003$.

[44] W. Tang, B.-M. Nguyen, R. Chen, and S. A. Dayeh, "Solid-state reaction of nickel silicide and germanide contacts to semiconductor nanochannels," Semiconductor Science and Technology, vol. 29, no. 5, p. 054004, 2014/04/01 2014, doi: 10.1088/0268-1242/29/5/054004.

[45] I. Barin, Thermochemical data of pure substances, Third edition. ed. Weinheim ;: VCH, 1995.

[46] R. Pretorius, "Prediction of silicide formation and stability using heats of formation," Thin Solid Films, vol. 290-291, pp. 477-484, 1996/12/15/ 1996, doi: https://doi.org/10.1016/S0040-6090(96)09022-0.

[47] R. O'Hayre, Materials Kinetics Fundamentals. Hoboken, New Jersey: John Wiley \& Sons, Inc., 2015. 
[48] G. Mahendran, V. Balasubramanian, and T. Senthilvelan, "Influences of diffusion bonding process parameters on bond characteristics of $\mathrm{Mg}-\mathrm{Cu}$ dissimilar joints," $T$ Nonferr Metal Soc, vol. 20, no. 6, pp. 997-1005, 2010/06/01/ 2010, doi: https://doi.org/10.1016/S1003-6326(09)60248-X.

[49] T. Gietzelt, V. Toth, and A. Huell, "Diffusion Bonding: Influence of Process Parameters and Material Microstructure," 2016.

[50] M. Samavatian, S. Zakipour, and M. Paidar, "Effect of bonding pressure on microstructure and mechanical properties of Ti-6Al-4V diffusion-bonded joint," Welding in the World, vol. 61, 10/19 2016, doi: 10.1007/s40194-016-0390-0.

[51] D. Aboudi, S. Lebaili, M. Taouinet, and J. Zollinger, "Microstructure evolution of diffusion welded 304L/Zircaloy4 with copper interlayer," Mater Design, vol. 116, pp. 386-394, 2017/02/15/ 2017, doi:

https://doi.org/10.1016/i.matdes.2016.12.008.

[52] J. H. Neethling, J. H. O’Connell, and E. J. Olivier, "Palladium assisted silver transport in polycrystalline SiC," Nuclear Engineering and Design, vol. 251, pp. 230-234, 2012/10/01/ 2012, doi: https://doi.org/10.1016/j.nucengdes.2011.09.064.

[53] E. J. Olivier and J. H. Neethling, "The role of Pd in the transport of Ag in SiC," J Nucl Mater, vol. 432, no. 1-3, pp. 252-260, 2013, doi:

10.1016/j.jnucmat.2012.07.033.

[54] R. L. Pearson, R. J. Lauf, and T. B. Lindemer, "Interaction of palladium, the rare earths, and silver with silicon carbide in HTGR fuel particles," United States, 1982-04-01 1982. [Online]. Available:

https://www.osti.gov/servlets/purl/5341304

[55] M. Gentile, P. Xiao, and T. Abram, "Palladium interaction with silicon carbide," Journal of Nuclear Materials, vol. 462, pp. 100-107, 2015/07/01/ 2015, doi: https://doi.org/10.1016/j.jnucmat.2015.03.013.

[56] K. Bhanumurthy and R. Schmid-Fetzer, "Interface reactions between silicon carbide and metals (Ni, Cr, Pd, Zr)," Composites Part A: Applied Science and Manufacturing, vol. 32, no. 3, pp. 569-574, 2001/03/01/ 2001, doi: https://doi.org/10.1016/S1359-835X(00)00049-X.

[57] M. Backhaus- Ricoult, Solid State Reactions Between Silicon Carbide and Various Transition Metals. 1989, pp. 1277-1281. 
[58] H. J. MacLean, R. G. Ballinger, L. E. Kolaya, S. A. Simonson, N. Lewis, and M. E. Hanson, "The effect of annealing at $1500^{\circ} \mathrm{C}$ on migration and release of ion implanted silver in CVD silicon carbide," J Nucl Mater, vol. 357, no. 1, pp. 3147, 2006/10/15/ 2006, doi: https://doi.org/10.1016/j.jnucmat.2006.05.043.

[59] E. Friedland et al., "Study of silver diffusion in silicon carbide," Journal of Nuclear Materials, vol. 389, no. 2, pp. 326-331, 2009/05/31/ 2009, doi: https://doi.org/10.1016/j.jnucmat.2009.02.022.

[60] P. Villars, H. Ohamoto, and K. Cenzual. ASM Alloy Phase Diagram database

[61] M. Backhaus-Ricoult, "Solid state reactions between silicon carbide and ( $\mathrm{Fe}, \mathrm{Ni}$, Cr)-alloys: reaction paths, kinetics and morphology," Acta Metall Mater, vol. 40, pp. S95-S103, 1992. [Online]. Available: http://www.sciencedirect.com/science/article/pii/095671519290268J.

[62] M. R. Jackson, R. L. Mehan, A. M. Davis, and E. L. Hall, "Solid state SiC/Ni alloy reaction," Metallurgical Transactions A, vol. 14, no. 2, pp. 355-364, 1983/02/01 1983, doi: 10.1007/BF02644213.

[63] F. M. d'Heurle and P. Gas, "Kinetics of formation of silicides: A review," Journal of Materials Research, vol. 1, no. 1, pp. 205-221, 1986, doi: 10.1557/JMR.1986.0205.

[64] L. A. Clevenger, C. V. Thompson, R. C. Cammarata, and K. N. Tu, "Reaction kinetics of nickel/silicon multilayer films," Applied Physics Letters, vol. 52, no. 10, pp. 795-797, 1988/03/07 1988, doi: 10.1063/1.99644.

[65] Christian Lavoie et al., Reactive Diffusion in the Ni-Si System: Phase Sequence and Formation of Metal-Rich Phases. 2005, pp. 825-836.

[66] F. J. J. van Loo, M. R. Rijnders, K. J. Rönkä, J. H. Gülpen, and A. A. Kodentsov, "Solid state diffusion and reactive phase formation," Solid State Ionics, vol. 95, no. 1, pp. 95-106, 1997/02/02/ 1997, doi: https://doi.org/10.1016/S01672738(96)00550-4.

[67] R. C. J. Schiepers, J. A. van Beek, F. J. J. van Loo, and G. de With, "The interaction between $\mathrm{SiC}$ and $\mathrm{Ni}, \mathrm{Fe},(\mathrm{Fe}, \mathrm{Ni})$ and steel: Morphology and kinetics," J Eur Ceram Soc, vol. 11, no. 3, pp. 211-218, 1993/01/01/ 1993, doi: https://doi.org/10.1016/0955-2219(93)90090-E. 
[68] D. Connétable and O. Thomas, "First-principles study of nickel-silicides ordered phases," J Alloy Compd, vol. 509, no. 6, pp. 2639-2644, 2011/02/10/ 2011, doi: https://doi.org/10.1016/j.jallcom.2010.10.118.

[69] F. Nemouchi, D. Mangelinck, C. Bergman, P. Gas, and U. Smith, "Differential scanning calorimetry analysis of the linear parabolic growth of nanometric $\mathrm{Ni}$ silicide thin films on a Si substrate," Applied Physics Letters, vol. 86, no. 4, p. 041903, 2005/01/24 2005, doi: 10.1063/1.1852727.

[70] J. William D. Callister and D. G. Rethwisch, Material Science and Engineering: An Introduction, 9th Edition ed. New York: John Wiley \& Sons, 2014.

[71] J. Krenos, "Chemical Kinetics and Reaction Dynamics (by Paul L. Houston)," Journal of Chemical Education - J CHEM EDUC, vol. 78, 11/01 2001, doi: 10.1021/ed078p1466.

[72] Z. Wen, Y. Zhao, H. Hou, and L. Chen, "First-Principles Investigation of Mechanical and Thermodynamic Properties of Nickel Silicides at Finite Temperature," Physics of the Solid State, vol. 60, no. 5, pp. 967-974, 2018/05/01 2018, doi: 10.1134/S1063783418050360.

[73] J. C. Feng, H. J. Liu, M. Naka, and J. C. Schuster, "Reaction products and growth kinetics during diffusion bonding of $\mathrm{SiC}$ ceramic to $\mathrm{Ni}-\mathrm{Cr}$ alloy," Mater Sci Tech Ser, vol. 19, no. 1, pp. 137-142, 2003.

[74] J. S. Park, K. Landry, and J. H. Perepezko, "Kinetic control of silicon carbide/metal reactions," Materials Science and Engineering: A, vol. 259, no. 2, pp. 279-286, 1999/01/31/ 1999, doi: https://doi.org/10.1016/S09215093(98)00899$\underline{5}$.

[75] M. R. A. Evans, M. Turwitt, "ON THE MECHANICS OF FAILURE IN CERAMIC/METAL BONDED SYSTEMS," Journal de Physique Colloques, vol. 46 (C4), pp. pp.C4-613-C4-626, 1985.

[76] C. Zhang, H. Li, and M. Li, "Detailed Evolution Mechanism of Interfacial Void Morphology in Diffusion Bonding," Journal of Materials Science \& Technology, vol. 32, no. 3, pp. 259-264, 2016/03/01/ 2016, doi: https://doi.org/10.1016/j.jmst.2015.12.002. 
[77] Y. Guo, G. Liu, H. Jin, Z. Shi, and G. Qiao, "Intermetallic phase formation in diffusion-bonded Cu/Al laminates," J Mater Sci, vol. 46, no. 8, pp. 2467-2473, 2011/04/01 2011, doi: 10.1007/s10853-010-5093-0.

[78] A. Khawam and D. R. Flanagan, "Solid-State Kinetic Models: Basics and Mathematical Fundamentals," The Journal of Physical Chemistry B, vol. 110, no. 35, pp. 17315-17328, 2006/09/01 2006, doi: 10.1021/jp062746a.

[79] P. He and D. Liu, "Mechanism of forming interfacial intermetallic compounds at interface for solid state diffusion bonding of dissimilar materials," Materials Science and Engineering: A, vol. 437, no. 2, pp. 430-435, 2006/11/15/ 2006, doi: https://doi.org/10.1016/j.msea.2006.08.019. 